Pacific

Journal of

Mathematics

CR YAMABE CONJECTURE - THE CONFORMALLY FLAT CASE

Najoua Gamara and Ridha Yacoub

Volume $201 \quad$ No. 1

November 2001 


\title{
CR YAMABE CONJECTURE - THE CONFORMALLY FLAT CASE
}

\author{
Najoua Gamara AND Ridha YaCOUB
}

We extend the resolution of the CR Yamabe conjecture for one of the two cases left open by D. Jerison and J.M. Lee: The CR locally conformally flat case.

The proof is based on techniques related to the theory of critical points at infinity. These techniques were first introduced by A. Bahri, A. Bahri and $\mathrm{H}$. Brezis for the resolution of the same conjecture for the Riemannian case.

\section{Introduction.}

Let $(M, \theta)$ be an orientable compact real $(2 n+1)$-dimensional CR manifold, with a given contact form $\theta$. Denote by $L=L_{\theta}=\left(2+\frac{2}{n}\right) \Delta_{b}+R_{\theta}$ the CR conformal laplacian on $M$, where $\Delta_{b}$ is the sublaplacian operator (see Section 1) and $R_{\theta}$ the Webster scalar curvature associated to $\theta$.

The CR Yamabe Conjecture states that there exists a contact form $\widetilde{\theta}$ on $M$, CR equivalent to $\theta$, with constant Webster scalar curvature $\widetilde{R}_{\widetilde{\theta}}$. This conjecture is equivalent to the existence of a function $u$ such that:

$$
\left\{\begin{array}{l}
L u=u^{1+\frac{2}{n}} \\
u>0 \text { on } M
\end{array}\right.
$$

(1) is a particular case of more general equations of the type:

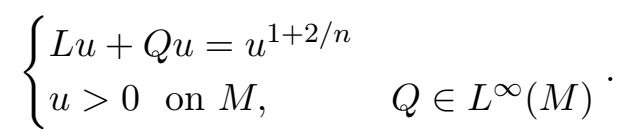

D. Jerison and J.M. Lee have extensively studied the CR Yamabe conjecture $([\mathbf{1 0}],[\mathbf{1 1}]$ and $[\mathbf{1 2}])$. They showed that there was a deep analogy between the CR Yamabe problem, and the Riemannian Yamabe conjecture which was solved by T. Aubin [1] and R. Schoen [18].

T. Aubin [1] proved the conjecture in the two following cases:

1) $(N, g)$ is a not conformally flat compact Riemannian manifold of dimension $n \geq 6$.

2) $(N, g)$ is a locally conformally flat compact Riemannian manifold, with dimension $n \geq 3$ and finite Poincaré group, not conformal to $\left(S^{n}, c\right)$, where $S^{n}$ is the $n$-dimensional sphere with its standard metric $c$. 
R. Schoen [18] solved the Yamabe problem in the remaining cases, using the positive mass theorem.

In the CR case, D. Jerison and J.M. Lee, gave a necessary condition on the CR invariant:

$$
\lambda(M)=\inf _{u \in S_{1}^{2}(M)}\left\{A_{\theta}(u) \mid B_{\theta}(u)=1\right\}
$$

to solve Equation (1).

Here, $S_{1}^{2}(M)$ is a Folland and Stein space (see Section 4),

$$
A_{\theta}(u)=\int_{M}\left(\left(2+\frac{2}{n}\right)|d u|_{\theta}^{2}+R_{\theta} u^{2}\right) \theta \wedge d \theta^{n}
$$

the functional associated to the CR Yamabe equation, where $|d u|_{\theta}$ is the norm of the cotangent vector $d u$ (see [10] or Section 1 for its precise formula) and $B_{\theta}(u)=\int_{M}|u|^{2+\frac{2}{n}} \theta \wedge d \theta^{n}$.

This condition is summarized by the following result:

Theorem $([\mathbf{1 0}, 3.4]$.$) . Let M$ be an orientable compact $(2 n+1)$-dimensional CR manifold, $\theta$ any contact form on $M$.

(a) $\lambda(M)$ depends only on the CR structure of $M$, not of the choice of $\theta$.

(b) $\lambda(M) \leq \lambda\left(S^{2 n+1}\right)$, where $S^{2 n+1} \subset \mathbb{C}^{n+1}$ is the sphere with its standard CR structure.

(c) If $\lambda(M)<\lambda\left(S^{2 n+1}\right)$, then (1) has a solution.

Furthermore, D. Jerison and J.M. Lee proved the CR Yamabe conjecture for $\mathrm{CR}$ manifolds which are not locally $\mathrm{CR}$ equivalent to the sphere of the same dimension:

Theorem ([11, A]). Suppose $M$ is a compact strictly pseudoconvex $(2 n+1)$ dimensional $\mathrm{CR}$ manifold. If $n \geq 2$ and $M$ is not locally $\mathrm{CR}$ equivalent to $S^{2 n+1}$, then $\lambda(M)<\lambda\left(S^{2 n+1}\right)$, and thus, the CR Yamabe problem can be solved on $M$.

The results of D. Jerison and J.M. Lee can be formally compared to the partial completion of the proof of the Riemannian Yamabe conjecture by T. Aubin [1]. The remaining cases should, by analogy, be solved using some CR positive mass theorem. Unfortunately, especially because the theory of CR minimal surfaces does not exist at the present time, such a CR version of the positive mass theorem is not available.

Besides the proof by T. Aubin and R. Schoen of the Riemannian Yamabe conjecture, another proof by A. Bahri [2], A. Bahri-H. Brezis [4] of the same conjecture is available by techniques related to the theory of critical points at infinity. This proof is completely different in spirit as well as in techniques and details, from T. Aubin and R. Schoen proof. It does not require the use of any theory of minimal surfaces, neither does it require the use of the 
positive mass theorem. It turns out that this proof can be carried out to the CR Yamabe conjecture as well as in [4].

We will not try here to give to this method its upmost generality in the CR context. We will be satisfied with the proof of the CR Yamabe conjecture in the cases left open by D. Jerison and J.M. Lee.

More precisely, we will focus in the present paper on the CR locally conformally flat case.

In [9], the case $\mathrm{n}=1$, but without any hypothesis of $\mathrm{CR}$ conformal flatness is studied.

We establish the following theorem:

Theorem 1. Let $(M, \theta)$ be an orientable compact real $(2 n+1)$-dimensional CR manifold, locally CR equivalent to $S^{2 n+1}$, then (1) has a solution.

The proof of Theorem 1 is based on a contradiction argument and involves several steps which will be detailed over six sections:

Let $H$ be the subspace of $S_{1}^{2}(M)$ defined by

$$
\begin{aligned}
& H=\left\{u \in S_{1}^{2}(M) \text { such that } \int_{M}|d u|_{\theta}^{2} \theta\right. \wedge d \theta^{n}<\infty \\
&\text { and } \left.\int_{M}|u|^{2+\frac{2}{n}} \theta \wedge d \theta^{n}<\infty\right\}
\end{aligned}
$$

let

where

$$
\sum=\left\{u \in H, \text { such that }\|u\|_{H}=1\right\}
$$

$$
\|u\|_{H}=\left(\int_{M}\left(\left(2+\frac{2}{n}\right)|d u|_{\theta}^{2}+R_{\theta} u^{2}\right) \theta \wedge d \theta^{n}\right)^{\frac{1}{2}}
$$

and let

$$
\sum_{+}=\left\{u \in \sum \mid u \geq 0\right\}
$$

For $u \in H$, we define:

$$
J(u)=\frac{\left(\int_{M}\left(\left(2+\frac{2}{n}\right)|d u|_{\theta}^{2}+R_{\theta} u^{2}\right) \theta \wedge d \theta^{n}\right)^{1+\frac{1}{n}}}{\int_{M}|u|^{2+\frac{2}{n}} \theta \wedge d \theta^{n}} .
$$

In the first section, we recall the general definition of CR manifolds, the CR Yamabe equation and the Yamabe functional $J$.

In Section 2, we recall the standard solutions of the CR Yamabe equation for the Heisenberg group $\mathbb{H}^{n}$. These solutions are obtained by left translations and dilations $(z, t) \mapsto\left(\lambda z, \lambda^{2} t\right),(\lambda \in \mathbb{R})$, on the Heisenberg group, of the function $u(z, t)=K|w+i|^{-n}$, where $w=t+i|z|^{2}$, and $K \in \mathbb{C}$.

Since the injection $S_{1}^{2}\left(\mathbb{H}^{n}\right) \longrightarrow L^{2+\frac{2}{n}}\left(\mathbb{H}^{n}\right)$ is continuous but not compact, the functional $J$ does not satisfy the Palais-Smale condition (denoted 
by (P.S)). More precisely, one can see that the standard solutions on $\mathbb{H}^{n}$, $u(z, t)=K|w+i|^{-n}$, after superposition are the good candidate sequences which violate the (P.S) condition. Therefore, the classical variational theory based on compactness arguments, does not apply in our case.

In Section 3, we study the case of a general CR manifold, locally CR equivalent to the sphere of the same dimension. We show the existence of a conformal factor $\widetilde{u}_{a}^{\frac{2}{n}}$ depending differentiably on $a \in M$, such that if the contact form $\theta$ is replaced by $\widetilde{u}_{a}^{\frac{2}{n}} \theta$ in a ball $B(a, \rho)$, then $\left(M, \widetilde{u}_{a}^{\frac{2}{n}} \theta\right)$ is locally $\left(\mathbb{H}^{n}, \theta_{0}\right)$, where $\theta_{0}$ is the standard contact form on $\mathbb{H}^{n}$. Therefore, we locally may use the usual multiplication of $\mathbb{H}^{n}$, we also may use in $B(a, \rho)$ the standard solutions of the CR Yamabe problem, which we denote by $\delta(b, \lambda)$, where $\lambda \in \mathbb{R}$. The function $\delta(b, \lambda)$ satisfies the following equation:

$$
\left\{\begin{array}{l}
L_{\theta_{0}} \delta(b, \lambda)=\delta(b, \lambda)^{1+\frac{2}{n}} \quad \text { on } B(a, \rho) \\
b \in B(a, \rho) .
\end{array}\right.
$$

We then define a family of "almost solutions" $\hat{\delta}(a, \lambda)$, to be the unique solutions of:

$$
L \hat{\delta}(a, \lambda)=\delta^{\prime}(a, \lambda)^{1+\frac{2}{n}} \text { on } M
$$

where

$$
\left\{\begin{array}{lll}
\delta^{\prime}(a, \lambda)=\omega_{a} \widetilde{u}_{a} \delta(a, \lambda) & \text { on } & B(a, \rho) \\
\delta^{\prime}(a, \lambda)=0 & \text { on } & \mathcal{C}_{B}(a, \rho)
\end{array}\right.
$$

$\omega_{a}$ is a cut-off function used to localize our function near the point $a$, when $\lambda \rightarrow+\infty$. And we show that:

$$
\left|\hat{\delta}-\delta^{\prime}\right|=O\left(\frac{1}{\lambda^{n}}\right)
$$

and

$$
\left|\hat{\delta}-\delta^{\prime}\right|_{\mathcal{C}^{2}}=O\left(\frac{1}{\lambda^{n}}\right)
$$

when $\lambda \rightarrow+\infty$.

Because of these estimates, we can replace, in the analysis of the (P.S) condition the functions $\delta^{\prime}$ or $\delta$ by the function $\hat{\delta}$.

In Section 4, we will characterize the sequences which violate the (P.S) condition. 
Let $V(p, \epsilon)$, for $\epsilon>0$ and $p \in \mathbb{N}^{*}$, be defined in analogy with the Riemannian case $([\mathbf{3}])$ by:

$$
V(p, \epsilon)=\left\{\begin{array}{c}
u \in \sum_{+} \text {such that there exists } p \text { concentration points } \\
a_{1}, \ldots, a_{p} \text { in } M \text { and } p \text { concentrations } \lambda_{1}, \ldots, \lambda_{p}>0 \\
\text { such that }\left\|u-\frac{1}{p^{\frac{1}{2}} S^{\frac{n}{2}}} \sum_{i=1}^{i=p} \hat{\delta}\left(a_{i}, \lambda_{i}\right)\right\|_{H}<\epsilon, \text { with } \lambda_{i} \geq \frac{1}{\epsilon} \\
\text { and } \forall i \neq j, \epsilon_{i j}=\frac{\lambda_{i}}{\lambda_{j}}+\frac{\lambda_{j}}{\lambda_{i}}+\lambda_{i} \lambda_{j} \widetilde{d}\left(a_{i}, a_{j}\right)^{2} \geq \frac{1}{\epsilon}
\end{array}\right\}
$$

where $\widetilde{d}(x, y)$, if $x$ and $y$ are in a small ball of $M$ of radius $\rho$, is $\left\|\exp _{x}^{-1}(y)\right\|_{\mathbb{H}^{n}}$ $\left(\|\cdot\|_{\mathbb{H}^{n}}\right.$ is the norm in $\left.\mathbb{H}^{n}\right)$, with $\exp _{x}$ the CR exponential map for the point $x$, and $\widetilde{d}(x, y)$ is equal to $\frac{\rho}{2}$ otherwise. $S$ is the Sobolev constant introduced in (5.1).

The set $V(p, \epsilon)$ has a simple interpretation: It is a neighborhood of the critical points at infinity of the functional $J$ on $\sum_{+}$.

Let $\left(u_{k}\right)$ be a sequence of $H$ satisfying $J^{\prime}\left(u_{k}\right) \rightarrow 0$, and $J\left(u_{k}\right)$ bounded. Then $\left(u_{k}\right)$ is a bounded sequence in $H$, hence $\left(u_{k}\right)$ has a weak limit $\bar{u}$.

If $\bar{u}$ is non-zero, we prove that $\bar{u}$ is a critical point of $J$ (Proposition 5).

Since we are going to prove Theorem 1 by contradiction, we assume that the weak limit $\bar{u}$, of any sequence $\left(u_{k}\right)$ of $H$ satisfying $J^{\prime}\left(u_{k}\right) \rightarrow 0$ and $J\left(u_{k}\right)$ bounded, is zero.

Then, assuming that $\left(u_{k}\right)$ is non-negative, we prove that we can extract from $\left(u_{k}\right)$ a subsequence denoted again by $\left(u_{k}\right)$, such that $\frac{u_{k}}{\left\|u_{k}\right\|_{H}} \in V\left(p, \epsilon_{k}\right)$ with $\epsilon_{k}>0$ and $\lim _{k \rightarrow+\infty} \epsilon_{k}=0$ (Proposition 8).

While the final characterization of the sequences which violate the (P.S) condition is basically identical to the Riemannian case, the proof of this fact is different here because no use of $H_{0}^{1}$-spaces can be made, with our current knowledge in the CR framework. M. Struwe [19] used such spaces and the projections on them in order to give a characterization of the sequences violating the (P.S) condition in the Riemannian framework.

In Section 5, we expand the functional $J$, near the set of potential critical points at infinity $V(p, \epsilon)$. We prove that for $p \geq p_{0}$ (i.e., for $p$ large enough) the energy $J\left(\sum_{i=1}^{i=p} \alpha_{i} \hat{\delta}\left(a_{i}, \lambda\right)\right)$ is less than the critical level at infinity for $p$ masses concentrated at points $a_{1}, \ldots, a_{p}$, i.e., $J\left(\sum_{i=1}^{i=p} \alpha_{i} \hat{\delta}\left(a_{i}, \lambda\right)\right) \leq p^{\frac{1}{n}} S$ for $\lambda \geq \lambda_{0}>0$. The $\alpha_{i}$ 's are positive and satisfy $\sum_{i=1}^{i=p} \alpha_{i}=1$. We now are ready for the final argument.

Considering for $p \in \mathbb{N}$ the barycentric sets:

$$
\begin{gathered}
B_{0}(M)=\emptyset \\
B_{p}(M)=\left\{\sum_{i=1}^{i=p} \alpha_{i} \delta_{x_{i}} \mid \sum_{i=1}^{i=p} \alpha_{i}=1, \alpha_{i} \geq 0, x_{i} \in M\right\}
\end{gathered}
$$


with $\delta_{x_{i}}$ the Dirac mass at $x_{i}$, and considering the level sets for the functional $J$ :

$$
W_{p}=\left\{u \in \sum_{+} \text {such that } J(u)<(p+1)^{\frac{1}{n}} S\right\}
$$

we define a map $f_{p}(\lambda)$ from $B_{p}(M)$ to $\sum_{+}$by the formula:

$$
f_{p}(\lambda)\left(\sum_{i=1}^{i=p} \alpha_{i} \delta_{x_{i}}\right)=\frac{\sum_{i=1}^{i=p} \alpha_{i} \hat{\delta}\left(x_{i}, \lambda\right)}{\left\|\sum_{i=1}^{i=p} \alpha_{i} \hat{\delta}\left(x_{i}, \lambda\right)\right\|_{H}} .
$$

Then, Section 5 implies that the map $f_{p}(\lambda)$ is homologically trivial for $p \geq p_{0}$ (Proposition 20).

On the other hand, assuming that problem (1) has no solution, we prove in Section 6 , by using topological arguments, that $f_{p *}(\lambda) \not \equiv 0$, for all $p \in \mathbb{N}$ (Proposition 22). This gives a contradiction to Proposition 20, and hence completes the proof of Theorem 1 .

The topological argument which we use has been first displayed in [5]. It is an intricate argument which we have somewhat made easier, for the convenience of an interested reader, in a simple case, in Appendix $\mathrm{C}$ of this paper.

\section{CR manifolds and CR Yamabe equation.}

Sections 1 and 2 are mainly extracted from the following references: D. Jerison and J.M. Lee [10], G.B. Folland and E.M. Stein [8]. They have been written for the sake of completeness of this paper.

Let $M$ be an orientable, real, $(2 n+1)$-dimensional manifold. A CR structure on $M$ is given by a complex $n$-dimensional subbundle $T_{1,0}$ of the complexified tangent bundle $\mathbb{C} T M$ of $M$, satisfying $T_{1,0} \cap T_{0,1}=\{0\}$, where $T_{0,1}=\bar{T}_{1,0}$. We assume that the Frobenius condition $\left[T_{1,0}, T_{1,0}\right] \subset T_{1,0}$ is satisfied.

We set $G=\mathcal{R e}\left(T_{1,0}+T_{0,1}\right)$ so that $G$ is a real $2 n$-dimensional subbundle of $T M$. $G$ carries a natural complex structure map $\jmath: G \longrightarrow G$, given by $\jmath(V+\bar{V})=i(V-\bar{V})$ for $V \in T_{1,0}$.

Let $E \subset T^{*} M$ denote the real line bundle $G^{\perp}$. Because we assume $M$ orientable, and, $G$ is oriented by its complex structure, $E$ has a global nonvanishing section $\theta$, which is called a contact form. To such a section is associated a real symmetric bilinear form $\ell_{\theta}$ on $G$, called the Levy form of $\theta$, and defined by:

$$
\ell_{\theta}(V, W)=2\langle d \theta, V \wedge \jmath W\rangle \quad V, W \in G .
$$

We assume that $M$ is strictly pseudoconvex, which means that $\ell_{\theta}$ is positive definite for a suitable choice of $\theta$. $\ell_{\theta}$ extends by complex linearity to $\mathbb{C} G$, and induces a hermitian form on $T_{1,0}$, which we write:

$$
\ell_{\theta}(V, \bar{W})=\langle-2 i d \theta, V \wedge \bar{W}\rangle \quad V, W \in T_{1,0} .
$$


A pseudo-hermitian structure on $M$ is a $\mathrm{CR}$ structure together with a given contact form $\theta$. With this choice, $M$ is equipped with a natural volume form $\theta \wedge d \theta^{n}$. The inner product determines an isomorphism $G \simeq G^{*}$ which in turn determines a dual form $\ell_{\theta}^{*}$ on $G^{*}$, which extends naturally to $T^{*} M$. This defines a norm $|\omega|_{\theta}$ on real 1-forms $\omega$, which satisfies:

$$
|\omega|_{\theta}^{2}=\ell_{\theta}^{*}(\omega, \omega)=2 \sum_{j=1}^{j=n}\left|\omega\left(Z_{j}\right)\right|^{2}
$$

whenever $\left\{Z_{1}, \ldots, Z_{n}\right\}$ form an orthonormal basis for $T_{1,0}$ with respect to the Levy form.

The sublaplacian operator $\Delta_{b}$ is defined on real functions $u \in \mathcal{C}^{\infty}(M)$ by:

$$
\int_{M}\left(\Delta_{b} u\right) v \theta \wedge d \theta^{n}=\int_{M} \ell_{\theta}^{*}(d u, d v) \theta \wedge d \theta^{n}, \forall v \in \mathcal{C}_{0}^{\infty}(M) .
$$

Since $|\theta|_{\theta}=0$ (even $\theta \in T_{1,0}^{\perp}$ ), $\ell_{\theta}^{*}$ is degenerate on $T^{*} M$, and so the operator $\Delta_{b}$ is subelliptic rather than elliptic. It is shown in [14] that $\Delta_{b}=\mathcal{R} e \square_{b}$, where $\square_{b}$ is the Kohn-Spencer Laplacian acting on functions [13].

Let $(M, \theta)$ be a CR compact manifold with a contact form $\theta$. We may assume that the scalar curvature of $\theta, R_{\theta}$ is strictly positive (one can assume that, with a conformal change of contact form, $R_{\theta}>0$ or $R_{\theta}=0$ or $R_{\theta}<0$ ). The Yamabe problem is easily solved for the cases $R_{\theta}=0$ and $R_{\theta}<0$.

If we replace $\theta$ by $\widetilde{\theta}=u^{\frac{2}{n}} \theta$, then the operator $L=q \Delta_{b}+R_{\theta}$ on $M$, where $q=2+\frac{2}{n}$, satisfies the transformation law:

$$
\widetilde{L}(\widetilde{v})=\left(q \widetilde{\Delta}_{b}+\widetilde{R}_{\widetilde{\theta}}\right) \widetilde{v}=u^{-\left(1+\frac{2}{n}\right)} L(v)
$$

where $\widetilde{v}=u^{-1} v$.

If we substitute $u=v$ in (1.2), we obtain the transformation law for the scalar curvature

$$
\widetilde{R}_{\widetilde{\theta}}=u^{-\left(1+\frac{2}{n}\right)}\left(q \Delta_{b}+R_{\theta}\right) u .
$$

Thus, if $\theta$ is a given contact form and $u$ a positive function on $M$, a necessary and sufficient condition for the contact form $\widetilde{\theta}=u^{\frac{2}{n}} \theta$ to have a constant scalar curvature $\widetilde{R}_{\widetilde{\theta}} \equiv \lambda$ is that $u$ satisfies:

$$
q \Delta_{b} u+R_{\theta} u=\lambda u^{1+\frac{2}{n}} .
$$

This is the CR Yamabe equation. We will assume in the sequel that $\widetilde{R}_{\widetilde{\theta}} \equiv 1$.

G.B. Folland and E.M. Stein have introduced functional spaces for CR manifolds analogous to Sobolev spaces for Riemannian manifolds, $S_{k}^{p}(M)$ called Folland and Stein spaces [8], (see also Section 4). 
Let

$$
\begin{gathered}
H=\left\{u \in S_{1}^{2}(M) \text { such that } \int_{M}|d u|_{\theta}^{2} \theta \wedge d \theta^{n}<\infty\right. \\
\text { and } \left.\int_{M}|u|^{2+\frac{2}{n}} \theta \wedge d \theta^{n}<\infty\right\} \\
\sum=\left\{u \in H, \text { such that }\|u\|_{H}=1\right\}
\end{gathered}
$$

where

$$
\|u\|_{H}=\left(\int_{M}\left(q|d u|_{\theta}^{2}+R_{\theta} u^{2}\right) \theta \wedge d \theta^{n}\right)^{\frac{1}{2}} \quad\left(q=2+\frac{2}{n}\right)
$$

and

$$
\sum_{+}=\left\{u \in \sum \mid u \geq 0\right\}
$$

For $u \in H$, we define the following functional:

$$
J(u)=\frac{\left(\int_{M}\left(q|d u|_{\theta}^{2}+R_{\theta} u^{2}\right) \theta \wedge d \theta^{n}\right)^{1+\frac{1}{n}}}{\int_{M}|u|^{2+\frac{2}{n}} \theta \wedge d \theta^{n}} .
$$

If $u$ is a critical point of $J$ on $\sum_{+}$, then $J(u)^{\frac{n}{2}} u$ is a solution of the CR Yamabe Equation (1.4).

D. Jerison and J.M. Lee in [10] used another functional:

$$
\left\{\begin{array}{l}
A_{\theta}(u)=\int_{M}\left(q|d u|_{\theta}^{2}+R_{\theta} u^{2}\right) \theta \wedge d \theta^{n} \\
\text { and } \\
B_{\theta}(u)=\int_{M}|u|^{2+\frac{2}{n}} \theta \wedge d \theta^{n} .
\end{array}\right.
$$

Note that if $\left(u_{k}\right) \in \sum_{+}$, then $J^{\prime}\left(u_{k}\right) \rightarrow 0$ if and only if $A_{\theta}^{\prime}\left(u_{k}\right) \rightarrow 0$.

\section{The Heisenberg group and normal coordinates.}

The Heisenberg group $\mathbb{H}^{n}$ is the Lie group whose underlying manifold is $\mathbb{C}^{n} \times \mathbb{R}$, with coordinates $(z, t)=\left(z^{1}, \ldots, z^{n}, t\right)$ and whose group law is given by:

$$
(z, t)\left(z^{\prime}, t^{\prime}\right)=\left(z+z^{\prime}, t+t^{\prime}+2 \mathcal{I} m . z \bar{z}^{\prime}\right)
$$

where

$$
z \overline{z^{\prime}}=\sum_{j=1}^{j=n} z^{j} \bar{z}^{j}
$$

We define a norm in $\mathbb{H}^{n}$ by

$$
\|y\|_{\mathbb{H}^{n}}=\|(z, t)\|_{\mathbb{H}^{n}}=\left(|z|^{4}+t^{2}\right)^{\frac{1}{4}}
$$


and we define dilations by

$$
y=(z, t) \mapsto \delta y=\left(\delta z, \delta^{2} t\right), \delta>0 .
$$

The vector fields

$$
Z_{j}=\frac{\partial}{\partial z^{j}}+i \bar{z}^{j} \frac{\partial}{\partial t}, j=1, \ldots, n
$$

are invariant with respect to the group multiplication on the left, and homogeneous of degree -1 with respect to the dilations. Then $T_{1,0}=$ span $\left\{Z_{1}, \ldots, Z_{n}\right\}$ gives a left invariant CR structure on $\mathbb{H}^{n}$.

The real 1-form

$$
\theta_{0}=d t+\sum_{j=1}^{j=n}\left(i z^{j} d \bar{z}^{j}-i \bar{z}^{j} d z^{j}\right)
$$

annihilates $T_{1,0}$, we take it to be the contact form for the CR structure.

Let

$$
\mathcal{L}_{0}=-\frac{1}{2} \sum_{j=1}^{j=n}\left(Z_{j} \bar{Z}_{j}+\bar{Z}_{j} Z_{j}\right) .
$$

Then the operator $\Delta_{b}$ associated to $\theta_{0}$ is $\mathcal{L}_{0}$, and the CR invariant $\lambda\left(\mathbb{H}^{n}\right)$ $=\lambda\left(S^{2 n+1}\right)($ see $[\mathbf{1 0}])$.

Whenever the scalar curvature of $\left(\mathbb{H}^{n}, \theta_{0}\right)$ is identically zero, the CR Yamabe equation in $\mathbb{H}^{n}$ is:

$$
\mathcal{L}_{0} u=\frac{n^{2}}{4} u^{1+\frac{2}{n}}
$$

D. Jerison and J.M. Lee showed in [12] that all the solutions of (2.2) are obtained from $u(z, t)=\frac{C}{\left.|t+i| z\right|^{2}+\left.\mu\right|^{n}}$, (where $C>0, \mu \in \mathbb{C}$ with $\mathcal{I} m \mu>$ $\left.0,(z, t) \in \mathbb{H}^{n}\right)$ by left translations and dilations on $\mathbb{H}^{n}$.

G.B. Folland and E.M. Stein constructed normal coordinates which show how closely the Heisenberg group approximates a general CR manifold $(M, \theta)$ : The exponential map, $\exp _{a}$ for $a \in M$, is a diffeomorphism of a neighborhood $\mathcal{U}_{a}$ of the origin in $\mathbb{H}^{n}$, onto a neighborhood $\mathcal{V}_{a}$ of $a$ in $M$, and $\exp _{a}^{-1}$ defines a system of local coordinates on $\mathcal{V}_{a}$, called normal coordinates (see [8] Theorem 14.10).

\section{The case of a CR manifold locally CR equivalent to $S^{2 n+1}$.}

We will prove the following result:

Theorem 1. Let $(M, \theta)$ be a compact $(2 n+1)$-dimensional CR manifold, locally CR equivalent to the sphere $S^{2 n+1}$, then Equation (1) has a solution.

In this section we will establish some technical results.

We first introduce the following notations and constructions: Since $M$ is compact and locally $\mathrm{CR}$ equivalent to $S^{2 n+1}$, any point $a$ of $M$ has a 
neighborhood of normal coordinates $\mathcal{U}_{a} \supset B(a, \rho)$, where $\rho$ is independent of $a$, such that the contact form of $M$ is conformal to the contact form $\theta_{0}$ of $\mathbb{H}^{n}$, defined by (2.1). Thus, there exists a positive function $\widetilde{u}_{a}$ on $B(a, \rho)$ such that

$$
\theta_{0}=\widetilde{u}_{a}^{\frac{2}{n}} \theta
$$

The proof is similar of that the Riemannian case. In the Riemannian case, it is completed, for example, in [2], Appendix C. Such a result can be easily derived from D. Jerison and J.M. Lee extensive study of the existence of CR normal coordinates [11].

Let $u_{a}$ be the following function:

$$
\begin{cases}u_{a}(x)=\omega_{a}(x) \widetilde{u}_{a}(x) & \text { on } B(a, \rho) \\ u_{a}(x)=0 & \text { on }{ }^{\mathcal{C}} B(a, \rho)\end{cases}
$$

where $\omega_{a}(x)=\chi(\|x\|): \mathbb{R}_{+} \longrightarrow[0,1]$ is a cut off function such that:

$$
\begin{cases}\chi(t)=1 & \text { if } 0 \leq t \leq \frac{\rho}{2} \\ \chi(t)=0 & \text { if } t \geq \rho\end{cases}
$$

and $\|x\|=\left\|\exp _{a}^{-1}(x)\right\|_{\mathbb{H}^{n}}$, where $\|\cdot\|_{\mathbb{H}^{n}}$ is the norm of the Heisenberg group $\mathbb{H}^{n}$ defined in Section 2.

Let $\lambda$ be a positive, large parameter.

We introduce on $B(a, \rho)$ the following function:

$$
\delta(a, \lambda)=\sigma \frac{\lambda^{n}}{\left|1+\lambda^{2}\left(|z|^{2}-i t\right)\right|^{n}}
$$

where

$$
(z, t)=\exp _{a}^{-1}(x)
$$

and, the constant $\sigma$ is such that the following equation is satisfied

$$
L_{\theta_{0}} \delta(a, \lambda)=\delta(a, \lambda)^{1+\frac{2}{n}} \quad \text { on } B(a, \rho) .
$$

We define a family of "almost solutions" $\hat{\delta}(a, \lambda)$ to be the unique solutions on $M$ of:

$$
L \hat{\delta}(a, \lambda)=\delta^{\prime}(a, \lambda)^{1+\frac{2}{n}}
$$

with

$$
\left\{\begin{array}{ll}
\delta^{\prime}(a, \lambda)(x)=u_{a}(x) \delta(a, \lambda)(x) & \text { on } B(a, \rho) \\
\delta^{\prime}(a, \lambda)(x)=0 & \text { on }{ }^{\mathcal{C}} B(a, \rho)
\end{array} .\right.
$$

We then have: 
Lemma $2([\mathbf{1 0}])$. Let $\psi$ be a function in $\mathcal{C}^{2}\left(B\left(a, \frac{\rho}{2}\right), \mathbb{R}\right)$, we have the following relation between the conformal laplacians of $M$ and $\mathbb{H}^{n}$ :

$$
L\left(u_{a} \psi\right)=u_{a}^{1+\frac{2}{n}}\left(L_{\theta_{0}} \psi\right) \quad \text { on } B\left(a, \frac{\rho}{2}\right) .
$$

For a proof one can see [10].

We prove in this section, the two following technical lemmas which are needed later, for the estimates of Section 5.

Lemma 3. There are two positive constants $C$ and $B$, such that for all a in $M$ and $\lambda \geq B$, we have:

$$
\left|\hat{\delta}(a, \lambda)-\delta^{\prime}(a, \lambda)\right| \leq \frac{C}{\lambda^{n}} \quad \text { on } B(a, \rho) .
$$

Lemma 4. Let $\widetilde{\gamma}>0$ be given. There are positive constants $C, C^{\prime}, C_{1}$ and $B$ such that if $\lambda \geq B$, we have for all $a, b$ in $M$ :

$\left.\left|\int_{M} L \hat{\delta}(a, \lambda) \cdot \hat{\delta}(a, \lambda) \theta \wedge d \theta^{n}-\sigma^{2} \int_{\mathbb{H}^{n}}\right| d\left(\frac{1}{\left|1+\left(|z|^{2}-i t\right)\right|^{n}}\right)\right|_{\theta_{0}} ^{2} \theta_{0} \wedge d \theta_{0}^{n} \mid \leq \frac{C^{\prime}}{\lambda^{2 n}}$

$$
\left|\int_{M} \hat{\delta}(a, \lambda)^{2+\frac{2}{n}} \theta \wedge d \theta^{n}-\sigma^{2+\frac{2}{n}} \int_{\mathbb{H}^{n}} \frac{1}{\left|1+\left(|z|^{2}-i t\right)\right|^{2 n+2}} \theta_{0} \wedge d \theta_{0}^{n}\right| \leq \frac{C^{\prime}}{\lambda^{2 n}}
$$

$$
\left|\int_{M} L \hat{\delta}(a, \lambda) \cdot \hat{\delta}(b, \lambda) \theta \wedge d \theta^{n}\right| \leq(1+\widetilde{\gamma}) \int_{M} \hat{\delta}(a, \lambda)^{1+\frac{2}{n}} \hat{\delta}(b, \lambda) \theta \wedge d \theta^{n}
$$

$$
\int_{M} \hat{\delta}(a, \lambda)^{1+\frac{2}{n}} \hat{\delta}(b, \lambda) \theta \wedge d \theta^{n} \geq \frac{C_{1}}{\lambda^{2 n}} .
$$

Proof of Lemma 3. We have $L \hat{\delta}(a, \lambda)=L \delta^{\prime}(a, \lambda)$ on $B\left(a, \frac{\rho}{2}\right)$ and, using Lemma 2 we obtain:

$$
\left|L \delta^{\prime}(a, \lambda)\right| \leq \frac{C}{\lambda^{n}} \quad \text { on }{ }^{\mathcal{C}} B\left(a, \frac{\rho}{2}\right) .
$$

Let $\Psi(x)=L\left(\hat{\delta}(a, \lambda)-\delta^{\prime}(a, \lambda)\right)$. Then $|\Psi(x)| \leq \frac{C^{\prime}}{\lambda^{n}}$, ( $\Psi$ is zero on $\left.{ }^{\mathcal{C}} B(a, \rho)\right)$. By the positivity of the $\mathrm{CR}$ invariant laplacian $L,\left(R_{\theta}>0\right)$, we derive the existence of a Green function $F_{L}$ associated to $L$ on $M$. Thus, we have for all $x$ in $M$ :

$$
\left(\hat{\delta}(a, \lambda)-\delta^{\prime}(a, \lambda)\right)(x)=\int_{M} F_{L}(x, y) \Psi(y) d y=\int_{B(a, \rho)} F_{L}(x, y) \Psi(y) d y
$$


and, since $F_{L}(x, y)$ is integrable in $B(a, \rho)$ (for a proof we refer to [16], [17] and $[\mathbf{1 5}])$, we obtain on $B(a, \rho)$ :

$$
\left|\hat{\delta}(a, \lambda)-\delta^{\prime}(a, \lambda)\right| \leq\|\Psi\|_{\infty} \int_{B(a, \rho)}\left|F_{L}(x, y)\right| d y \leq \frac{C}{\lambda^{n}} .
$$

Lemma 3 follows.

Proof of Lemma 4. Proof of (i): For $\xi<\frac{\rho}{2}$, we have $\delta^{\prime}(a, \lambda)=\delta(a, \lambda)$ on $B(a, \xi)$, hence, using Lemma 3 , there exists a positive constant $C$ such that:

$$
\hat{\delta}(a, \lambda)(x) \geq \frac{C}{\lambda^{n}} \forall x \in B(a, \xi) .
$$

Thus we have:

$$
\left\{\begin{array}{ll}
L \hat{\delta}(a, \lambda)(x) \geq 0 & \forall x \in B(a, \xi) \\
\hat{\delta}(a, \lambda)(x) \geq \frac{C}{\lambda^{n}} & \forall x \in \partial B(a, \xi)
\end{array} .\right.
$$

(i) follows using the maximum principle.

Proof of (ii): Denote by $B\left(0, \rho^{\prime}\right)=\exp _{a}^{-1}(B(a, \rho))$, where $0=(0,0) \in$ $\mathbb{H}^{n}$. Using Lemma 3 , we obtain:

$$
\begin{aligned}
\int_{M} L \hat{\delta}(a, \lambda) \cdot \hat{\delta}(a, \lambda) \theta \wedge d \theta^{n}= & \left(\int_{B(a, \rho)}\left(\omega_{a} \widetilde{u}_{a}\right)^{2+\frac{2}{n}} \delta(a, \lambda)^{2+\frac{2}{n}} \theta \wedge d \theta^{n}\right. \\
& \left.+O\left(\frac{1}{\lambda^{n}}\right) \int_{B(a, \rho)} \delta^{\prime}(a, \lambda)^{1+\frac{2}{n}} \theta \wedge d \theta^{n}\right) .
\end{aligned}
$$

We have

$$
\int_{B(a, \rho)} \delta^{\prime}(a, \lambda)^{1+\frac{2}{n}} \theta \wedge d \theta^{n}=\frac{C_{0}}{\lambda^{n}},
$$

where $C_{0}$ is a positive constant. Thus

$$
\begin{aligned}
& \int_{M} L \hat{\delta}(a, \lambda) \cdot \hat{\delta}(a, \lambda) \theta \wedge d \theta^{n} \\
& =\sigma^{2+\frac{2}{n}} \int_{B\left(0, \rho^{\prime}\right)} \omega_{a}^{2+\frac{2}{n}}\left(\frac{\lambda^{n}}{\left|1+\lambda^{2}\left(|z|^{2}-i t\right)\right|^{n}}\right)^{2+\frac{2}{n}} \theta_{0} \wedge d \theta_{0}^{n}+O\left(\frac{1}{\lambda^{2 n}}\right) \\
& =\sigma^{2} \int_{B\left(0, \rho^{\prime}\right)}\left|d\left(\frac{\lambda^{n}}{\left|1+\lambda^{2}\left(|z|^{2}-i t\right)\right|^{n}}\right)\right|_{\theta_{0}}^{2} \theta_{0} \wedge d \theta_{0}^{n}+O\left(\frac{1}{\lambda^{2 n}}\right)
\end{aligned}
$$

and (ii) follows since

$$
\begin{aligned}
& \int_{\mathcal{C}_{B\left(0, \rho^{\prime}\right)}}\left|d\left(\frac{\lambda^{n}}{\left|1+\lambda^{2}\left(|z|^{2}-i t\right)\right|^{n}}\right)\right|_{\theta_{0}}^{2} \theta_{0} \wedge d \theta_{0}^{n} \\
& =\int_{\mathcal{C}_{B\left(0, \lambda \rho^{\prime}\right)}}\left|d\left(\frac{1}{\left|1+\left(|z|^{2}-i t\right)\right|^{n}}\right)\right|_{\theta_{0}}^{2} \theta_{0} \wedge d \theta_{0}^{n}=O\left(\frac{1}{\lambda^{2 n}}\right) .
\end{aligned}
$$


Proof of (iii):

$$
\begin{aligned}
& \int_{M} \hat{\delta}(a, \lambda)^{2+\frac{2}{n}} \theta \wedge d \theta^{n} \\
& =\int_{M} \delta^{\prime}(a, \lambda)^{2+\frac{2}{n}} \theta \wedge d \theta^{n}+O\left(\frac{1}{\lambda^{2 n}}\right) \\
& =\int_{B(a, \rho)} \omega_{a}^{2+\frac{2}{n}} \widetilde{u}_{a}^{2+\frac{2}{n}} \delta(a, \lambda)^{2+\frac{2}{n}} \theta \wedge d \theta^{n}+O\left(\frac{1}{\lambda^{2 n}}\right) \\
& =\sigma^{2+\frac{2}{n}} \int_{B\left(0, \rho^{\prime}\right)} \omega_{a}^{2+\frac{2}{n}} \frac{\lambda^{2 n+2}}{\left|1+\lambda^{2}\left(|z|^{2}-i t\right)\right|^{2 n+2}} \theta_{0} \wedge d \theta_{0}^{n}+O\left(\frac{1}{\lambda^{2 n}}\right) \\
& =\sigma^{2+\frac{2}{n}} \int_{\mathbb{H}^{n}} \frac{1}{\left|1+\left(|z|^{2}-i t\right)\right|^{2 n+2}} \theta_{0} \wedge d \theta_{0}^{n}+O\left(\frac{1}{\lambda^{2 n}}\right)
\end{aligned}
$$

since we have:

$$
\int_{\mathcal{C}_{B\left(0, \lambda \rho^{\prime}\right)}} \frac{1}{\left|1+\left(|z|^{2}-i t\right)\right|^{2 n+2}} \theta_{0} \wedge d \theta_{0}^{n}=O\left(\frac{1}{\lambda^{2 n+2}}\right) .
$$

Proof of (v): We use (i) and we obtain:

$$
\int_{M} \hat{\delta}(a, \lambda)^{1+\frac{2}{n}} \hat{\delta}(b, \lambda) \theta \wedge d \theta^{n} \geq \frac{C}{\lambda^{n}} \int_{B(a, \rho)} \hat{\delta}(a, \lambda)^{1+\frac{2}{n}} \theta \wedge d \theta^{n}
$$

we then have, using Lemma 3 and (i), that:

$$
\begin{aligned}
\int_{M} \hat{\delta}(a, \lambda)^{1+\frac{2}{n}} \hat{\delta}(b, \lambda) \theta \wedge d \theta^{n} & \geq \frac{C}{\lambda^{n}} \int_{B\left(a, \frac{\rho}{2}\right)} \hat{\delta}(a, \lambda)^{1+\frac{2}{n}} \theta \wedge d \theta^{n} \\
& \geq \frac{C}{\lambda^{n}} \int_{B\left(a, \frac{\rho}{2}\right)} \delta^{\prime}(a, \lambda)^{1+\frac{2}{n}} \theta \wedge d \theta^{n} \\
& \geq \frac{C^{\prime \prime}}{\lambda^{n}} \int_{B\left(a, \frac{\rho}{2}\right)} \delta(a, \lambda)^{1+\frac{2}{n}} \theta \wedge d \theta^{n},
\end{aligned}
$$

where $C^{\prime \prime}$ is a suitable constant.

Since

$$
\begin{aligned}
& \int_{B(a, \rho)} \delta(a, \lambda)^{1+\frac{2}{n}} \theta \wedge d \theta^{n} \\
& =\frac{C}{\lambda^{n}} \int_{B\left(0, \lambda \rho^{\prime}\right)} \frac{1}{\left|1+\left(|z|^{2}-i t\right)\right|^{n+2}} \theta_{0} \wedge d \theta_{0}^{n} \\
& =\frac{C}{\lambda^{n}}\left\{\int_{\mathbb{H}^{n}} \frac{1}{\left|1+\left(|z|^{2}-i t\right)\right|^{n+2}} \theta_{0} \wedge d \theta_{0}^{n}+O(1)\right\},
\end{aligned}
$$

we have:

$$
\int_{M} \hat{\delta}(a, \lambda)^{1+\frac{2}{n}} \hat{\delta}(b, \lambda) \theta \wedge d \theta^{n} \geq \frac{C_{1}}{\lambda^{2 n}} .
$$

(v) follows. 
Proof of (iv): We have

$$
\begin{aligned}
& \int_{M} L \hat{\delta}(a, \lambda) \cdot \hat{\delta}(b, \lambda) \theta \wedge d \theta^{n} \\
& =\int_{B(a, \rho)} \delta^{\prime}(a, \lambda)^{1+\frac{2}{n}} \hat{\delta}(b, \lambda) \theta \wedge d \theta^{n} \\
& =\int_{B\left(a, \frac{\rho}{2}\right)} \delta^{\prime}(a, \lambda)^{1+\frac{2}{n}} \hat{\delta}(b, \lambda) \theta \wedge d \theta^{n}+\int_{B(a, \rho) \backslash B\left(a, \frac{\rho}{2}\right)} \delta^{\prime}(a, \lambda)^{1+\frac{2}{n}} \hat{\delta}(b, \lambda) \theta \wedge d \theta^{n} \\
& \leq \int_{B\left(a, \frac{\rho}{2}\right)} \delta^{\prime}(a, \lambda)^{1+\frac{2}{n}} \hat{\delta}(b, \lambda) \theta \wedge d \theta^{n}+O\left(\frac{1}{\lambda^{n+2}}\right) \int_{B(a, \rho)} \hat{\delta}(b, \lambda) \theta \wedge d \theta^{n} .
\end{aligned}
$$

Using Lemma 3 and Appendix A, we obtain:

$$
\begin{aligned}
& \int_{M} L \hat{\delta}(a, \lambda) \hat{\delta}(b, \lambda) \theta \wedge d \theta^{n} \\
& \leq\left(\int_{B(a, \rho)} \hat{\delta}(a, \lambda)^{1+\frac{2}{n}} \hat{\delta}(b, \lambda) \theta \wedge d \theta^{n}\right. \\
& \left.\quad+O\left(\frac{1}{\lambda^{n}}\right) \int_{B(a, \rho)} \delta(a, \lambda)^{\frac{2}{n}} \hat{\delta}(b, \lambda)+O\left(\frac{1}{\lambda^{n+2}}\right)\left(\int_{B(a, \rho)} \hat{\delta}(b, \lambda)^{1+\frac{2}{n}}\right)^{\frac{n}{n+2}}\right) \\
& \leq\left(\int_{M} \hat{\delta}(a, \lambda)^{1+\frac{2}{n}} \hat{\delta}(b, \lambda) \theta \wedge d \theta^{n}\right. \\
& \left.\quad+O^{\prime}\left(\frac{1}{\lambda^{n}}\right) \int_{B(a, \rho)} \delta(a, \lambda)^{\frac{2}{n}} \delta(b, \lambda) \theta \wedge d \theta^{n}+O\left(\frac{1}{\lambda^{n+2}} \frac{1}{\lambda^{n \frac{n}{n+2}}}\right)\right) .
\end{aligned}
$$

We prove in Appendix A that:

$$
\frac{1}{\lambda^{n}} \int_{B(a, \rho)} \delta(a, \lambda)^{\frac{2}{n}} \delta(b, \lambda) \theta \wedge d \theta^{n}=o\left(\int_{\mathbb{H}^{n}} \delta(a, \lambda)^{1+\frac{2}{n}} \delta(b, \lambda) \theta_{0} \wedge d \theta_{0}^{n}\right) .
$$

Thus, we have after using Lemma 3 and (v):

$$
\begin{aligned}
\int_{M} L \hat{\delta}(a, \lambda) \hat{\delta}(b, \lambda) \theta \wedge d \theta^{n} \leq & \left(\int_{M} \hat{\delta}(a, \lambda)^{1+\frac{2}{n}} \hat{\delta}(b, \lambda) \theta \wedge d \theta^{n}\right. \\
& \left.+o\left(\int_{M} \hat{\delta}(a, \lambda)^{1+\frac{2}{n}} \hat{\delta}(b, \lambda) \theta \wedge d \theta^{n}\right)\right)
\end{aligned}
$$

since $n+2+\frac{n^{2}}{n+2}>2 n$.

That is:

$$
\int_{M} L \hat{\delta}(a, \lambda) \hat{\delta}(b, \lambda) \theta \wedge d \theta^{n} \leq(1+o(1)) \int_{M} \hat{\delta}(a, \lambda)^{1+\frac{2}{n}} \hat{\delta}(b, \lambda) \theta \wedge d \theta^{n}
$$

(iv) follows. 


\section{Characterization of the sequences which violate the} Palais-Smale condition. Neighborhoods of critical points at infinity.

In order to characterize the sequences which violate the Palais-Smale condition in the Riemannian case (see for example Struwe [19]), extensive use is made of the $H_{0}^{1}$-spaces and of the orthogonal projections onto such spaces.

In the CR framework, the Palais-Smale condition bears some different features due to the fact that the boundary-value problems are not completely understood, as of now and up to our current knowledge, for the CR laplacian.

Therefore, we cannot operate as in the Yamabe Riemannian case. There are some differences and each time which $H_{0}^{1}$-spaces or projections are used in the Riemannian case, we will have here to use a direct argument. The final result is the same. In the conformal case, we will have a quite simple representation of the sequences failing to satisfy the Palais-Smale condition.

In the conformally flat case, there is some simplifications. Locally, if we change the contact form $\theta$ by a conformal factor $\widetilde{u}_{a}^{\frac{2}{n}}$ which depends differentiably on $a$, we obtain $\left(\mathbb{H}^{n}, \theta_{0}\right)$. Therefore we may use locally the usual multiplication of $\mathbb{H}^{n}$ and also the standard solutions.

We recall the following:

Folland and Stein spaces ([10], [8]). For $\xi \in M$ let $U$ be a relatively compact open subset of a normal coordinates neighborhood $\Omega_{\xi} \subset M$, with contact form $\theta$ and pseudo-hermitian frame $\left\{Z_{1}, \ldots, Z_{n}\right\}$. Let $X_{j}=\mathcal{R} e Z_{j}$ and $X_{j+n}=\mathcal{I} m Z_{j}$, for $j=1, \ldots, n$. Denote $X^{\alpha}=X_{\alpha_{1}} \cdots X_{\alpha_{k}}$ where $\alpha=\left(\alpha_{1}, \ldots, \alpha_{k}\right)$ each $\alpha_{j}$ is an integer $1 \leq \alpha_{j} \leq 2 n$, and denote $\ell(\alpha)=k$. Define the norms

$$
\|f\|_{S_{p}^{r}(M)}=\sup _{\ell(\alpha) \leq p}\left\|X^{\alpha} f\right\|_{L^{r}(U)}
$$

where for a function $g$ defined on $M$ :

$$
\|g\|_{L^{r}(U)}=\left(\int_{U}|g|^{r} \theta \wedge d \theta^{n}\right)^{\frac{1}{r}} .
$$

The Folland-Stein space $S_{p}^{r}(U)$ is defined as the completion of $\mathcal{C}_{0}^{\infty}(U)$ with respect to the norm $\|\cdot\|_{S_{p}^{r}(U)}$.

For a compact strictly pseudoconvex pseudo-hermitian manifold $M$, choose a finite open covering $U_{1}, \ldots, U_{m}$ for which each $U_{j}, 1 \leq j \leq m$, has the properties of $U$ as above.

Choose a $\mathcal{C}^{\infty}$ partition of unity $\phi_{j}$ subordinate to this covering and define the Folland-Stein space such that:

$$
S_{p}^{r}(M)=\left\{f \in L^{r}(M): \phi_{j} f \in S_{p}^{r}(U), \forall j\right\} .
$$

We then have: 
Proposition 5.5 of $[\mathbf{1 0}]$. With the notations above, $S_{p}^{r}(M) \subset L^{s}(M)$ for $\frac{1}{s} \geq \frac{1}{r}-\frac{p}{2 n+2}$ and $1<r<s<\infty$.

In this section we will use the more convenient functional of D. Jerison and J.M. Lee given in (1.6). We will return later with our functional $J$. It is simple to see that the analysis of the (P.S) sequences carried out in one framework extends to the other one.

Let $H^{+}$be the subspace of $H$ (see Section 1 ) defined by:

$$
H^{+}=\{u \in H \mid u \geq 0\} .
$$

Let $\left(u_{k}\right)$ be a sequence in $H^{+}$which satisfies:

$$
\left\{\begin{array}{l}
A_{\theta}^{\prime}\left(u_{k}\right) \underset{k \rightarrow+\infty}{\longrightarrow} 0 \\
A_{\theta}\left(u_{k}\right) \leq C \text { and } B_{\theta}\left(u_{k}\right)=1, \text { where } C \text { is a positive constant. }
\end{array}\right.
$$

Then $\left(u_{k}\right)$ admits a limit in the distributional sense.

By Propositon $5.5([\mathbf{1 0}]), S_{1}^{2}(M) \hookrightarrow L^{s}(M)$ is a compact inclusion for $1<s<q=2+\frac{2}{n}$, and the sequence $\left(u_{k}\right)$ converges to $\bar{u} \in H$ in $L^{s}(M)$. We know that $u_{k} \rightarrow \bar{u}$ in $H$, and we derive, taking $s<2+\frac{2}{n}$, that $\bar{u} \geq 0$. We then have:

Proposition 5. Let $\left(u_{k}\right)$ be a sequence of $H^{+}$satisfying (4.2) and $\bar{u} \geq$ $0, \bar{u} \not \equiv 0$. Then $\bar{u}$ is $\mathcal{C}^{\infty}$ and satisfies Equation (1).

Proof. We will prove that $\bar{u}$ satisfies Equation (1) in the distributional sense and conclude by a theorem of Brezis and Kato.

The sequence $\left(u_{k}\right)$ satisfies:

$$
\left\{\begin{array}{l}
A_{\theta}^{\prime}\left(u_{k}\right)=q \Delta_{b} u_{k}+R_{\theta} u_{k}-u_{k}^{1+\frac{2}{n}} \longrightarrow 0 \text { in } H^{-1} \\
\int_{M}\left|d u_{k}\right|_{\theta}^{2} \theta \wedge d \theta^{n}<\infty, \text { and } \int_{M}\left|u_{k}\right|^{2+\frac{2}{n}} \theta \wedge d \theta^{n}=1 .
\end{array} \quad \text { (the dual of } \mathrm{H}\right. \text { ) }
$$

Let $\varphi \in \mathcal{C}_{0}^{\infty}$. Then:

$$
\int_{M} u_{k}^{1+\frac{2}{n}} \varphi \theta \wedge d \theta^{n} \quad \text { converges to } \quad \int_{M} \bar{u}^{1+\frac{2}{n}} \varphi \theta \wedge d \theta^{n} .
$$

Indeed, there exists a constant $c$ such that:

$$
\left|u_{k}^{1+\frac{2}{n}}-\bar{u}^{1+\frac{2}{n}}\right| \leq c\left|u_{k}-\bar{u}\right|\left(\left|u_{k}\right|^{\frac{2}{n}}+|\bar{u}|^{\frac{2}{n}}\right) .
$$

By Hölder, we have:

$$
\int_{M}\left|u_{k}^{1+\frac{2}{n}}-\bar{u}^{1+\frac{2}{n}}\right| \cdot|\varphi| \theta \wedge d \theta^{n} \leq c\|\varphi\|_{\infty}\left\|u_{k}-\bar{u}\right\|_{L^{\beta}}\left\|\left(\left|u_{k}\right|^{\frac{2}{n}}+|\bar{u}|^{\frac{2}{n}}\right)\right\|_{L^{\beta^{\prime}}}
$$

where $\beta$ and $\beta^{\prime}$ are such that: $\beta<2+\frac{2}{n}$ and $\frac{1}{\beta}+\frac{1}{\beta^{\prime}}=1$.

We need

$$
\frac{2}{n} \beta^{\prime}<2+\frac{2}{n}
$$


hence

Choosing such a $\beta$, we derive that:

$$
1+\frac{1}{n}<\beta<2+\frac{2}{n} .
$$

$$
\int_{M}\left|u_{k}^{1+\frac{2}{n}}-\bar{u}^{1+\frac{2}{n}}\right| \cdot|\varphi| \theta \wedge d \theta^{n} \underset{k \rightarrow \infty}{\longrightarrow} 0 .
$$

On the other hand we have:

$$
\int_{M} \Delta_{b} u_{k} \cdot \varphi=\int_{M} u_{k} \cdot \Delta_{b} \varphi \longrightarrow \int_{M} \bar{u} \cdot \Delta_{b} \varphi=\int_{M} \Delta_{b} \bar{u} \cdot \varphi
$$

hence

$$
q \Delta_{b} u_{k}+R_{\theta} u_{k}-u_{k}^{1+\frac{2}{n}} \longrightarrow q \Delta_{b} \bar{u}+R_{\theta} \bar{u}-\bar{u}^{1+\frac{2}{n}}
$$

in the space $H^{-1}$ in the distributional sense. Thus

$$
q \Delta_{b} \bar{u}+R_{\theta} \bar{u}-\bar{u}^{1+\frac{2}{n}}=0 \quad \text { in the distributional sense. }
$$

Proposition 5 follows then from the following result of D. Jerison and J.M. Lee:

Theorem 5.16 of $[10]$. Let $U$ be a relatively compact open set in a normal coordinates neighborhood. Suppose that $f, g \in \mathcal{C}^{\infty}(U), u \geq 0$ on $U, u \in L^{q}, q=2+\frac{2}{n}$, and $\Delta_{b} u+g u=f u^{s-1}$ in the distributional sense on $U$ for some $2 \leq s \leq q$. Then $u>0$ on $U$ and $u \in \mathcal{C}^{\infty}(U)$.

We now turn to define the neighborhoods of critical points at infinity. Definition 6 ([3]). For $\epsilon>0$ and an integer $p \geq 1$, let:

$$
V(p, \epsilon)=\left\{\begin{array}{c}
u \in \sum_{+} \text {such that there exists } p \text { concentration points } \\
a_{1}, \ldots, a_{p} \text { in } M \text { and } p \text { concentrations } \lambda_{1}, \ldots, \lambda_{p}>0 \\
\text { such that }\left\|u-\frac{1}{p^{\frac{1}{2}} S^{\frac{n}{2}}} \sum_{i=1}^{i=p} \hat{\delta}\left(a_{i}, \lambda_{i}\right)\right\|_{H}<\epsilon \text {, with } \lambda_{i} \geq \frac{1}{\epsilon} \\
\text { and } \forall i \neq j, \epsilon_{i j}=\frac{\lambda_{i}}{\lambda_{j}}+\frac{\lambda_{j}}{\lambda_{i}}+\lambda_{i} \lambda_{j} \widetilde{d}\left(a_{i}, a_{j}\right)^{2} \geq \frac{1}{\epsilon}
\end{array}\right\}
$$

where $\widetilde{d}(x, y)$, if $x$ and $y$ are in a small ball of radius $\rho$, is $\left\|\exp _{x}^{-1}(y)\right\|_{\mathbb{H}^{n}}$ $\left(\|\cdot\|_{\mathbb{H}^{n}}\right.$ is the norm in $\left.\mathbb{H}^{n}\right)$, with $\exp _{x}$ the CR exponential map for the point $x$, and $\widetilde{d}(x, y)$ is equal to $\frac{\rho}{2}$ otherwise.

If $u$ is a function in $V(p, \epsilon)$, we can find an optimal approximation of $u$ by the functions $\frac{1}{p^{\frac{1}{2}} S^{\frac{n}{2}}} \sum_{i=1}^{i=p} \hat{\delta}\left(a_{i}, \lambda_{i}\right)$. Indeed, we have:

Lemma $7([4])$. For every $p \geq 1$ there exists $\epsilon>0$ which depends on $p$ such that for every $u \in \sum_{+}$satisfying:

$$
\left\|u-\frac{1}{p^{\frac{1}{2}} S^{\frac{n}{2}}} \sum_{i=1}^{i=p} \hat{\delta}\left(a_{i}, \lambda_{i}\right)\right\|_{H}<\epsilon \quad \text { with } \quad \sum_{i \neq j} \epsilon_{i j}>\frac{1}{\epsilon},
$$


the minimization problem

$$
\inf _{\left(\alpha_{i}, b_{i}, \mu_{i}\right)}\left\|u-\sum_{i=1}^{i=p} \alpha_{i} \hat{\delta}\left(b_{i}, \mu_{i}\right)\right\|_{H}
$$

has a unique solution, up to permutation on the set of indices $\{1, \ldots, p\}$.

The proof of Lemma 7 is essentially similar to A. Bahri and H. Brezis in [4] (Section 6, Proposition 9).

Let us now assume that (1) has no solution. Then we have the following characterization of the sequences failing the (P.S) condition:

Proposition 8 ([4], [3]). Let $\left(u_{k}\right)$ be a sequence of $H^{+}$satisfying (4.2). Then there exists an integer $p \geq 1$ and a sequence $\left(\epsilon_{k}\right)$, with $\epsilon_{k}>0$ and $\lim _{k \rightarrow \infty} \epsilon_{k}=0$ such that, for a subsequence of $\left(u_{k}\right)$, denoted again by $\left(u_{k}\right)$, $\frac{u_{k}}{\left\|u_{k}\right\|_{H}} \in V(p, \epsilon)$.

This proposition follows from iterated blow up around the concentration points.

Proof of Proposition 8. (1) has no solution, thus $u_{k} \rightarrow \bar{u} \equiv 0$ in $H$, and $\left(u_{k}\right)$ satisfies, after renormalization:

$$
\begin{aligned}
q \Delta_{b} u_{k}+R_{\theta} u_{k}-\left|u_{k}\right|^{\frac{2}{n}} u_{k} \longrightarrow 0 \quad \text { in the distributional sense } \\
u_{k} \rightarrow 0 \text { in } H \quad \text { and } \quad u_{k} \nrightarrow 0 \text { in } H \\
\int_{M}\left|d u_{k}\right|_{\theta}^{2} \theta \wedge d \theta^{n} \leq C, \text { and } \int_{M}\left|u_{k}\right|^{2+\frac{2}{n}} \theta \wedge d \theta^{n} \leq C, \quad C>0 .
\end{aligned}
$$

We first prove:

Lemma 9. There exists $x \in M$ such that for every real $\rho>0, \exists \delta(\rho)>0$ satisfying:

$$
\forall k, \quad \int_{B(x, \rho)}\left(q\left|d u_{k}\right|_{\theta}^{2}+R_{\theta} u_{k}^{2}\right) \theta \wedge d \theta^{n} \geq \delta(\rho) .
$$

Proof. Let us assume that for all $x$ in $M$, there is $\rho(x)>0$ such that on $B(x, \rho(x)), u_{k} \underset{H}{\longrightarrow} 0$. Since $M$ is compact this is a contradiction with our hypothesis $u_{k} \nrightarrow 0$ in $H$.

We then have:

Lemma 10. The constant $\delta(\rho)$ of Lemma 9 can be taken equal to $a_{0} S^{n}$, where $a_{0}$ is any real strictly less than 1 , and $S$ is the critical Sobolev constant of the Heisenberg group $\mathbb{H}^{n}$ introduced in (5.1).

Proof. Let $\psi_{k}: \mathbb{R}_{+} \longrightarrow[0,1]$ such that

$$
\begin{cases}\psi_{k}(s)=1 & \text { for } 0 \leq s \leq \rho_{k} \\ \psi_{k}(s)=0 & \text { for } s \geq \rho_{k}+\delta_{k}\end{cases}
$$


where $\rho<\rho_{k}<2 \rho$, and $0 \leq \delta_{k} \leq \rho$.

The function $\psi_{k}$ is continuous and $\mathcal{C}^{1}$ piecewise.

Let $\phi_{k}$ be the function defined on $M$ by $\phi_{k}(x)=\psi_{k}(\|x\|)$. We then have:

$$
\left|d \phi_{k}\right|_{\theta} \leq\left|d \psi_{k}\right|_{\theta} C(\rho) \leq \frac{\bar{C}(\rho)}{\delta_{k}} .
$$

We have, after multiplication of Equation $(*)$ by $u_{k} \phi_{k}$ :

$$
\int_{M}\left(L u_{k}\right) u_{k} \phi_{k}-\int_{M} u_{k}^{q} \phi_{k}=\left\langle\alpha_{k}, u_{k} \phi_{k}\right\rangle_{H^{-1} / H}
$$

with $\alpha_{k} \underset{H^{-1}}{\longrightarrow} 0$. Thus

$$
\begin{aligned}
& \int_{M} q\left|d u_{k}\right|_{\theta}^{2} \phi_{k}+\int_{M} q u_{k} \ell_{\theta}^{*}\left(d u_{k}, d \phi_{k}\right)+\int_{M} R_{\theta} u_{k}^{2} \phi_{k} \\
& \leq \int_{M}\left|u_{k}\right|^{q} \phi_{k}+\left\|\alpha_{k}\right\|_{H^{-1}}\left\|u_{k} \phi_{k}\right\|_{H}
\end{aligned}
$$

with

$$
\left\|u_{k} \phi_{k}\right\|_{H}=\left(\int_{M}\left(q\left|d\left(u_{k} \phi_{k}\right)\right|_{\theta}^{2}+R_{\theta}\left(u_{k} \phi_{k}\right)^{2}\right) \theta \wedge d \theta^{n}\right)^{\frac{1}{2}} .
$$

Let us remark that:

$$
\left(\int_{M}\left|d\left(u_{k} \phi_{k}\right)\right|_{\theta}^{2}\right)^{\frac{1}{2}} \leq\left(\int_{M}\left|d u_{k}\right|_{\theta}^{2} \phi_{k}^{2}\right)^{\frac{1}{2}}+\left(\int_{M} u_{k}^{2}\left|d \phi_{k}\right|_{\theta}^{2}\right)^{\frac{1}{2}} .
$$

Thus

$$
\begin{gathered}
\int_{M} q\left|d u_{k}\right|_{\theta}^{2} \phi_{k}+\int_{M} q u_{k} \ell_{\theta}^{*}\left(d u_{k}, d \phi_{k}\right)+\int_{M} R_{\theta} u_{k}^{2} \phi_{k} \\
\leq \int_{M}\left|u_{k}\right|^{q} \phi_{k}+\left\|\alpha_{k}\right\|_{H^{-1}}\left\{\left(\int_{M} R_{\theta} u_{k}^{2} \phi_{k}^{2}\right)^{\frac{1}{2}}\right. \\
\left.\quad+\left(\int_{M} q\left|d u_{k}\right|_{\theta}^{2} \phi_{k}^{2}\right)^{\frac{1}{2}}+\left(\int_{M} q u_{k}^{2}\left|d \phi_{k}\right|_{\theta}^{2}\right)^{\frac{1}{2}}\right\} .
\end{gathered}
$$

We are assuming that:

$$
\begin{gathered}
\int_{M} q\left|d u_{k}\right|_{\theta}^{2} \phi_{k}+\int_{M} R_{\theta} u_{k}^{2} \phi_{k} \geq \frac{\delta(\rho)}{2}, \\
\left\|\alpha_{k}\right\|_{H^{-1}}\left(\int_{M} q\left|d u_{k}\right|_{\theta}^{2} \phi_{k}^{2}\right)^{\frac{1}{2}} \longrightarrow 0
\end{gathered}
$$

and

$$
\left\|\alpha_{k}\right\|_{H^{-1}}\left(\int_{M} R_{\theta} u_{k}^{2} \phi_{k}^{2}\right)^{\frac{1}{2}} \longrightarrow 0
$$


this allows us to incorporate these two last terms in the first side of the inequality (4.6) and we derive:

$$
\begin{aligned}
& \left(\int_{M}\left(q\left|d u_{k}\right|_{\theta}^{2} \phi_{k}+R_{\theta} u_{k}^{2} \phi_{k}\right)\right)(1+o(1))+\int_{M} q u_{k} \ell_{\theta}^{*}\left(d u_{k}, d \phi_{k}\right) \\
& \leq \int_{M}\left|u_{k}\right|^{q} \phi_{k}+\left\|\alpha_{k}\right\|_{H^{-1}}\left(\int_{M} q u_{k}^{2}\left|d \phi_{k}\right|_{\theta}^{2}\right)^{\frac{1}{2}} .
\end{aligned}
$$

We apply a Hölder inequality to

$$
\int_{M} u_{k} \ell_{\theta}^{*}\left(d u_{k}, d \phi_{k}\right)
$$

and we derive

$$
\begin{aligned}
\int_{M} u_{k} \ell_{\theta}^{*}\left(d u_{k}, d \phi_{k}\right) & \leq\left(\int_{M}\left|d u_{k}\right|_{\theta}^{2}\right)^{\frac{1}{2}}\left(\int_{M} u_{k}^{2}\left|d \phi_{k}\right|_{\theta}^{2}\right)^{\frac{1}{2}} \\
& \leq C\left(\int_{M} u_{k}^{2}\left|d \phi_{k}\right|_{\theta}^{2}\right)^{\frac{1}{2}} \leq C \frac{\bar{C}}{\delta_{k}}\left(\int_{M} u_{k}^{2}\right)^{\frac{1}{2}} .
\end{aligned}
$$

Let us denote by $\omega_{k}$ the term $\frac{\bar{C}}{\delta_{k}}\left(\int_{M} u_{k}^{2}\right)^{\frac{1}{2}}$, and impose on $\left(\omega_{k}\right)$ to converge to zero, that is we choose $\delta_{k}$ such that $\left(\int_{M} u_{k}^{2}\right)^{\frac{1}{2}}=o\left(\delta_{k}\right)$. This is possible since $\int_{M} u_{k}^{2}$ tends to zero. We then obtain

$$
\begin{aligned}
\frac{\delta(\rho)}{2} & \leq\left(\int_{M}\left(q\left|d u_{k}\right|_{\theta}^{2} \phi_{k}+R_{\theta} u_{k}^{2} \phi_{k}\right)\right)(1+o(1)) \\
& \leq \int_{M}\left|u_{k}\right|^{q} \phi_{k}+q\left(\left\|\alpha_{k}\right\|_{H^{-1}}+C\right) \omega_{k} .
\end{aligned}
$$

Since $\omega_{k}$ tends to zero as well as $\left\|\alpha_{k}\right\|_{H^{-1}}$, (4.8) can be rewritten as:

$$
(1+o(1)) \int_{M}\left(q\left|d u_{k}\right|_{\theta}^{2} \phi_{k}+R_{\theta} u_{k}^{2} \phi_{k}\right) \leq \int_{M}\left|u_{k}\right|^{q} \phi_{k}+o(1) .
$$

Since

$$
\int_{M}\left(q\left|d u_{k}\right|_{\theta}^{2} \phi_{k}+R_{\theta} u_{k}^{2} \phi_{k}\right) \geq \delta(\rho)
$$

(4.9) yields:

$$
(1+o(1)) \int_{M}\left(q\left|d u_{k}\right|_{\theta}^{2}+R_{\theta} u_{k}^{2}\right) \phi_{k} \leq \int_{M}\left|u_{k}\right|^{q} \phi_{k} .
$$

We introduce $\gamma_{k}>0$ very small which will be chosen there after. (4.10) yields

$$
(1+o(1)) \int_{M}\left(q\left|d u_{k}\right|_{\theta}^{2}+R_{\theta} u_{k}^{2}\right) \phi_{k} \leq \int_{M}\left|u_{k}\right|^{q}\left(\phi_{k}+\gamma_{k}\right) .
$$


On the other hand we have:

$$
\lambda(M)=\lambda\left(S^{2 n+1}\right)=\inf _{u \in S_{1}^{2}(M)} \int_{M}\left(q\left|d\left(\frac{u}{\|u\|_{q}}\right)\right|_{\theta}^{2}+R_{\theta}\left(\frac{u}{\|u\|_{q}}\right)^{2}\right)
$$

which implies:

$$
\lambda\left(S^{2 n+1}\right)\|u\|_{q}^{2} \leq \int_{M}\left(q|d u|_{\theta}^{2}+R_{\theta} u^{2}\right) \theta \wedge d \theta^{n} .
$$

Applying (4.11) to the function $u=u_{k}\left(\phi_{k}+\gamma_{k}\right)^{\frac{1}{q}}$, we obtain:

$$
\begin{aligned}
& \int_{M}\left|u_{k}\right|^{q}\left(\phi_{k}+\gamma_{k}\right) \\
& \leq \lambda\left(S^{2 n+1}\right)^{-\frac{q}{2}}\left(\int_{M}\left(q\left|d\left(u_{k}\left(\phi_{k}+\gamma_{k}\right)^{\frac{1}{q}}\right)\right|_{\theta}^{2}+R_{\theta} u_{k}^{2}\left(\phi_{k}+\gamma_{k}\right)^{\frac{2}{q}}\right)\right)^{\frac{q}{2}} .
\end{aligned}
$$

Hence

$$
\begin{aligned}
& \left(\int_{M}\left(q\left|d u_{k}\right|_{\theta}^{2} \phi_{k}+R_{\theta} u_{k}^{2} \phi_{k}\right)\right)(1+o(1)) \\
& \leq \lambda\left(S^{2 n+1}\right)^{-\frac{q}{2}}\left\{\int_{M} q\left|d u_{k}\right|_{\theta}^{2}\left(\phi_{k}+\gamma_{k}\right)^{\frac{2}{q}}+\int_{M} q u_{k}^{2}\left|d\left(\left(\phi_{k}+\gamma_{k}\right)^{\frac{1}{q}}\right)\right|_{\theta}^{2}\right. \\
& \left.\quad+2 \int_{M} q u_{k}\left(\phi_{k}+\gamma_{k}\right)^{\frac{1}{q}} \ell_{\theta}^{*}\left(d u_{k}, d\left(\left(\phi_{k}+\gamma_{k}\right)^{\frac{1}{q}}\right)\right)+\int_{M} R_{\theta} u_{k}^{2}\left(\phi_{k}+\gamma_{k}\right)^{\frac{2}{q}}\right\}^{\frac{q}{2}} .
\end{aligned}
$$

We choose $\gamma_{k}$ such that:

$$
\int_{M} u_{k}^{2}\left(\phi_{k}+\gamma_{k}\right)^{\frac{2}{q}-2}\left|d \phi_{k}\right|_{\theta}^{2}=o(1)
$$

This will follow if:

$$
\int_{M} u_{k}^{2} \gamma_{k}^{\frac{2}{q}-2}\left|d \phi_{k}\right|_{\theta}^{2}=o(1), \quad \text { since } \frac{2}{q}-2<0 .
$$

Since, $\int_{M} u_{k}^{2}\left|d \phi_{k}\right|_{\theta}^{2} \leq \omega_{k}^{2}$ which tends to zero, we can find $\gamma_{k}$ tending to zero such that

$$
\int_{M} u_{k}^{2}\left(\phi_{k}+\gamma_{k}\right)^{\frac{2}{q}-2}\left|d \phi_{k}\right|_{\theta}^{2} \text { tends also to zero. }
$$

The terms

$$
\int_{M} q u_{k}^{2}\left|d\left(\left(\phi_{k}+\gamma_{k}\right)^{\frac{1}{q}}\right)\right|_{\theta}^{2}
$$

and

$$
2\left\{\int_{M} q\left|d u_{k}\right|_{\theta}^{2}\left(\phi_{k}+\gamma_{k}\right)^{\frac{2}{q}} \int_{M} q u_{k}^{2}\left|d\left(\left(\phi_{k}+\gamma_{k}\right)^{\frac{1}{q}}\right)\right|_{\theta}^{2}\right\}^{\frac{1}{2}}
$$

can be incorporated in the $o(1)$ which lies in the left hand side of (4.12). 
We derive, after some obvious simplifications:

$$
\begin{aligned}
& (1+o(1)) \int_{M}\left(q\left|d u_{k}\right|_{\theta}^{2} \phi_{k}+R_{\theta} u_{k}^{2} \phi_{k}\right) \\
& \leq \lambda\left(S^{2 n+1}\right)^{-\frac{q}{2}}\left\{\int_{M} q\left|d u_{k}\right|_{\theta}^{2} \phi_{k}^{\frac{2}{q}}+\int_{M} R_{\theta} u_{k}^{2} \phi_{k}^{\frac{2}{q}}\right\}^{\frac{q}{2}} .
\end{aligned}
$$

The condition on $\gamma_{k}$ in order to satisfy (4.13) can be rewritten as: $\omega_{k} \gamma_{k}^{\frac{1}{q}-1}=$ $o(1)$.

$\gamma_{k}=\omega_{k}$ satisfies our purpose if $\omega_{k}$ tends to zero. Therefore it suffises to choose $\delta_{k}=\left(\int_{M} u_{k}^{2}\right)^{\frac{1}{4}}$. We derive from (4.14) $\left(\phi_{k}\right.$ is zero outside of $B\left(x, \rho_{k}+\delta_{k}\right)$ and is 1 on $\left.B\left(x, \rho_{k}\right)\right)$ :

$$
\begin{aligned}
& (1+o(1)) \int_{B\left(x, \rho_{k}\right)}\left(q\left|d u_{k}\right|_{\theta}^{2}+R_{\theta} u_{k}^{2}\right) \theta \wedge d \theta^{n} \\
& \leq \lambda\left(S^{2 n+1}\right)^{-\frac{q}{2}}\left(\left\{\int_{B\left(x, \rho_{k}\right)}\left(q\left|d u_{k}\right|_{\theta}^{2}+R_{\theta} u_{k}^{2}\right) \phi_{k}^{\frac{2}{q}}\right\}^{\frac{q}{2}}\right. \\
& \left.\quad+\left\{\int_{B\left(x, \rho_{k}+\delta_{k}\right)-B\left(x, \rho_{k}\right)}\left(q\left|d u_{k}\right|_{\theta}^{2}+R_{\theta} u_{k}^{2}\right) \phi_{k}^{\frac{2}{q}}\right\}^{\frac{q}{2}}\right)
\end{aligned}
$$

thus

$$
\begin{aligned}
& (1+o(1)) \int_{B\left(x, \rho_{k}\right)}\left(q\left|d u_{k}\right|_{\theta}^{2}+R_{\theta} u_{k}^{2}\right) \theta \wedge d \theta^{n} \\
& \leq \lambda\left(S^{2 n+1}\right)^{-\frac{q}{2}}\left(\left\{\int_{B\left(x, \rho_{k}\right)}\left(q\left|d u_{k}\right|_{\theta}^{2}+R_{\theta} u_{k}^{2}\right)\right\}^{\frac{q}{2}}\right. \\
& \left.\quad+\left\{\int_{B\left(x, \rho_{k}+\delta_{k}\right)-B\left(x, \rho_{k}\right)}\left(q\left|d u_{k}\right|_{\theta}^{2}+R_{\theta} u_{k}^{2}\right)\right\}^{\frac{q}{2}}\right) \\
& =\lambda\left(S^{2 n+1}\right)^{-\frac{q}{2}}((\mathrm{I})+(\mathrm{II})) .
\end{aligned}
$$

We know that $(\mathrm{I}) \geq \delta(\rho)^{\frac{q}{2}}$, and we have two possible cases for the integral (II).

First case.

Let us assume that $(\mathrm{II})=o((\mathrm{I}))$. Then:

$$
\begin{aligned}
& (1+o(1)) \int_{B\left(x, \rho_{k}\right)}\left(q\left|d u_{k}\right|_{\theta}^{2}+R_{\theta} u_{k}^{2}\right) \\
& \leq \lambda\left(S^{2 n+1}\right)^{-\frac{q}{2}}\left\{\int_{B\left(x, \rho_{k}\right)}\left(q\left|d u_{k}\right|_{\theta}^{2}+R_{\theta} u_{k}^{2}\right)\right\}^{\frac{q}{2}}
\end{aligned}
$$


that is

$$
\begin{aligned}
\int_{B\left(x, \rho_{k}\right)}\left(q\left|d u_{k}\right|_{\theta}^{2}+R_{\theta} u_{k}^{2}\right) & \geq(1+o(1))\left(\lambda\left(S^{2 n+1}\right)^{\frac{q}{2}}\right)^{\frac{2}{q-2}} \\
& \geq a\left(\lambda\left(S^{2 n+1}\right)\right)^{n+1}=a \cdot S^{n}
\end{aligned}
$$

where $a$ is any fixed constant less than 1 .

Second case.

Let us assume that (II) $>c . \delta(\rho)^{\frac{q}{2}}$, with a fixed positive $c$, for all choices of $\rho_{k}$ in $[\rho, 2 \rho]$ with $\delta_{k}=\left(\int_{M} u_{k}^{2}\right)^{\frac{1}{4}}$. Hence, if we take $\rho_{k}^{i}=\rho+i \delta_{k}, \quad i \in \mathbb{N}$,

$$
\int_{B\left(x, \rho_{k}^{i+1}\right)-B\left(x, \rho_{k}^{i}\right)}\left(q\left|d u_{k}\right|_{\theta}^{2}+R_{\theta} u_{k}^{2}\right) \geq c^{\frac{2}{q}} \delta(\rho), \quad \forall i, \quad \forall k .
$$

Extracting subsequences we may assume that $\left(u_{k}\right)$ satisfies (4.15) for $0 \leq$ $i \leq i_{0}$, where $i_{0}$ is any fixed integer less than $\frac{\rho}{\delta_{k}}$.

Thus

$$
\begin{aligned}
& \int_{B\left(x, \rho_{k}^{i_{0}+1}\right)-B(x, \rho)}\left(q\left|d u_{k}\right|_{\theta}^{2}+R_{\theta} u_{k}^{2}\right) \\
& \geq \sum_{i=0}^{i=i_{0}} \int_{B\left(x, \rho_{k}^{i+1}\right)-B\left(x, \rho_{k}^{i}\right)}\left(q\left|d u_{k}\right|_{\theta}^{2}+R_{\theta} u_{k}^{2}\right) \\
& \geq\left(i_{0}+1\right) c^{\frac{2}{q}} \delta(\rho) .
\end{aligned}
$$

On the other hand:

$$
\int_{M}\left|d u_{k}\right|_{\theta}^{2} \leq C \quad \text { and, } \quad \int_{M} u_{k}^{2} \underset{k \rightarrow \infty}{\longrightarrow} 0 .
$$

Thus, we must have:

$$
\left(i_{0}+1\right) c^{\frac{2}{q}} \delta(\rho) \leq 2 q C .
$$

Hence, if we take $i_{0}$ violating (4.16), this case cannot occur. Since $\delta_{k}$ tends to zero, such an $i_{0}$ can be found. The second case, therefore cannot occur for all $k$ 's and the first case always takes place for some $k$. Lemma 10 follows.

We derive, from Lemma 10, that for all given $0 \leq a_{0}<1$, for all $x \in M$ satisfying Lemma 9 , there is a positive sequence $\left(\rho_{k}(x)\right)$ such that:

$$
\forall k, \quad \int_{B\left(x, \rho_{k}(x)\right)}\left(q\left|d u_{k}\right|_{\theta}^{2}+R_{\theta} u_{k}^{2}\right) \theta \wedge d \theta^{n} \geq a_{0} S^{n} .
$$

For $k$ given in $\mathbb{N}$, let us denote

$$
\rho_{k, 0}=\inf _{x \in M} \rho_{k}(x) .
$$


$M$ is compact, hence this minimum is attained. Let then $x_{k, 0} \in M$ be such that $\rho_{k, 0}=\rho_{k}\left(x_{k, 0}\right)$. We now prove that:

Lemma 11. The sequence $\left(\rho_{k, 0}\right)$ converges to zero.

Proof. Let $x$ in $M$ be such that it has no neighborhood on which $u_{k} \longrightarrow{ }_{H} 0$. We choose $\rho_{0}>0$ and take $B(x, \widetilde{\rho})$ such that $\rho_{0}<\widetilde{\rho}<2 \rho_{0}$. By the construction of Lemma 10, we have:

$$
\forall k, \quad \int_{B(x, \widetilde{\rho})}\left(q\left|d u_{k}\right|_{\theta}^{2}+R_{\theta} u_{k}^{2}\right) \theta \wedge d \theta^{n} \geq a_{0} S^{n} .
$$

We derive that

$$
\rho_{k}(x) \leq \widetilde{\rho}<2 \rho_{0} .
$$

Since $\rho_{0}$ can be chosen as small as we wish, we derive that $\rho_{k}(x) \rightarrow 0$ and thus, that $\rho_{k, 0} \rightarrow 0$. Lemma 11 follows.

Since $M$ is a compact manifold which is locally CR equivalent to $S^{2 n+1}$, every $x$ in $M$ has a neighborhood $U(x) \supset B(x, \rho)$, where normal coordinates can be defined, and where the contact form of $M$ is conformal to the contact form $\theta_{0}$ of $\mathbb{H}^{n}$ (see Section 3) ( $\rho$ is independent of $x$ ). For all $k$ in $\mathbb{N}$, we consider the function $u_{k}$ on $B\left(x_{k, 0}, \frac{\rho}{2}\right)$. Let us denote by $\widetilde{u}_{k}$ the function

$$
\widetilde{u}_{k}=u_{k} u_{x_{k, 0}}^{-1}
$$

$\left(u_{x_{k, 0}}^{\frac{2}{n}}\right.$ is the conformal factor on $\left.B\left(x_{k, 0}, \frac{\rho}{2}\right)\right)$. We then have from Lemma 2 :

$$
L_{\theta}\left(u_{x_{k, 0}} \widetilde{u}_{k}\right)=\left(u_{x_{k, 0}}^{1+\frac{2}{n}}\right) L_{\theta_{0}}\left(\widetilde{u}_{k}\right) \quad \text { in } B\left(x_{k, 0}, \frac{\rho}{2}\right) .
$$

Since $M$ is compact and since

$$
q \Delta_{b} u_{k}+R_{\theta} u_{k}-u_{k}^{1+\frac{2}{n}} \underset{H^{-1}}{\longrightarrow} 0
$$

on $M$ in the distributional sense, we derive that

$$
\left(u_{x_{k, 0}}^{1+\frac{2}{n}}\right)\left(L_{\theta_{0}}\left(\widetilde{u}_{k}\right)-\widetilde{u}_{k}^{1+\frac{2}{n}}\right) \underset{H^{-1}}{\longrightarrow} 0
$$

in $B\left(x_{k, 0}, \frac{\rho}{2}\right)$ or, using the exponential map, on the ball $B\left(0, \rho^{\prime}\right)$ in $\mathbb{H}^{n}$, for a suitable $\rho^{\prime}$.

Let $\left(\widetilde{v}_{k}\right)$ be the sequence defined by

$$
\forall k \in \mathbb{N}= \begin{cases}\widetilde{v}_{k}(X)=\omega_{x_{k, 0}}\left(\rho_{k, 0} X\right) \rho_{k, 0}^{n} \widetilde{u}_{k}\left(\rho_{k, 0} X\right) & \text { on } B\left(0, \rho_{k, 0}^{-1} \rho^{\prime}\right) \\ \widetilde{v}_{k}(X)=0 & \text { on }{ }^{c_{B}} B\left(0, \rho_{k, 0}^{-1} \rho^{\prime}\right)\end{cases}
$$

where $\omega_{x_{k, 0}}$ is the function defined in Section 3 . The sequence $\left(\widetilde{v}_{k}\right)$ is obtained by dilation of the sequence $\left(\widetilde{u}_{k}\right)$. The dilation takes place in $\mathbb{H}^{n}$, 
according to the rules of Section 2 . It is easy to see that $\left(\widetilde{v}_{k}\right)$ satisfies the same properties than $\left(\widetilde{u}_{k}\right)$, that is

$$
\begin{cases}L_{\theta_{0}} \widetilde{v}_{k}-\widetilde{v}_{k}^{1+\frac{2}{n}} \rightarrow 0 & \text { in } H^{-1}(B) \\ \int_{\mathbb{H}^{n}}\left|\widetilde{v}_{k}\right|^{2+\frac{2}{n}}<\infty & \text { and } \int_{\mathbb{H}^{n}}\left|d \widetilde{v}_{k}\right|_{\theta_{0}}^{2}<\infty\end{cases}
$$

where $B$ is any given fixed ball of $\mathbb{H}^{n}$ and $k$ is large enough.

Lemma 12. (a) The sequence $\left(\widetilde{v}_{k}\right)$ converges weakly to $\widetilde{v}$ in $H$.

(b) $\widetilde{v}_{k} \longrightarrow \widetilde{v}$ in $H(B)$ for each ball of $\mathbb{H}^{n}$ and $\widetilde{v} \not \equiv 0$, positive.

Proof. (a) is straightforward.

In order to prove (b), we first establish:

Lemma 13. The sequence $\left(\widetilde{v}_{k}-\widetilde{v}\right)$ satisfies

$$
L_{\theta_{0}}\left(\widetilde{v}_{k}-\widetilde{v}\right)-\left(\widetilde{v}_{k}-\widetilde{v}\right)^{1+\frac{2}{n}} \rightarrow 0 \text { in } H^{-1}(B)
$$

for every $B$ of $\mathbb{H}^{n}$ and

$$
\int_{\mathbb{H}^{n}}\left(\left|d\left(\widetilde{v}_{k}-\widetilde{v}\right)\right|_{\theta_{0}}^{2}+\left|\left(\widetilde{v}_{k}-\widetilde{v}\right)\right|^{2+\frac{2}{n}}\right) \leq C .
$$

Proof. First, as in the Riemannian case, it is easy to see that $\widetilde{v}$ satisfies

$$
\left\{\begin{array}{l}
L_{\theta_{0}} \widetilde{v}=\widetilde{v}^{1+\frac{2}{n}} \\
\int_{\mathbb{H}^{n}}\left(|\widetilde{v}|^{2+\frac{2}{n}}+|d \widetilde{v}|_{\theta_{0}}^{2}\right)<+\infty
\end{array}\right.
$$

$\widetilde{v}$ is also clearly non-negative.

We derive then from (4.17) and (4.18)

$$
L_{\theta_{0}}\left(\widetilde{v}_{k}-\widetilde{v}\right)-\left(\widetilde{v}_{k}^{1+\frac{2}{n}}-\widetilde{v}^{1+\frac{2}{n}}\right) \longrightarrow 0 \text { in } H^{-1}(\text { loc })
$$

and we claim that

$$
\widetilde{v}_{k}^{1+\frac{2}{n}}-\widetilde{v}^{1+\frac{2}{n}}-\left(\widetilde{v}_{k}-\widetilde{v}\right)^{1+\frac{2}{n}} \longrightarrow 0 \text { in } H^{-1}(\text { loc })
$$

Indeed, using the following inequality:

$$
|| a+\left.b\right|^{\frac{2}{n}}-|a|^{\frac{2}{n}} \mid \leq c\left(|b|^{\frac{2}{n}}+\sup \{|a|,|b|\}^{\frac{2}{n}-1}|b|\right)
$$

we have:

$$
\begin{aligned}
\widetilde{v}_{k}^{\frac{2}{n}} \widetilde{v}_{k}= & \left(\widetilde{v}_{k}-\widetilde{v}+\widetilde{v}\right)^{\frac{2}{n}}\left(\widetilde{v}_{k}-\widetilde{v}\right)+\widetilde{v}_{k}^{\frac{2}{n}} \widetilde{v} \\
= & \left(\widetilde{v}_{k}-\widetilde{v}\right)^{\frac{2}{n}}\left(\widetilde{v}_{k}-\widetilde{v}\right) \\
& +O\left(\widetilde{v}^{\frac{2}{n}}\left|\widetilde{v}_{k}-\widetilde{v}\right|+\sup \left\{\left|\widetilde{v}_{k}-\widetilde{v}\right|^{\frac{2}{n}-1}, \widetilde{v}^{\frac{2}{n}-1}\right\}\left|\widetilde{v}_{k}-\widetilde{v}\right| \widetilde{v}\right)+\widetilde{v}_{k}^{\frac{2}{n}} \widetilde{v} \\
= & \left(\widetilde{v}_{k}-\widetilde{v}\right)^{\frac{2}{n}+1}+\widetilde{v}_{k}^{\frac{2}{n}} \widetilde{v}+O\left(\widetilde{v}^{\frac{2}{n}}\left|\widetilde{v}_{k}-\widetilde{v}\right|+\left|\widetilde{v}_{k}-\widetilde{v}\right|^{\frac{2}{n}} \widetilde{v}\right) \\
= & \left(\widetilde{v}_{k}-\widetilde{v}\right)^{\frac{2}{n}+1}+\left(\widetilde{v}_{k}-\widetilde{v}+\widetilde{v}\right)^{\frac{2}{n}} \widetilde{v}+O\left(\widetilde{v}^{\frac{2}{n}}\left|\widetilde{v}_{k}-\widetilde{v}\right|+\left|\widetilde{v}_{k}-\widetilde{v}\right|^{\frac{2}{n}} \widetilde{v}\right) .
\end{aligned}
$$


Applying (4.19) again, we derive:

$$
\begin{aligned}
\widetilde{v}_{k}^{\frac{2}{n}+1}= & \left(\widetilde{v}_{k}-\widetilde{v}\right)^{\frac{2}{n}+1}+\widetilde{v}^{\frac{2}{n}+1} \\
& +O\left(\widetilde{v}\left|\widetilde{v}_{k}-\widetilde{v}\right|^{\frac{2}{n}}+\sup \left\{\left|\widetilde{v}_{k}-\widetilde{v}\right|^{\frac{2}{n}-1}, \widetilde{v}^{\frac{2}{n}-1}\right\}\right)\left|\widetilde{v}_{k}-\widetilde{v}\right| \widetilde{v} \\
& +O\left(\widetilde{v}^{\frac{2}{n}}\left|\widetilde{v}_{k}-\widetilde{v}\right|+\left|\widetilde{v}_{k}-\widetilde{v}\right|^{\frac{2}{n}} \widetilde{v}\right)
\end{aligned}
$$

thus

$$
\widetilde{v}_{k}^{\frac{2}{n}+1}=\left(\widetilde{v}_{k}-\widetilde{v}\right)^{\frac{2}{n}+1}+\widetilde{v}^{\frac{2}{n}+1}+O\left(\widetilde{v}^{\frac{2}{n}}\left|\widetilde{v}_{k}-\widetilde{v}\right|+\left|\widetilde{v}_{k}-\widetilde{v}\right|^{\frac{2}{n}} \widetilde{v}\right)
$$

i.e.,

$$
\widetilde{v}_{k}^{\frac{2}{n}+1}-\widetilde{v}^{\frac{2}{n}+1}-\left(\widetilde{v}_{k}-\widetilde{v}\right)^{\frac{2}{n}+1}=O\left(\widetilde{v}^{\frac{2}{n}}\left|\widetilde{v}_{k}-\widetilde{v}\right|+\left|\widetilde{v}_{k}-\widetilde{v}\right|^{\frac{2}{n}} \widetilde{v}\right)
$$

which, using the fact that $\widetilde{v}$ is $L^{\infty} \cap L^{2+\frac{2}{n}}$ (derived from the regularity theory), converges to 0 in $L^{2 \frac{n+1}{n+2}}\left(\mathbb{H}^{n}\right)$.

Lemma 13 follows.

We resume the proof of (b). Arguing by contradiction, since $\widetilde{v}_{k}-\widetilde{v} \nrightarrow 0$ and since $\widetilde{v}_{k}-\widetilde{v}$ satisfies all the conditions of Lemma 10, there exists a sequence $\left(\widetilde{x}_{k}, \widetilde{\rho}_{k}\right)$ such that $\widetilde{x}_{k} \in B\left(x_{k, 0}, \rho_{k, 0}\right), \widetilde{\rho}_{k}$ tends to zero and:

$$
\int_{\exp _{x_{k, 0}}^{-1} B\left(\widetilde{x}_{k}, \widetilde{\rho}_{k}\right)} q\left|d\left(\widetilde{v}_{k}-\widetilde{v}\right)\right|_{\theta_{0}}^{2} \geq \frac{1+a_{0}}{2} S^{n} .
$$

Thus,

$$
\int_{\exp _{x_{k, 0}}^{-1} B\left(\widetilde{x}_{k}, \widetilde{\rho}_{k}\right)} q\left|d \widetilde{v}_{k}\right|_{\theta_{0}}^{2} \geq a_{0} S^{n} .
$$

Hence, there exists $\widetilde{\widetilde{x}}_{k} \in B\left(x_{k, 0}, \rho_{k, 0}\right)$ such that

$$
\int_{B\left(\widetilde{\widetilde{x}}_{k}, \widetilde{\rho}_{k} \rho_{k, 0}\right)}\left(q\left|d u_{k}\right|_{\theta}^{2}+R_{\theta} u_{k}^{2}\right) \geq a_{0} S^{n} .
$$

This implies that $\rho_{k}\left(\widetilde{\widetilde{x}}_{k}\right)<\widetilde{\rho}_{k} \rho_{k, 0}<\rho_{k, 0}\left(\widetilde{\rho}_{k}\right.$ tends to zero) and contradicts the optimality of $\rho_{k, 0}$ expressed in Equation $(\mathrm{O})$. Hence, $\widetilde{v}_{k} \rightarrow \widetilde{v}$ in $H(B)$, for each ball of $\mathbb{H}^{n}$ as stable and $\widetilde{v}$ has to be non-zero since

$$
\int_{B(0,1)} q\left|d \widetilde{v}_{k}\right|_{\theta_{0}}^{2} \geq \frac{a_{0}}{2} S^{n}
$$

$\widetilde{v}$ is non-negative and, by application of the maximum principle is therefore strictly positive. Lemma 12 follows.

From the uniqueness of the positive solutions on $\mathbb{H}^{n}([\mathbf{1 0}])$, we derive the existence of $b_{1} \in \mathbb{H}^{n}$ and $\gamma_{1}>0$ such that $\widetilde{v}=\delta\left(b_{1}, \gamma_{1}\right)$, where $\delta(a, \lambda)$ is the usual solution in $\mathbb{H}^{n}$. By optimality of $\left(x_{k, 0}, \rho_{k, 0}\right)$ we see that $b_{1}=0$ and $\gamma_{1}=1$. 
We recall now that, given $(a, \lambda) \in M \times \mathbb{R}_{+}$, we have introduced in (3.3) the unique solution $\hat{\delta}$ of:

$$
\left\{\begin{array}{l}
L \hat{\delta}(a, \lambda)=\delta^{\prime}(a, \lambda)^{1+\frac{2}{n}} \\
\text { with } \delta^{\prime}(a, \lambda)=u_{a} \delta(a, \lambda) \text { in } B(a, \rho)
\end{array} .\right.
$$

We now extract from $\left(u_{k}\right)$ the "first mass" $\widetilde{v}$, that is, we consider the new sequence of functions:

$$
\begin{gathered}
u_{k}^{1}(x)=u_{k}(x)-\hat{\delta}\left(\exp _{x_{k, 0}}^{-1}\left(\rho_{k, 0} b_{1}\right), \frac{\gamma_{1}}{\rho_{k, 0}}\right)(x) \\
=\left(u_{k}-\hat{\delta}\left(x_{k, 0}, \frac{\gamma_{1}}{\rho_{k, 0}}\right)\right)(x) .
\end{gathered}
$$

Lemma 11 implies readely that $u_{k}^{1}$ when rescaled around $x_{k, 0}$ via the exponential map with the scale $\rho_{k, 0}$, will tend to zero in $H^{-1}$ of the compact subsets of $\mathbb{H}^{n}$.

We then have:

Lemma 14. The sequence $\left(u_{k}^{1}\right)$ satisfies:

$$
\left\{\begin{array}{l}
L u_{k}^{1}-\left|u_{k}^{1}\right|^{\frac{2}{n}} u_{k}^{1} \rightarrow 0 \text { in } H^{-1} \\
\int_{M}\left(q\left|d u_{k}^{1}\right|_{\theta}^{2}+R_{\theta}\left(u_{k}^{1}\right)^{2}\right) \leq C
\end{array} .\right.
$$

Up to minor technical points, the proof of Lemma 14 is similar to that of Lemma 13. We derive that $\left(u_{k}^{1}\right)$ satisfies the same properties than $\left(u_{k}\right)$, up to the fact that $\left(u_{k}^{1}\right)$ is not positive any more.

Reiterating our procedure, either $\left(u_{k}^{1}\right)$ converges strongly to zero and Proposition 8 holds or we can find a new sequence $\left(x_{k, 1}, \rho_{k, 1}\right)$ around which we can rescale $\left(u_{k}^{1}\right)$.

We then have:

Lemma 15. With a good choice of the constants $a_{0}, a_{1}$, we have

$$
\rho_{k, 1} \geq \frac{1}{2} \rho_{k, 0}
$$

Proof. Let us choose $a_{1}$ for $u_{k}^{1}$, in lieu of $a_{0}$ for $u_{k}$ such that $0<a_{0}<a_{1}<1$. Arguing by contradiction, let us assume that

$$
\rho_{k, 1}<\frac{1}{2} \rho_{k, 0}
$$

Thus, there is a point $z \in M$ such that

$$
\int_{B\left(z, \frac{1}{2} \rho_{k, 0}\right)}\left(q\left|d u_{k}^{1}\right|^{2}+R_{\theta}\left(u_{k}^{1}\right)^{2}\right) \geq a_{1} S^{n} .
$$


Let us discard immediately $\int_{B\left(z, \frac{1}{2} \rho_{k, 0}\right)} R_{\theta}\left(u_{k}^{1}\right)^{2}$ since this quantity tends to zero. Thus, we may assume using the fact that $a_{1}>a_{0}$, that

$$
\int_{B\left(z, \frac{1}{2} \rho_{k, 0}\right)} q\left|d u_{k}^{1}\right|_{\theta}^{2} \geq \frac{a_{0}+a_{1}}{2} S^{n} .
$$

Now, $u_{k}^{1}$ is $u_{k}$ deprived of $\hat{\delta}\left(x_{k, 0}, \frac{1}{\rho_{k, 0}}\right)$ which is, up to local diffeomorphisms and rescaling, essentially $v_{k}-\widetilde{v}$ in $B\left(x_{k, 0}, N \rho_{k, 0}\right)$, with $N$ as large as we will and, since $\hat{\delta}$ decreases rapidly outside of such a ball ( $N$ large), is essentially $u_{k}$ in ${ }^{\mathcal{C}} B\left(x_{k, 0}, N \rho_{k, 0}\right)$.

Clearly, $v_{k}-\widetilde{v}$ tends to zero in $H\left(B\left(x_{k, 0}, N \rho_{k, 0}\right)\right)$ and $u_{k}$ satisfies, on every ball $B$ of radius $\frac{\rho_{k, 0}}{2}$,

$$
\int_{B} q\left|d u_{k}\right|_{\theta}^{2} \leq a_{0} S^{n}
$$

Therefore, after some minor technical details, we derive that, for every $y$ of $M$,

$$
\int_{B\left(y, \frac{1}{2} \rho_{k, 0}\right)} q\left|d u_{k}^{1}\right|_{\theta}^{2} \leq \frac{3 a_{0}+a_{1}}{4} S^{n}<\frac{a_{0}+a_{1}}{2} S^{n} .
$$

A contradiction and Lemma 15 follows.

We now establish the last step in the proof of Proposition 8.

We recall that, if $a$ and $b$ are points of $M, \widetilde{d}(a, b)$ is either larger than a fixed constant $\Theta$ or, if $\widetilde{d}(a, b)<\Theta$, then $a$ and $b$ are in the same conformal local chart of $M$ and $\widetilde{d}(a, b)$ can be thought of, via the exponential map of $a$ or $b$, as $\left\|a^{-1} b\right\|_{\mathbb{H}^{n}}$ at first order.

\section{Lemma 16.}

$$
\frac{\rho_{k, 1}}{\rho_{k, 0}}+\rho_{k, 0}^{-1} \rho_{k, 1}^{-1} \widetilde{d}\left(x_{k, 0}, x_{k, 1}\right)^{2} \longrightarrow+\infty .
$$

Furthermore, the weak limit of the rescaled functions of $\left(u_{k}^{1}\right)$ around $\left(x_{k, 1}\right.$, $\left.\rho_{k, 1}\right)$ is non-negative.

Proof. If $\widetilde{d}\left(x_{k, 0}, x_{k, 1}\right)$ does not tend to zero, then the mass $\hat{\delta}$ which we substracted to $u_{k}$ is concentrated around $x_{k, 0}$ which remains away from the new point of concentration $x_{k, 1}$. Hence, since $\rho_{k, 0}^{-1}$ tends to infinity, this first mass $\hat{\delta}$ tends to zero in $H^{-1}$ of fixed balls around $x_{k, 1}$, hence a fortiori in $H^{-1}$ of tiny balls of radius of order $\rho_{k, 1}$. Thus, in this case, $\hat{\delta}$ can be forgotten in the weak limit of $\left(u_{k}^{1}\right)$, which is derived by taking the weak limit of the rescaled functions from $\left(u_{k}\right)$ around $\left(x_{k, 1}, \rho_{k, 1}\right)$. The positivity of this weak limit follows as well as the fact that

$$
\frac{\rho_{k, 1}}{\rho_{k, 0}}+\rho_{k, 0}^{-1} \rho_{k, 1}^{-1} \widetilde{d}\left(x_{k, 0}, x_{k, 1}\right)^{2} \longrightarrow+\infty .
$$


If $\widetilde{d}\left(x_{k, 0}, x_{k, 1}\right)$ tends to zero, we may consider that we are in one chart of $M$, hence in fact on $\mathbb{H}^{n}$. We then subdivide our discussion into two cases:

(1) First case. Let us assume that

$$
\frac{\rho_{k, 1}}{\rho_{k, 0}} \rightarrow \infty
$$

Then, the following function

$$
\left(\frac{\rho_{k, 1}}{\rho_{k, 0}}\right)^{n} \widetilde{v}\left(\frac{\rho_{k, 1}}{\rho_{k, 0}} y \rho_{k, 0}^{-1} x_{k, 0}^{-1} x_{k, 1}\right), \quad y \in \mathbb{H}^{n}
$$

converges to zero, since it is equal to $\widetilde{v}$ translated and dilated with a concentration tending to infinity. Thus the weak limit of $\left(u_{k}^{1}\right)$ is $\geq 0$ which is an essential point in the argument; we know also that the weak limit is a strong limit ( $\rho_{k, 1}$ is optimal) and is not zero.

(2) Second case. Let us assume that

$$
\frac{1}{2} \leq \frac{\rho_{k, 1}}{\rho_{k, 0}} \leq c,
$$

where $c$ is a positive constant. Then

$$
\left\|\rho_{k, 0}^{-1} x_{k, 0}^{-1} x_{k, 1}\right\|_{\mathbb{H}^{n}} \rightarrow \infty
$$

Indeed, assuming that for all $k \in \mathbb{N}, \rho_{k, 0}^{-1} x_{k, 0}^{-1} x_{k, 1}$ is upperbounded, we rescale $\left(u_{k}^{1}\right)$ around $\left(x_{k, 1}, \rho_{k, 1}\right)$. This is equivalent to rescaling $\left(u_{k}^{1}\right)$ around $\left(x_{k, 0}, \rho_{k, 0}\right)$ and we obtain $\widetilde{v}_{k}^{1}=\widetilde{v}_{k}-\widetilde{v}$. Since $\widetilde{v}_{k}-\widetilde{v} \rightarrow 0$ we have a contradiction $\left(\widetilde{v}_{k}^{1} \nrightarrow 0\right)$. Thus

$$
\left\|\rho_{k, 0}^{-1} x_{k, 0}^{-1} x_{k, 1}\right\|_{\mathbb{H}^{n}} \rightarrow \infty
$$

and since $\rho_{k, 1} \sim c . \rho_{k, 0}$ we have

$$
\left\|\rho_{k, 1}^{-1} x_{k, 1}^{-1} x_{k, 0}\right\|_{\mathbb{H}^{n}} \rightarrow \infty
$$

and

Thus

$$
\rho_{k, 1}^{-1} \rho_{k, 0}^{-1}\left\|x_{k, 1}^{-1} x_{k, 0}\right\|_{\mathbb{H}^{n}}^{2} \rightarrow \infty .
$$

Lemma 16 follows.

$$
\left(\frac{\rho_{k, 1}}{\rho_{k, 0}}\right)^{n} \widetilde{v}\left(\frac{\rho_{k, 1}}{\rho_{k, 0}} y \rho_{k, 1}^{-1} x_{k, 1}^{-1} x_{k, 0}\right) \rightarrow 0 .
$$

We then can state lemmas analogous to Lemmas 12, 13 and (4.18) for the sequence $\left(v_{k}^{1}\right)$ and its limit $\widetilde{v}^{1}$. It is easy, using the optimality of $\left(x_{k, 1}, \rho_{k, 1}\right)$, to prove that the new "mass" $\hat{\delta}$ is centered at $x_{k, 1}$, with concentration $\frac{1}{\rho_{k, 1}}$.

This argument can be iterated as long as the new sequence obtained does not converge strongly to zero. The iteration has to stop for energy reasons: 
After each extraction of a "mass", $\int_{M} q\left|d u_{k}^{\ell}\right|_{\theta}^{2}$ (at the $\ell^{\text {th }}$-step) decreases of $S^{n}$.

Then, there exists $p \in \mathbb{N}, p \geq 1$ and sequences $\left(x_{k, 0}\right),\left(x_{k, 1}\right), \ldots,\left(x_{k, p-1}\right)$ in $M$ and $\left(\frac{1}{\rho_{k, 0}}\right)=\left(\lambda_{0}^{k}\right), \ldots,\left(\frac{1}{\rho_{k, p-1}}\right)=\left(\lambda_{p-1}^{k}\right)$ tending to $+\infty$ such that

$$
\left\|u_{k}-\sum_{i=0}^{p-1} \hat{\delta}\left(x_{k, i}, \rho_{k, i}^{-1}\right)\right\|_{H} \rightarrow 0
$$

and, for $i \neq j$

$$
\epsilon_{i, j}=\frac{\rho_{k, i}}{\rho_{k, j}}+\frac{\rho_{k, j}}{\rho_{k, i}}+\frac{1}{\rho_{k, i} \rho_{k, j}} \widetilde{d}\left(x_{k, i}, x_{k, j}\right)^{2} \longrightarrow+\infty .
$$

Proposition 8 follows, since we have

$$
\left\|u_{k}\right\|_{H}^{2} \longrightarrow\left\|\sum_{i=0}^{p-1} \hat{\delta}\left(x_{k, i}, \rho_{k, i}^{-1}\right)\right\|_{H}^{2}=p S^{n}+o(1)
$$

where $o(1)=O\left(\sum_{i \neq j} \frac{1}{\left(\epsilon_{i, j}\right)^{n}}\right) \longrightarrow 0$.

\section{Expansion of the functional near the sets of potential critical points at infinity.}

We consider the functional $J$ introduced in (1.5) for $u$ in $H$, and the Sobolev constant of $\mathbb{H}^{n}$ defined by:

$$
S=\frac{\left(\int_{\mathbb{H}^{n}}\left|d\left(\frac{1}{\left|1+\left(|z|^{2}-i t\right)\right|^{n}}\right)\right|_{\theta_{0}}^{2} \theta_{0} \wedge d \theta_{0}^{n}\right)^{1+\frac{1}{n}}}{\int_{\mathbb{H}^{n}} \frac{1}{\left|1+\left(|z|^{2}-i t\right)\right|^{2 n+2}} \theta_{0} \wedge d \theta_{0}^{n}} .
$$

We refer to the introduction for the aim of this section.

We have the following lemma:

Lemma 17. (i) $\forall p \in \mathbb{N}^{*}, \forall \epsilon>0, \exists \lambda_{p}=\lambda(p, \epsilon)$ such that for any $\left(\alpha_{1}, \ldots, \alpha_{p}\right)$ satisfying $\alpha_{i} \geq 0, \sum_{i=1}^{p} \alpha_{i}=1$, for any $\left(x_{1}, \ldots, x_{p}\right) \in$ $M^{p}$, for any $\lambda \geq \lambda_{p}$ the following inequality holds:

$$
J\left(\sum_{i=1}^{p} \alpha_{i} \hat{\delta}\left(x_{i}, \lambda\right)\right) \leq p^{\frac{1}{n}} S \quad \text { if } \quad \sum_{i \neq j} \int_{M} \hat{\delta}\left(x_{i}, \lambda\right)^{1+\frac{2}{n}} \hat{\delta}\left(x_{j}, \lambda\right) \theta \wedge d \theta^{n} \geq \epsilon .
$$

(ii) There exist $0<\beta<1, C>0$ and $\epsilon^{\prime}>0$ such that for all $p \in \mathbb{N}^{*}$, for any $\left(\alpha_{1}, \ldots, \alpha_{p}\right)$ satisfying: $\alpha_{i} \geq 0, \sum_{i=1}^{p} \alpha_{i}=1, \frac{\alpha_{i}}{\alpha_{j}} \geq \beta \forall i, j$, for 
any $\left(x_{1}, \ldots, x_{p}\right) \in M^{p}$, for any $\lambda \geq 1$ we have the following

$$
J\left(\sum_{i=1}^{p} \alpha_{i} \hat{\delta}\left(x_{i}, \lambda\right)\right) \leq \frac{\left(\sum_{i=1}^{p} \alpha_{i}^{2}\right)^{1+\frac{1}{n}}}{\sum_{i=1}^{p} \alpha_{i}^{2+\frac{2}{n}}} S\left(1+\frac{C}{\lambda^{2 n}}-\frac{p+1}{C \lambda^{2 n}}\right)
$$

provided

$$
\sum_{i \neq j} \int_{M} \hat{\delta}\left(x_{i}, \lambda\right)^{1+\frac{2}{n}} \hat{\delta}\left(x_{j}, \lambda\right) \theta \wedge d \theta^{n} \leq \epsilon^{\prime} .
$$

(iii) If in (ii) we drop the condition $\frac{\alpha_{i}}{\alpha_{j}} \geq \beta$, then we have the following inequality:

$$
\begin{aligned}
J\left(\sum_{i=1}^{p} \alpha_{i} \hat{\delta}\left(x_{i}, \lambda\right)\right) \leq \frac{\left(\sum_{i=1}^{p} \alpha_{i}^{2}\right)^{1+\frac{1}{n}}}{\sum_{i=1}^{p} \alpha_{i}^{2+\frac{2}{n}}} S\left\{1+O\left(\frac{1}{\lambda^{2 n}}\right)\right. \\
\left.\quad+\frac{1}{C} \sum_{i \neq j} \int_{M} \hat{\delta}\left(x_{i}, \lambda\right)^{1+\frac{2}{n}} \hat{\delta}\left(x_{j}, \lambda\right) \theta \wedge d \theta^{n}\right\} .
\end{aligned}
$$

Proof.

Proof of (i). We have

$$
\begin{aligned}
& J\left(\sum_{i=1}^{p} \alpha_{i} \hat{\delta}\left(x_{i}, \lambda\right)\right) \\
& \leq\left(\frac{\int_{M} \sum_{i} \alpha_{i} \delta^{\prime}\left(x_{i}, \lambda\right)^{2+\frac{2}{n}}}{\int_{M} \sum_{i} \alpha_{i} \hat{\delta}\left(x_{i}, \lambda\right)^{2+\frac{2}{n}}}\right)^{\frac{1}{2}}\left(\sum_{i=1}^{p} \int_{M} a_{i}^{\prime} \delta^{\prime}\left(x_{i}, \lambda\right)^{2+\frac{2}{n}}\right)^{\frac{1}{n}}
\end{aligned}
$$

where

$$
\left\{\begin{array}{ll}
a_{i}^{\prime}=\frac{\alpha_{i} \delta^{\prime}\left(x_{i}, \lambda\right)}{\sum_{j=1}^{p} \alpha_{j} \delta^{\prime}\left(x_{j}, \lambda\right)} & \text { if } \quad \delta^{\prime}\left(x_{i}, \lambda\right) \neq 0 \\
a_{i}^{\prime}=1 & \text { if } \delta^{\prime}\left(x_{i}, \lambda\right)=0
\end{array} .\right.
$$

This inequality is an equivalent of Lemma B.2 in [5].

Let

$$
A=\frac{\int_{M} \sum_{i} \alpha_{i} \delta^{\prime}\left(x_{i}, \lambda\right)^{2+\frac{2}{n}}}{\int_{M} \sum_{i} \alpha_{i} \hat{\delta}\left(x_{i}, \lambda\right)^{2+\frac{2}{n}}} .
$$

We have

$$
\begin{aligned}
A & =\frac{\int_{M} \sum_{i} \alpha_{i} \delta^{\prime}\left(x_{i}, \lambda\right)^{2+\frac{2}{n}}-\int_{M} \sum_{i} \alpha_{i} \hat{\delta}\left(x_{i}, \lambda\right)^{2+\frac{2}{n}}+\int_{M} \sum_{i} \alpha_{i} \hat{\delta}\left(x_{i}, \lambda\right)^{2+\frac{2}{n}}}{\int_{M} \sum_{i} \alpha_{i} \hat{\delta}\left(x_{i}, \lambda\right)^{2+\frac{2}{n}}} \\
& =1+\frac{\int_{M} \sum_{i} \alpha_{i} \delta^{\prime}\left(x_{i}, \lambda\right)^{2+\frac{2}{n}}-\int_{M} \sum_{i} \alpha_{i} \hat{\delta}\left(x_{i}, \lambda\right)^{2+\frac{2}{n}}}{\int_{M} \sum_{i} \alpha_{i} \hat{\delta}\left(x_{i}, \lambda\right)^{2+\frac{2}{n}}} .
\end{aligned}
$$

Since $\int_{M} \delta^{\prime}\left(x_{i}, \lambda\right)^{1+\frac{2}{n}} \theta \wedge d \theta^{n}=O\left(\frac{1}{\lambda^{n}}\right)$, we obtain that $|A| \leq 1+O\left(\frac{1}{\lambda^{n}}\right)$. 
We also have $\int_{M} \delta^{\prime}\left(x_{i}, \lambda\right)^{2+\frac{2}{n}} \theta \wedge d \theta^{n}=S^{n}+O\left(\frac{1}{\lambda^{2 n}}\right)$.

Let $B=\int_{M}\left(\sum_{i=1}^{p} a_{i}^{\prime} \delta^{\prime}\left(x_{i}, \lambda\right)^{2+\frac{2}{n}}\right) \theta \wedge d \theta^{n}$, we have

$$
\begin{aligned}
B=\int_{M}\left(\frac{\alpha_{1} \delta^{\prime}\left(x_{1}, \lambda\right) \delta^{\prime}\left(x_{1}, \lambda\right)^{2+\frac{2}{n}}}{\sum_{j=1}^{p} \alpha_{j} \delta^{\prime}\left(x_{j}, \lambda\right)}\right) \theta \wedge d \theta^{n} \\
+\int_{M}\left(\frac{\sum_{i=2}^{p} \alpha_{i} \delta^{\prime}\left(x_{i}, \lambda\right) \delta^{\prime}\left(x_{i}, \lambda\right)^{2+\frac{2}{n}}}{\sum_{j=1}^{p} \alpha_{j} \delta^{\prime}\left(x_{j}, \lambda\right)}\right) \theta \wedge d \theta^{n} .
\end{aligned}
$$

Since $\alpha_{j} \geq 0$ and $\delta^{\prime}\left(x_{j}, \lambda\right) \geq 0 \forall j$, we derive

$$
\frac{\alpha_{1} \delta^{\prime}\left(x_{1}, \lambda\right)}{\sum_{j=1}^{p} \alpha_{j} \delta^{\prime}\left(x_{j}, \lambda\right)} \leq \frac{\alpha_{1} \delta^{\prime}\left(x_{1}, \lambda\right)}{\alpha_{1} \delta^{\prime}\left(x_{1}, \lambda\right)+\alpha_{2} \delta^{\prime}\left(x_{2}, \lambda\right)}
$$

and thus

$$
B \leq(p-1) S^{n}+O_{p}\left(\frac{1}{\lambda^{2 n}}\right)+\int_{M} \frac{\alpha_{1} \delta^{\prime}\left(x_{1}, \lambda\right) \delta^{\prime}\left(x_{1}, \lambda\right)^{2+\frac{2}{n}}}{\alpha_{1} \delta^{\prime}\left(x_{1}, \lambda\right)+\alpha_{2} \delta^{\prime}\left(x_{2}, \lambda\right)} \theta \wedge d \theta^{n} .
$$

Reordering the functions $\hat{\delta}\left(x_{i}, \lambda\right), 1 \leq i \leq p$, we may assume that:

1)

$$
\begin{aligned}
& \int_{M}\left(\hat{\delta}\left(x_{1}, \lambda\right)^{1+\frac{2}{n}} \hat{\delta}\left(x_{2}, \lambda\right)+\hat{\delta}\left(x_{1}, \lambda\right) \hat{\delta}\left(x_{2}, \lambda\right)^{1+\frac{2}{n}}\right) \theta \wedge d \theta^{n} \\
& =\operatorname{Sup}_{\substack{1 \leq i, j \leq p \\
i \neq j}} \int_{M}\left(\hat{\delta}\left(x_{i}, \lambda\right)^{1+\frac{2}{n}} \hat{\delta}\left(x_{j}, \lambda\right)+\hat{\delta}\left(x_{i}, \lambda\right) \hat{\delta}\left(x_{j}, \lambda\right)^{1+\frac{2}{n}}\right) \theta \wedge d \theta^{n} .
\end{aligned}
$$

2) $\frac{\alpha_{1}}{\alpha_{2}} \leq 1$

We find under these hypotheses

$$
\begin{aligned}
J\left(\sum_{i=1}^{p} \alpha_{i} \hat{\delta}\left(x_{i}, \lambda\right)\right) \leq & \left(1+O\left(\frac{1}{\lambda^{n}}\right)\right)^{\frac{1}{2}}\left\{(p-1) S^{n}\right. \\
& \left.+O_{p}\left(\frac{1}{\lambda^{2 n}}\right)+\int_{B\left(x_{1}, \frac{\rho}{2}\right)} \frac{\delta^{\prime}\left(x_{1}, \lambda\right) \delta^{\prime}\left(x_{1}, \lambda\right)^{2+\frac{2}{n}}}{\delta^{\prime}\left(x_{1}, \lambda\right)+\delta^{\prime}\left(x_{2}, \lambda\right)}\right\}^{\frac{1}{n}} .
\end{aligned}
$$

Suppose first that the distance between $x_{1}$ and $x_{2}$ is less than $\frac{\rho}{2}$, then $\omega_{x_{1}}=1$ on $B\left(x_{1}, \frac{\rho}{2}\right)$, we derive:

$$
\left(\frac{\delta^{\prime}\left(x_{1}, \lambda\right)}{\delta^{\prime}\left(x_{1}, \lambda\right)+\delta^{\prime}\left(x_{2}, \lambda\right)}\right)(x)=\left(\frac{\widetilde{u}_{x_{1}} \delta\left(x_{1}, \lambda\right)}{\widetilde{u}_{x_{2}}}\right)(x) \quad \forall x \in B\left(x_{1}, \frac{\rho}{2}\right)
$$

and there exists a constant $\eta>\frac{\rho}{2}>0$ such that

$$
\frac{1}{2} \leq \frac{u_{x_{1}}}{u_{x_{2}}} \leq 2 \text { on } B\left(x_{1}, \eta\right) \text {. }
$$


We consider two cases.

First case. If $\widetilde{d}\left(x_{1}, x_{2}\right)<\eta$, then we have:

$$
\begin{aligned}
J\left(\sum_{i=1}^{p} \alpha_{i} \hat{\delta}\left(x_{i}, \lambda\right)\right) \leq & \left(1+O\left(\frac{1}{\lambda^{n}}\right)\right)\left\{(p-1) S^{n}+O_{p}\left(\frac{1}{\lambda^{2 n}}\right)\right. \\
& \left.+\int_{B\left(x_{1}, \eta\right)} \frac{2 \delta\left(x_{1}, \lambda\right) \delta^{\prime}\left(x_{1}, \lambda\right)^{2+\frac{2}{n}}}{2 \delta\left(x_{1}, \lambda\right)+\delta\left(x_{2}, \lambda\right)} \theta \wedge d \theta^{n}\right\}^{\frac{1}{n}} \\
\leq & \left(1+O\left(\frac{1}{\lambda^{n}}\right)\right)\left\{(p-1) S^{n}+O_{p}\left(\frac{1}{\lambda^{2 n}}\right)\right. \\
& \left.+\int_{\mathbb{H}^{n}} \frac{2 \delta\left(x_{1}, \lambda\right) \delta\left(x_{1}, \lambda\right)^{2+\frac{2}{n}}}{2 \delta\left(x_{1}, \lambda\right)+\delta\left(x_{2}, \lambda\right)} \theta_{0} \wedge d \theta_{0}^{n}\right\}^{\frac{1}{n}} .
\end{aligned}
$$

For a given $\epsilon_{0}>0$, let:

$$
A_{0}=\left\{x \in \mathbb{H}^{n} \text { such that } \delta\left(x_{1}, \lambda\right)(x) \geq \epsilon_{0}\left(2 \delta\left(x_{1}, \lambda\right)+\delta\left(x_{2}, \lambda\right)\right)(x)\right\},
$$

we have

$$
\begin{aligned}
\int_{\mathbb{H}^{n}} \frac{\delta\left(x_{2}, \lambda\right)}{2 \delta\left(x_{1}, \lambda\right)+\delta\left(x_{2}, \lambda\right)} \delta\left(x_{1}, \lambda\right)^{2+\frac{2}{n}} & \theta_{0} \wedge d \theta_{0}^{n} \\
& \geq \epsilon_{0} \int_{A_{0}} \delta\left(x_{2}, \lambda\right) \delta\left(x_{1}, \lambda\right)^{1+\frac{2}{n}} \theta_{0} \wedge d \theta_{0}^{n}
\end{aligned}
$$

and, on ${ }^{\mathcal{C}} A_{0}$, we have $\delta\left(x_{1}, \lambda\right) \leq \frac{\epsilon_{0}}{1-2 \epsilon_{0}} \delta\left(x_{2}, \lambda\right)$, then

$$
\begin{aligned}
& \int_{\mathbb{H}^{n}} \frac{\delta\left(x_{2}, \lambda\right)}{2 \delta\left(x_{1}, \lambda\right)+\delta\left(x_{2}, \lambda\right)} \delta\left(x_{1}, \lambda\right)^{2+\frac{2}{n}} \theta_{0} \wedge d \theta_{0}^{n} \\
& \geq \epsilon_{0}\left(\int_{\mathbb{H}^{n}} \delta\left(x_{1}, \lambda\right)^{1+\frac{2}{n}} \delta\left(x_{2}, \lambda\right)-\int_{\mathcal{C}_{A_{0}}} \delta\left(x_{1}, \lambda\right)^{1+\frac{2}{n}} \delta\left(x_{2}, \lambda\right)\right) \\
& \geq \epsilon_{0}\left(\int_{\mathbb{H}^{n}} \delta\left(x_{1}, \lambda\right)^{1+\frac{2}{n}} \delta\left(x_{2}, \lambda\right)-\left(\frac{\epsilon_{0}}{1-2 \epsilon_{0}}\right)^{\frac{1}{n}} \int_{\mathbb{H}^{n}} \delta\left(x_{1}, \lambda\right)^{1+\frac{1}{n}} \delta\left(x_{2}, \lambda\right)^{1+\frac{1}{n}}\right) \\
& =\epsilon_{0}\left(\frac{1}{2}\left\{\int_{\mathbb{H}^{n}}\left(\delta\left(x_{1}, \lambda\right)^{1+\frac{2}{n}} \delta\left(x_{2}, \lambda\right)+\delta\left(x_{2}, \lambda\right)^{1+\frac{2}{n}} \delta\left(x_{1}, \lambda\right)\right)\right\}\right. \\
& \left.\quad-\left(\frac{\epsilon_{0}}{1-2 \epsilon_{0}}\right)^{\frac{1}{n}} \frac{1}{2}\left\{\int_{\mathbb{H}^{n}}\left(\delta\left(x_{1}, \lambda\right)^{1+\frac{2}{n}} \delta\left(x_{2}, \lambda\right)+\delta\left(x_{2}, \lambda\right)^{1+\frac{2}{n}} \delta\left(x_{1}, \lambda\right)\right)\right\}\right)
\end{aligned}
$$


we obtain

$$
\begin{aligned}
& \int_{\mathbb{H}^{n}} \frac{\delta\left(x_{2}, \lambda\right)}{2 \delta\left(x_{1}, \lambda\right)+\delta\left(x_{2}, \lambda\right)} \delta\left(x_{1}, \lambda\right)^{2+\frac{2}{n}} \theta_{0} \wedge d \theta_{0}^{n} \\
& \geq \frac{\epsilon_{0}}{2}\left(1-\left(\frac{\epsilon_{0}}{1-2 \epsilon_{0}}\right)^{\frac{1}{n}}\right) \int_{\mathbb{H}^{n}}\left(\delta\left(x_{1}, \lambda\right)^{1+\frac{2}{n}} \delta\left(x_{2}, \lambda\right)\right. \\
& \left.\quad+\delta\left(x_{2}, \lambda\right)^{1+\frac{2}{n}} \delta\left(x_{1}, \lambda\right)\right) \theta_{0} \wedge d \theta_{0}^{n}
\end{aligned}
$$

Thus, if $\epsilon_{0}$ is small enough, we have $\left(1-\left(\frac{\epsilon_{0}}{1-2 \epsilon_{0}}\right)^{\frac{1}{n}}\right) \geq \frac{1}{2}$, and

$$
\begin{aligned}
& \int_{\mathbb{H}^{n}} \frac{\delta\left(x_{2}, \lambda\right)}{2 \delta\left(x_{1}, \lambda\right)+\delta\left(x_{2}, \lambda\right)} \delta\left(x_{1}, \lambda\right)^{2+\frac{2}{n}} \theta_{0} \wedge d \theta_{0}^{n} \\
& \geq \frac{\epsilon_{0}}{4}\left(\int_{\exp _{x_{1}}^{-1} B\left(x_{1}, \frac{\rho}{2}\right)} \delta\left(x_{1}, \lambda\right)^{1+\frac{2}{n}} \delta\left(x_{2}, \lambda\right) \theta_{0} \wedge d \theta_{0}^{n}\right. \\
& \left.\quad+\int_{\exp _{x_{2}}^{-1} B\left(x_{2}, \frac{\rho}{2}\right)} \delta\left(x_{1}, \lambda\right) \delta\left(x_{2}, \lambda\right)^{1+\frac{2}{n}} \theta_{0} \wedge d \theta_{0}^{n}\right) .
\end{aligned}
$$

Hence,

$$
\begin{aligned}
& \int_{\mathbb{H}^{n}} \frac{\delta\left(x_{2}, \lambda\right)}{2 \delta\left(x_{1}, \lambda\right)+\delta\left(x_{2}, \lambda\right)} \delta\left(x_{1}, \lambda\right)^{2+\frac{2}{n}} \theta_{0} \wedge d \theta_{0}^{n} \\
& \geq \frac{\epsilon_{0}}{4}\left(\int_{B\left(x_{1}, \frac{\rho}{2}\right)} \hat{\delta}\left(x_{1}, \lambda\right)^{1+\frac{2}{n}} \hat{\delta}\left(x_{2}, \lambda\right) \theta \wedge d \theta^{n}\right. \\
& \left.\quad+\int_{B\left(x_{2}, \frac{\rho}{2}\right)} \hat{\delta}\left(x_{1}, \lambda\right) \hat{\delta}\left(x_{2}, \lambda\right)^{1+\frac{2}{n}} \theta \wedge d \theta^{n}+O\left(\frac{1}{\lambda^{2 n}}\right)\right) \\
& \geq \frac{\epsilon_{0}}{4}\left(\int _ { M } \left(\hat{\delta}\left(x_{1}, \lambda\right)^{1+\frac{2}{n}} \hat{\delta}\left(x_{2}, \lambda\right) \theta \wedge d \theta^{n}\right.\right. \\
& \left.\left.\quad+\hat{\delta}\left(x_{1}, \lambda\right) \hat{\delta}\left(x_{2}, \lambda\right)^{1+\frac{2}{n}}\right) \theta \wedge d \theta^{n}+O\left(\frac{1}{\lambda^{2 n}}\right)\right)
\end{aligned}
$$

we have assumed that

$$
\begin{aligned}
& \int_{M}\left(\hat{\delta}\left(x_{1}, \lambda\right)^{1+\frac{2}{n}} \hat{\delta}\left(x_{2}, \lambda\right)+\hat{\delta}\left(x_{1}, \lambda\right) \hat{\delta}\left(x_{2}, \lambda\right)^{1+\frac{2}{n}}\right) \theta \wedge d \theta^{n} \\
& =\operatorname{Sup}_{\substack{1 \leq i, j \leq p \\
i \neq j}} \int_{M}\left(\hat{\delta}\left(x_{i}, \lambda\right)^{1+\frac{2}{n}} \hat{\delta}\left(x_{j}, \lambda\right)+\hat{\delta}\left(x_{i}, \lambda\right) \hat{\delta}\left(x_{j}, \lambda\right)^{1+\frac{2}{n}}\right) \theta \wedge d \theta^{n}
\end{aligned}
$$


then

$$
\begin{aligned}
& \sum_{i \neq j} \int_{M}\left(\hat{\delta}\left(x_{i}, \lambda\right)^{1+\frac{2}{n}} \hat{\delta}\left(x_{j}, \lambda\right)+\hat{\delta}\left(x_{i}, \lambda\right) \hat{\delta}\left(x_{j}, \lambda\right)^{1+\frac{2}{n}}\right) \theta \wedge d \theta^{n} \\
& \leq p^{2}\left(\int_{M}\left(\hat{\delta}\left(x_{i}, \lambda\right)^{1+\frac{2}{n}} \hat{\delta}\left(x_{j}, \lambda\right)+\hat{\delta}\left(x_{i}, \lambda\right) \hat{\delta}\left(x_{j}, \lambda\right)^{1+\frac{2}{n}}\right) \theta \wedge d \theta^{n}\right)
\end{aligned}
$$

and

$$
\begin{aligned}
J\left(\sum_{i=1}^{p} \alpha_{i} \hat{\delta}\left(x_{i}, \lambda\right)\right) \leq & \left(1+O\left(\frac{1}{\lambda^{n}}\right)\right)\left\{p S^{n}+O_{p}\left(\frac{1}{\lambda^{2 n}}\right)\right. \\
& \left.-C \frac{\epsilon_{0}}{p^{2}} \int_{M} \sum_{i \neq j} \hat{\delta}\left(x_{i}, \lambda\right)^{1+\frac{2}{n}} \hat{\delta}\left(x_{j}, \lambda\right)\right\}^{\frac{1}{n}} .
\end{aligned}
$$

That is

$$
J\left(\sum_{i=1}^{p} \alpha_{i} \hat{\delta}\left(x_{i}, \lambda\right)\right) \leq p^{\frac{1}{n}} S\left(1+O_{p}\left(\frac{1}{\lambda^{n}}\right)-C^{\prime \prime} \frac{\epsilon_{0} \epsilon}{p^{3}}\right)
$$

and (i) follows in this case.

Second case. If $\widetilde{d}\left(x_{1}, x_{2}\right) \geq \eta$, then

$$
\int_{M}\left(\hat{\delta}\left(x_{1}, \lambda\right)^{1+\frac{2}{n}} \hat{\delta}\left(x_{2}, \lambda\right)+\hat{\delta}\left(x_{1}, \lambda\right) \hat{\delta}\left(x_{2}, \lambda\right)^{1+\frac{2}{n}}\right) \theta \wedge d \theta^{n} \text { is } O\left(\frac{1}{\lambda^{2 n}}\right)
$$

then

$$
\begin{aligned}
& \sum_{i \neq j} \int_{M}\left(\hat{\delta}\left(x_{i}, \lambda\right)^{1+\frac{2}{n}} \hat{\delta}\left(x_{j}, \lambda\right)+\hat{\delta}\left(x_{i}, \lambda\right) \hat{\delta}\left(x_{j}, \lambda\right)^{1+\frac{2}{n}}\right) \theta \wedge d \theta^{n} \\
& \leq p^{2} O\left(\frac{1}{\lambda^{2 n}}\right)=O_{p}^{\prime}\left(\frac{1}{\lambda^{2 n}}\right) .
\end{aligned}
$$

Then, we need only to choose $\lambda$ large enough to have $O_{p}^{\prime}\left(\frac{1}{\lambda^{2 n}}\right)<\epsilon$ and the proof of (i) reduces to the case $\widetilde{d}\left(x_{1}, x_{2}\right)<\eta$.

For the proof of (ii) and (iii), we will use the following result:

Lemma 18 ([4, Lemma A.2, Appendix 2]). Let $s>2$ be given. There is a constant $\gamma(s)>1$ such that, for any $a_{1}, \ldots, a_{p} \geq 0$, we have:

$$
\left(\sum_{i=1}^{p} a_{i}\right)^{s} \geq \sum_{i=1}^{p} a_{i}^{s}+s \frac{\gamma}{2} \sum_{i \neq j} a_{i}^{s-1} a_{j} .
$$


Proof of (iii). By using Lemma 18, we have for the functional $J$ :

$$
\begin{aligned}
& J\left(\sum_{i=1}^{p} \alpha_{i} \hat{\delta}\left(x_{i}, \lambda\right)\right) \\
& \leq \frac{\left\{\sum_{i=1}^{p} \alpha_{i}^{2} \int_{M} L \hat{\delta}\left(x_{i}, \lambda\right) \hat{\delta}\left(x_{i}, \lambda\right)+2 \sum_{i<j} \alpha_{i} \alpha_{j} \int_{M} L \hat{\delta}\left(x_{i}, \lambda\right) \hat{\delta}\left(x_{j}, \lambda\right)\right\}^{1+\frac{1}{n}}}{\int_{M} \sum_{i=1}^{p} \alpha_{i}^{2+\frac{2}{n}} \hat{\delta}\left(x_{i}, \lambda\right)^{2+\frac{2}{n}}+\frac{\left(2+\frac{2}{n}\right) \gamma}{2} \sum_{i \neq j} \alpha_{i}^{1+\frac{2}{n}} \alpha_{j} \int_{M} \hat{\delta}\left(x_{i}, \lambda\right)^{1+\frac{2}{n}} \hat{\delta}\left(x_{j}, \lambda\right)} .
\end{aligned}
$$

We derive from Lemmas 3 and 4 that

$$
\begin{aligned}
& J\left(\sum_{i=1}^{p} \alpha_{i} \hat{\delta}\left(x_{i}, \lambda\right)\right) \\
& \leq \frac{\left\{\sum_{i=1}^{p} \alpha_{i}^{2}\left(S^{n}+O\left(\frac{1}{\lambda^{2 n}}\right)\right)+\left(1+\frac{\gamma}{2}\right) \sum_{i \neq j} \alpha_{i} \alpha_{j} \int_{M} \hat{\delta}\left(x_{i}, \lambda\right)^{1+\frac{2}{n}} \hat{\delta}\left(x_{j}, \lambda\right)\right\}^{1+\frac{1}{n}}}{\sum_{i=1}^{p} \alpha_{i}^{2+\frac{2}{n}}\left(S^{n}+O\left(\frac{1}{\lambda^{2 n}}\right)\right)+\left(1+\frac{1}{n}\right) \gamma \sum_{i \neq j} \alpha_{i}^{1+\frac{2}{n}} \alpha_{j} \int_{M} \hat{\delta}\left(x_{i}, \lambda\right)^{1+\frac{2}{n}} \hat{\delta}\left(x_{j}, \lambda\right)} .
\end{aligned}
$$

Thus

$$
\begin{aligned}
& J\left(\sum_{i=1}^{p} \alpha_{i} \hat{\delta}\left(x_{i}, \lambda\right)\right) \\
& \leq \frac{\left(\sum_{i=1}^{p} \alpha_{i}^{2}\right)^{1+\frac{1}{n}} S\left\{1+O\left(\frac{1}{\lambda^{2 n}}\right)+\frac{2\left(1+\frac{\gamma}{2}\right)}{\left(\sum_{i=1}^{p} \alpha_{i}^{2}\right) S^{n}} \sum_{i<j} \alpha_{i} \alpha_{j} \int_{M} \hat{\delta}\left(x_{i}, \lambda\right)^{1+\frac{2}{n}} \hat{\delta}\left(x_{j}, \lambda\right)\right\}^{1+\frac{1}{n}}}{\sum_{i=1}^{p} \alpha_{i}^{2+\frac{2}{n}}\left\{1+O\left(\frac{1}{\lambda^{2 n}}\right)+\frac{\left(1+\frac{1}{n}\right) \gamma \sum_{i \neq j} \alpha_{i}^{1+\frac{2}{n}} \int_{M} \hat{\delta}\left(x_{i} \lambda\right) \hat{\delta}\left(x_{j} \lambda\right)}{\left(\sum_{i=1}^{p} \alpha_{i}^{2+\frac{2}{n}}\right) S^{n}}\right\}} .
\end{aligned}
$$

Let us assume that

$$
\sum_{i \neq j} \int_{M} \hat{\delta}\left(x_{i}, \lambda\right)^{1+\frac{2}{n}} \hat{\delta}\left(x_{j}, \lambda\right) \theta \wedge d \theta^{n}<\epsilon_{1},
$$

if $\epsilon_{1}$ is chosen small enough; then, for $\lambda$ large enough, we have:

$$
\begin{aligned}
J & \left(\sum_{i=1}^{p} \alpha_{i} \hat{\delta}\left(x_{i}, \lambda\right)\right) \\
\leq & \frac{\left(\sum_{i=1}^{p} \alpha_{i}^{2}\right)^{1+\frac{1}{n}}}{\sum_{i=1}^{p} \alpha_{i}^{2+\frac{2}{n}}} S\left\{1+O\left(\frac{1}{\lambda^{2 n}}\right)\right. \\
& +\frac{\left(1+\frac{\gamma}{2}\right)\left(1+\frac{1}{n}\right) \sum_{i \neq j} \alpha_{i} \alpha_{j} \int_{M} \hat{\delta}\left(x_{i} \lambda\right)^{1+\frac{2}{n}} \delta\left(x_{j}, \lambda\right)}{\left(\sum_{i=1}^{p} \alpha_{i}^{2}\right) S^{n}} \\
& \left.-\frac{\left(1+\frac{1}{n}\right) \gamma \sum_{i \neq j} \alpha_{i}^{1+\frac{2}{n}} \alpha_{j} \int_{M} \hat{\delta}\left(x_{i}, \lambda\right)^{1+\frac{2}{n}} \hat{\delta}\left(x_{j}, \lambda\right)}{\left(\sum_{i=1}^{p} \alpha_{i}^{2+\frac{2}{n}}\right) S^{n}}\right\}
\end{aligned}
$$


and finally it follows that:

$$
\begin{aligned}
& J\left(\sum_{i=1}^{p} \alpha_{i} \hat{\delta}\left(x_{i}, \lambda\right)\right) \\
& \leq \frac{\left(\sum_{i=1}^{p} \alpha_{i}^{2}\right)^{1+\frac{1}{n}}}{\sum_{i=1}^{p} \alpha_{i}^{2+\frac{2}{n}}} S\left\{1+O\left(\frac{1}{\lambda^{2 n}}\right)+\frac{1}{C} \sum_{i \neq j} \int_{M} \hat{\delta}\left(x_{i}, \lambda\right)^{1+\frac{2}{n}} \hat{\delta}\left(x_{j}, \lambda\right)\right\} .
\end{aligned}
$$

Hence (iii).

Proof of (ii). Let us now assume that there exists $0<\beta<1$ such that, $\frac{\alpha_{i}}{\alpha_{j}} \geq \beta$ for any $(i, j)$.

Our aim is to find an upperbound of:

$$
S^{-n}\left(\left(1+\frac{\gamma}{2}\right)\left(1+\frac{1}{n}\right) \frac{\alpha_{i} \alpha_{j}}{\sum_{i=1}^{p} \alpha_{i}^{2}}-\gamma\left(1+\frac{1}{n}\right) \frac{\alpha_{i}^{1+\frac{2}{n}} \alpha_{j}}{\sum_{i=1}^{p} \alpha_{i}^{2+\frac{2}{n}}}\right) .
$$

Then

and

$$
\frac{\left(1+\frac{\gamma}{2}\right)\left(1+\frac{1}{n}\right) \alpha_{i} \alpha_{j}}{\sum_{i=1}^{p} \alpha_{i}^{2}} \leq \frac{\left(1+\frac{\gamma}{2}\right)\left(1+\frac{1}{n}\right)}{p \beta^{2}}
$$

$$
\frac{\left(1+\frac{1}{n}\right) \gamma \alpha_{i}^{1+\frac{2}{n}} \alpha_{j}}{\sum_{i=1}^{p} \alpha_{i}^{2+\frac{2}{n}}} \geq \frac{\beta^{2+\frac{2}{n}}}{p}\left(1+\frac{1}{n}\right) \gamma
$$

Assume now that we choose $0<\beta<1$ so that

$$
\frac{\left(1+\frac{\gamma}{2}\right)\left(1+\frac{1}{n}\right)}{\beta^{2}}<\beta^{2+\frac{2}{n}}\left(1+\frac{1}{n}\right) \gamma
$$

let

$$
\delta=\left(\beta^{2+\frac{2}{n}}\left(1+\frac{1}{n}\right) \gamma-\frac{\left(1+\frac{\gamma}{2}\right)\left(1+\frac{1}{n}\right)}{\beta^{2}}\right) S^{-n}
$$

we then derive

$$
\begin{aligned}
J\left(\sum_{i=1}^{p} \alpha_{i} \hat{\delta}\left(x_{i}, \lambda\right)\right) \\
\leq \frac{\left(\sum_{i=1}^{p} \alpha_{i}^{2}\right)^{1+\frac{1}{n}}}{\sum_{i=1}^{p} \alpha_{i}^{2+\frac{2}{n}}} S\left\{1+O\left(\frac{1}{\lambda^{2 n}}\right)\right. \\
\left.-\frac{\delta}{p} \sum_{i \neq j} \int_{M} \hat{\delta}\left(x_{i}, \lambda\right)^{1+\frac{2}{n}} \hat{\delta}\left(x_{j}, \lambda\right)\right\}
\end{aligned}
$$

and (ii) follows by using (v) of Lemma 4. 
Lemma 17 implies the following proposition:

Proposition 19. There exist an integer $p_{0}>0$, and a positive real number $\lambda_{0}$, such that for any $\left(\alpha_{1}, \ldots, \alpha_{p}\right)$ satisfying $\alpha_{i} \geq 0, \sum_{i=1}^{p} \alpha_{i}=1, \quad p \geq p_{0}$, for any $\left(x_{1}, \ldots, x_{p}\right) \in M^{p}$ and for any $\lambda \geq \lambda_{0}$ we have:

$$
J\left(\sum_{i=1}^{p} \alpha_{i} \hat{\delta}\left(x_{i}, \lambda\right)\right) \leq p^{\frac{1}{n}} S .
$$

Proof. This proposition is a consequence of the inequalities (i), (ii) and (iii) of Lemma 17.

Let us assume first that there is a couple of indexes $\left(i_{0}, j_{0}\right)$ such that $\frac{\alpha_{i_{0}}}{\alpha_{j_{0}}} \leq \beta$, we consider then $\lambda \geq \lambda_{0}=\lambda(p, \epsilon)$ and we have from (i) of Lemma 17:

$$
J\left(\sum_{i=1}^{p} \alpha_{i} \hat{\delta}\left(x_{i}, \lambda\right)\right) \leq p^{\frac{1}{n}} S
$$

if

$$
\sum_{i \neq j} \int_{M} \hat{\delta}\left(x_{i}, \lambda\right)^{1+\frac{2}{n}} \hat{\delta}\left(x_{j}, \lambda\right) \theta \wedge d \theta^{n} \geq \epsilon
$$

Otherwise, if

$$
\sum_{i \neq j} \int_{M} \hat{\delta}\left(x_{i}, \lambda\right)^{1+\frac{2}{n}} \hat{\delta}\left(x_{j}, \lambda\right) \theta \wedge d \theta^{n}<\epsilon,
$$

we apply the inequality (iii) of the lemma by choosing $\epsilon=\epsilon(p, \beta)$ such that

$$
\frac{\left(\sum_{i=1}^{p} \alpha_{i}^{2}\right)^{1+\frac{1}{n}}}{\sum_{i=1}^{p} \alpha_{i}^{2+\frac{2}{n}}}\left(1+O\left(\frac{1}{\lambda^{2 n}}\right)+\frac{\epsilon}{C}\right)<p^{\frac{1}{n}},
$$

and Proposition 19 is established with $p_{0}=p$.

Let us now assume that for all indices $i, j(i \neq j), \frac{\alpha_{i}}{\alpha_{j}}>\beta$.

Then, the inequalities (i) and (ii) of Lemma 17 still hold with $\beta$ in (ii) independent of $p$. In (ii), we choose $p_{0}$ such that $\frac{p_{0}+1}{C^{2}}>1$. It follows that for all $p \geq p_{0}$ we have:

$$
J\left(\sum_{i=1}^{p} \alpha_{i} \hat{\delta}\left(x_{i}, \lambda\right)\right) \leq \frac{\left(\sum_{i=1}^{p} \alpha_{i}^{2}\right)^{1+\frac{1}{n}}}{\sum_{i=1}^{p} \alpha_{i}^{2+\frac{2}{n}}} S \leq p^{\frac{1}{n}} S .
$$

Proposition 19 follows.

We introduce the following notations: 
For any $p \geq 1$ and $\lambda>0$ let

$$
\begin{aligned}
B_{p}=B_{p}(M)=\left\{\sum_{i=1}^{p} \alpha_{i} \delta_{x_{i}}, \alpha_{i} \geq 0, \sum_{i=1}^{p} \alpha_{i}=1,\right. & \left.x_{i} \in M\right\} \\
& \text { and } B_{0}=B_{0}(M)=\emptyset
\end{aligned}
$$

(where $\delta_{x_{i}}$ is the Dirac mass in $x_{i}$ )

$$
W_{p}=\left\{u \in \sum_{+}, \text {such that } J(u)<(p+1)^{\frac{1}{n}} S\right\}
$$

and $f_{p}(\lambda)$ the map of $B_{p}(M)$ in $\sum_{+}$defined by

$$
f_{p}(\lambda)\left(\sum_{i=1}^{p} \alpha_{i} \delta_{x_{i}}\right)=\frac{\sum_{i=1}^{p} \alpha_{i} \hat{\delta}\left(x_{i}, \lambda\right)}{\left\|\sum_{i=1}^{p} \alpha_{i} \hat{\delta}\left(x_{i}, \lambda\right)\right\|_{H}} .
$$

Clearly we have

$$
B_{p-1}(M) \subset B_{p}(M)
$$

and

$$
W_{p-1} \subset W_{p} .
$$

With these notations we have the following proposition:

Proposition 20. (i) For any integer $p \geq 1$, there exists a real $\lambda_{p}>0$, such that

$$
f_{p}(\lambda): B_{p}(M) \longrightarrow W_{p}
$$

for any $\lambda \geq \lambda_{p}$.

(ii) There exists an integer $p_{0} \geq 1$, such that for any integer $p \geq p_{0}$ and for any $\lambda \geq \lambda_{p_{0}}$, the map of pairs:

$$
f_{p}(\lambda):\left(B_{p}, B_{p-1}\right) \longrightarrow\left(W_{p}, W_{p-1}\right)
$$

satisfies $f_{p^{*}}(\lambda) \equiv 0$ where

$$
f_{p^{*}}(\lambda): H_{*}\left(B_{p}, B_{p-1}\right) \longrightarrow H_{*}\left(W_{p}, W_{p-1}\right) .
$$

$H_{*}(\bullet)$ is the homology group with $\mathbb{Z} / 2$ coefficients of $\bullet$.

Proof. (i) is a direct consequence of inequalities (i), (ii) and (iii) of Lemma 17, since we have:

$$
\begin{aligned}
J\left(f_{p}(\lambda)\left(\sum_{i=1}^{p} \alpha_{i} \delta_{x_{i}}\right)\right) & =J\left(\frac{\sum_{i=1}^{p} \alpha_{i} \hat{\delta}\left(x_{i}, \lambda\right)}{\left\|\sum_{i=1}^{p} \alpha_{i} \hat{\delta}\left(x_{i}, \lambda\right)\right\|_{H}}\right) \\
& =J\left(\sum_{i=1}^{p} \alpha_{i} \hat{\delta}\left(x_{i}, \lambda\right)\right) .
\end{aligned}
$$

(ii) follows from Proposition 19. 


\section{Topological arguments.}

Proof of Theorem 1.

Arguing by contradiction, we will assume that $\bar{u} \equiv 0$ (see Section 4), that is the weak limit of the (P.S) sequences are zero; otherwise, our problem would be solved, since we would have found a solution.

We now turn to deformation and topological arguments. We start with:

Lemma 21 ([3, Proposition 5.11]). For any $\epsilon>0$, the pair $\left(W_{p}, W_{p-1}\right)$ retracts by deformation on the pair $\left(W_{p-1} \cup A_{p}, W_{p-1}\right)$, where $A_{p} \subset V(p, \epsilon)$.

Throughout this section we shall denote by $H_{*}(M)$, respectively $H^{*}(M)$, the homology, respectively the cohomology group, with $\mathbb{Z} / 2$ coefficients, and $\omega_{p}$ the orientation class (modulo 2 ), of the pair $\left(B_{p}(M), B_{p-1}(M)\right)$.

We are going to prove by induction on $p$, that:

Proposition 22. For every $p \in \mathbb{N}^{*}, f_{p^{*}}(\lambda)\left(\omega_{p}\right) \neq 0$.

This proposition is in contradiction with Proposition 20. Therefore (1) has a solution and hence Theorem 1 is established.

In order to prove Proposition 22, we introduce the following notations and constructions, which are extracted from [4] and [3], and written for the sake of completeness.

We provide $B_{p}(M)$ with the weak topology of measures. $B_{p}(M)$ with its topology can also be viewed as the quotient space $M^{p} \underset{\sigma_{p}}{\times} \Delta_{p-1}$, where

$$
\Delta_{p-1}=\left\{\left(\alpha_{1}, \ldots, \alpha_{p}\right) \mid \alpha_{i} \in[0,1] \forall i, \sum_{i=1}^{p} \alpha_{i}=1\right\}
$$

and $\sigma_{p}$ the group of permutations of $\{1, \ldots, p\}$.

Let

$$
F_{p}=\left\{\left(x_{1}, \ldots, x_{p}\right) \in M^{p} \mid \exists(i, j) \in\{1, \ldots, p\}^{2} \text { with } x_{i}=x_{j} \text { and } i \neq j\right\}
$$

and let $T_{p}$ be a tubular neighborhood of $F_{p}$ in $M^{p}$ which is invariant by $\sigma_{p}$, and such that $M_{0}^{p}=M^{p} \backslash T_{p}$ is a manifold with boundary $\partial M_{0}^{p}$ (the existence of $T_{p}$ is derived in the book by G. Bredon [6]). In order to construct such a $T_{p}$, one can proceed as in Appendix $\mathrm{C}$ of [4].

$M_{0}^{p} \underset{\sigma_{p}}{\times} \Delta_{p-1}$ can be viewed as the quotient space $M_{0}^{p} \underset{\sigma_{1} \times \sigma_{p-1}}{\times} \Delta_{p-1}$ by the

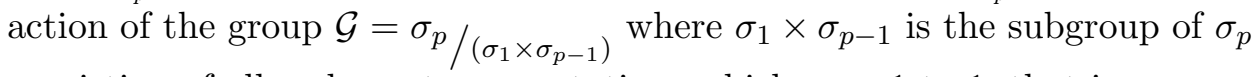
consisting of all $p$ elements permutations which map 1 to 1 , that is

$$
M_{0}^{p} \underset{\sigma_{p}}{\times} \Delta_{p-1} \simeq\left(M_{0}^{p} \underset{\sigma_{1} \times \sigma_{p-1}}{\times} \Delta_{p-1}\right) / \mathcal{G} .
$$


We have the following diagram:

$$
\begin{aligned}
& M_{0}^{p} \underset{\sigma_{1} \times \sigma_{p-1}}{\times} \Delta_{p-1} \stackrel{p_{1}}{\longrightarrow} M \\
& \quad q \\
& M_{0}^{p} \underset{\sigma_{p}}{\downarrow} \Delta_{p-1} .
\end{aligned}
$$

The projection q defines a covering with associated group $\mathcal{G}$. We then have (see [3]) a well-defined homological transfer maps, which are the transformed functions of $q^{*}$ and $q_{*}$ via the Poincaré duality:

$$
\begin{aligned}
& \operatorname{tr}_{*}: H^{*}\left(M_{0}^{p} \underset{\sigma_{1} \times \sigma_{p-1}}{\times} \Delta_{p-1}\right) \longrightarrow H^{*}\left(M_{0}^{p} \underset{\sigma_{p}}{\times} \Delta_{p-1}\right) \\
& \operatorname{tr}_{*}: H_{*}\left(M_{0}^{p} \underset{\sigma_{p}}{\times} \Delta_{p-1}\right) \longrightarrow H_{*}\left(M_{0}^{p} \underset{\sigma_{1} \times \sigma_{p-1}}{\left.\times \underset{\Delta_{p-1}}{\times} \Delta_{p-1}\right) .} .\right.
\end{aligned}
$$

Definition 23. Let $O_{M}^{*}$ be the $2 n+1$ cohomological class of $M_{0}^{p} \underset{\sigma_{p}}{\times} \Delta_{p-1}$ defined by $O_{M}^{*}=t r^{*} \circ p_{1}^{*}\left([M]^{*}\right)$ where $[M]^{*}$ is the cohomological orientation class of $M$.

For a fixed real $0<\eta<1$, let $V\left(B_{p-1}\right)$ denote the neighborhood of $B_{p-1}(M)$ in $B_{p}(M)$ defined by

$$
V\left(B_{p-1}\right)=B_{p-1}(M) \cup\left(B_{p}(M) \backslash h^{-1}\left(M_{0}^{p} \underset{\sigma_{p}}{\times} \Delta_{p-1}^{\eta}\right)\right)
$$

where $h^{-1}$ is the natural map from $M_{0}^{p} \times \Delta_{p-1}^{\eta}$ into $B_{p}(M)$ and

$$
\Delta_{p-1}^{\eta}=\left\{\left(\alpha_{1}, \ldots, \alpha_{p}\right) \in[0,1]^{p}, \text { such that } \sum_{i=1}^{p} \alpha_{i}=1 \text { and } \alpha_{i} \geq \eta\right\} .
$$

We have the following result:

Lemma 24 ([4], [3]).

(i) $B_{p-1}(M)$ is a retract by deformation of $V\left(B_{p-1}\right)$.

(ii) $f_{p}(\lambda)\left(V\left(B_{p-1}\right)\right) \subset W_{p-1}$.

(iii) $f_{p}(\lambda)\left(B_{p} \backslash V\left(B_{p-1}\right)\right) \subset A_{p}$, where $A_{p}$ is a subset of $V(p, \epsilon)$.

Using Lemma 24, Proposition 22 reduces to: $f_{p *}(\lambda)\left(\omega_{p}\right) \not \equiv 0$ for every $p \in \mathbb{N}^{*}$, where $f_{p}(\lambda)$ is now the map:

$$
f_{p}(\lambda):\left(B_{p} \backslash V\left(B_{p-1}\right), \partial V\left(B_{p-1}\right)\right) \longrightarrow\left(W_{p-1} \cup A_{p}, W_{p-1}\right) .
$$

We have the following commutative diagram: 


$$
\begin{aligned}
& \left(B_{p} \backslash V\left(B_{p-1}\right), \partial V\left(B_{p-1}\right)\right) \stackrel{f_{p}(\lambda)}{\longrightarrow}\left(W_{p-1} \cup A_{p}, W_{p-1}\right) \\
& \gamma \downarrow r_{1} \\
& \left(M_{0}^{p} \underset{\sigma_{p}}{\times} \Delta_{p-1}^{\eta}, \quad \partial\left(M_{0}^{p} \underset{\sigma_{p}}{\times} \Delta_{p-1}^{\eta}\right)\right)
\end{aligned}
$$

$f_{p}(\lambda)$ associates to $\sum_{i=1}^{p} \alpha_{i} \delta_{x_{i}}$ the function

$$
\frac{\sum_{i=1}^{p} \alpha_{i} \hat{\delta}\left(x_{i}, \lambda\right)}{\left\|\sum_{i=1}^{p} \alpha_{i} \hat{\delta}\left(x_{i}, \lambda\right)\right\|_{H}}
$$

$\gamma$ associates to $\sum_{i=1}^{p} \alpha_{i} \delta_{x_{i}}$ the point $\left(x_{1}, \ldots, x_{p}, \alpha_{1}, \ldots, \alpha_{p}\right)$ of $M_{0}^{p} \underset{\sigma_{p}}{\times} \Delta_{p-1}^{\eta}$.

$r_{1}$ is defined through Lemma 7 and associates to

$$
\frac{\sum_{i=1}^{p} \alpha_{i} \hat{\delta}\left(x_{i}, \lambda\right)}{\left\|\sum_{i=1}^{p} \alpha_{i} \hat{\delta}\left(x_{i}, \lambda\right)\right\|_{H}}
$$

the corresponding point of $M_{0}^{p} \underset{\sigma_{p}}{\times} \Delta_{p-1}^{\eta}$.

The pair $\left(B_{p}, B_{p-1}\right)$ retracts by deformation on the pair $\left(B_{p}, V\left(B_{p-1}\right)\right)$. Thus

$$
H_{*}\left(B_{p}, B_{p-1}\right)=H_{*}\left(B_{p}, V\left(B_{p-1}\right)\right)
$$

and

$$
H^{*}\left(B_{p}, B_{p-1}\right)=H^{*}\left(B_{p}, V\left(B_{p-1}\right)\right) .
$$

By excision we have:

$$
\left\{\begin{array}{l}
H_{*}\left(B_{p}, V\left(B_{p-1}\right)\right)=H_{*}\left(B_{p} \backslash V\left(B_{p-1}\right), \partial V\left(B_{p-1}\right)\right) \\
H^{*}\left(B_{p}, V\left(B_{p-1}\right)\right)=H^{*}\left(B_{p} \backslash V\left(B_{p-1}\right), \partial V\left(B_{p-1}\right)\right) .
\end{array}\right.
$$

We then have:

$$
\begin{aligned}
& H^{*}\left(B_{p} \backslash V\left(B_{p-1}\right), \partial V\left(B_{p-1}\right)\right) \stackrel{f_{p}^{*}(\lambda)}{\gamma^{*} \uparrow} H^{*}\left(W_{p-1} \cup A_{p}, W_{p-1}\right) \\
& H^{*}\left(M_{0}^{*} \underset{\sigma_{p}}{\times} \Delta_{p-1}^{\eta}, \partial\left(M_{0}^{p} \underset{\sigma_{p}}{\times} \Delta_{p-1}^{\eta}\right)\right) .
\end{aligned}
$$

Since

$$
f_{p}^{*}(\lambda) \circ r_{1}^{*}=\gamma^{*}
$$


we have

$$
\left(f_{p}^{*}(\lambda) \circ r_{1}^{*}\right)\left(O_{M}^{*}\right)=\gamma^{*}\left(O_{M}^{*}\right)
$$

By naturality of the cap product $\cap($ see $[7,12.6$, p. 239]) we have:

$$
r_{1}^{*}\left(O_{M}^{*}\right) \cap\left(f_{p *}(\lambda)\right)\left(\omega_{p}\right)=\left(f_{p *}(\lambda)\right)\left(f_{p}^{*}(\lambda)\left(r_{1}^{*}\left(O_{M}^{*}\right)\right) \cap \omega_{p}\right) .
$$

Indeed,

$$
\omega_{p} \in H_{(2 n+1) p+p-1}\left(B_{p}, B_{p-1}\right),
$$

thus

$$
f_{p *}(\lambda)\left(\omega_{p}\right) \in H_{(2 n+1) p+p-1}\left(W_{p-1} \cup A_{p}, W_{p-1}\right)
$$

and

$$
O_{M}^{*} \in H^{2 n+1}\left(M_{0}^{p} / \sigma_{p}\right)
$$

thus

$$
r_{1}^{*}\left(O_{M}^{*}\right) \in H^{2 n+1}\left(W_{p-1} \cup A_{p}, W_{p-1}\right) .
$$

By excision, we have:

$$
H^{2 n+1}\left(W_{p-1} \cup A_{p}, W_{p-1}\right) \simeq H^{2 n+1}\left(A_{p}, A_{p} \cap W_{p-1}\right) .
$$

We then derive:

$$
r_{1}^{*}\left(O_{M}^{*}\right) \cap f_{p *}(\lambda)\left(\omega_{p}\right) \in H_{(2 n+1)(p-1)+p-1}\left(A_{p}, A_{p} \cap W_{p-1}\right)
$$

and using (6.4), we obtain

$$
\begin{aligned}
f_{p *}(\lambda)\left(\gamma^{*}\left(O_{M}^{*}\right) \cap \omega_{p}\right) & =f_{p *}(\lambda)\left(f_{p}^{*}(\lambda)\left(r_{1}^{*}\left(O_{M}^{*}\right)\right) \cap \omega_{p}\right) \\
& =r_{1}^{*}\left(O_{M}^{*}\right) \cap f_{p *}(\lambda)\left(\omega_{p}\right) .
\end{aligned}
$$

On the other hand we have the following commutative diagram

$$
\begin{array}{cc}
H_{*}\left(B_{p}, B_{p-1}\right) \stackrel{f_{p *}(\lambda)}{\longrightarrow} H_{*}\left(W_{p-1} \cup A_{p}, W_{p-1}\right) \\
\delta \downarrow & \downarrow \delta_{1} \\
H_{*-1}\left(B_{p-1}\right) \stackrel{f_{(p-1) *}(\lambda)}{\longrightarrow} H_{*-1}\left(W_{p-1}\right) \\
j_{*} \downarrow & \downarrow j_{1 *} \\
H_{*-1}\left(B_{p-1}, B_{p-2}\right) \stackrel{f_{(p-1) *}(\lambda)}{\longrightarrow} H_{*-1}\left(W_{p-1}, W_{p-2}\right)
\end{array}
$$

where the vertical maps are the exact sequences of the appropriate pairs.

Using these notations, we have the following lemma:

Lemma 25 ([4, Section 8, Lemma 16]). We have

$$
j_{*} \circ \delta\left(\gamma^{*}\left(O_{M}^{*}\right) \cap \omega_{p}\right)=\omega_{p-1} .
$$


Using (6.4) and what follows as well as (6.5), we obtain:

$$
\begin{aligned}
j_{1 *} \circ \delta_{1}\left(r_{1}^{*}\left(O_{M}^{*}\right) \cap f_{p *}\left(\omega_{p}\right)\right) & =j_{1 *} \circ \delta_{1}\left(f_{p *}\left(\gamma^{*}\left(O_{M}^{*}\right) \cap \omega_{p}\right)\right) \\
& =f_{(p-1) *} \circ j_{*} \circ \delta\left(\gamma^{*}\left(O_{M}^{*}\right) \cap \omega_{p}\right) \\
& =f_{(p-1) *}\left(\omega_{p-1}\right) .
\end{aligned}
$$

In Appendix B, we prove the following result:

Lemma 26. If there is no solution of (1), then for $\epsilon>0$ small enough we have:

is a retract of $M$.

$$
J_{S+\epsilon}=\left\{u \in \sum_{+} / J(u) \leq S+\epsilon\right\}
$$

Proof of Proposition 22. Since $J_{S+\epsilon}$, for $\epsilon>0$ small enough, satisfies $J_{S+\epsilon} \subset V(1, \delta)$, where $\delta \rightarrow 0$ if $\epsilon \rightarrow 0$. We derive, using Lemma 7, that $f_{1 *}(\lambda)\left(\omega_{1}\right) \neq 0$. Suppose now, that at the order $(p-1)$ we have $\left(f_{(p-1) *}(\lambda)\right)\left(\omega_{p-1}\right) \neq 0$. Then, we derive from (6.6) that

$$
r_{1}^{*}\left(O_{M}^{*}\right) \cap\left(f_{p *}(\lambda)\left(\omega_{p}\right)\right) \neq 0,
$$

that is

$$
f_{p *}(\lambda)\left(\omega_{p}\right) \neq 0,
$$

which ends the proof of Proposition 22.

Proof of Lemma 25. Let $\omega_{p}$ be the homological orientation class of the pair $\left(B_{p} \backslash V\left(B_{p-1}\right), \partial V\left(B_{p-1}\right)\right)$ which is identified, via $h$, with the pair $\left(M_{0}^{p} \underset{\sigma_{p}}{\times} \Delta_{p-1}^{\eta}, \partial\left(M_{0}^{p} \times \underset{\sigma_{p}}{\times} \Delta_{p-1}^{\eta}\right)\right)$.

Since we have a covering

$$
\begin{aligned}
\left(M_{0}^{p} \underset{\sigma_{1} \times \sigma_{p-1}}{\times} \Delta_{p-1}^{\eta}, \partial\left(M_{0}^{p} \underset{\sigma_{1} \times \sigma_{p-1}}{\times} \Delta_{p-1}^{\eta}\right)\right) & \\
& \longrightarrow\left(M_{0}^{p} \underset{\sigma_{p}}{\times} \Delta_{p-1}^{\eta}, \partial\left(M_{0}^{p} \underset{\sigma_{p}}{\times} \Delta_{p-1}^{\eta}\right)\right)
\end{aligned}
$$

we can apply the following formula (see [7], p. 314):

$$
q_{*}\left(u^{*} \cap t r_{*}(\omega)\right)=t r^{*}\left(u^{*}\right) \cap \omega
$$

where

$$
\omega \in H_{*}\left(M_{0}^{p} \underset{\sigma_{p}}{\times} \Delta_{p-1}^{\eta}, \partial\left(M_{0}^{p} \underset{\sigma_{p}}{\times} \Delta_{p-1}^{\eta}\right)\right)
$$

and

$$
u^{*} \in H^{*}\left(M_{0}^{p} \underset{\sigma_{1} \times \sigma_{p-1}}{\times} \Delta_{p-1}^{\eta}, \partial\left(M_{0}^{p} \underset{\sigma_{1} \times \sigma_{p-1}}{\times} \Delta_{p-1}^{\eta}\right)\right) .
$$

The formula (6.7) can be understood in an abstract way if we can understand the cap product: To do this we must introduce elaborated notions, such as 
for example the applications of Eilenberg-Zilber. However there is a context where this formula can be understood easily: We will consider an example in Appendix C, the case where $M=S^{k}, k \geq 2$ and $p=2$.

We replace in (6.7) $\omega$ by $\gamma_{*}\left(\omega_{p}\right)$ and $u^{*}$ by $p_{1}^{*}[M]^{*}$, we have $t r^{*}\left(u^{*}\right)=O_{M}^{*}$ and we derive that

$$
O_{M}^{*} \cap \gamma_{*}\left(\omega_{p}\right)=q_{*}\left(p_{1}^{*}[M]^{*} \cap \operatorname{tr}_{*}\left(\gamma_{*}\left(\omega_{p}\right)\right)\right) .
$$

Since we have a covering:

$$
\operatorname{tr}_{*}\left(\gamma_{*}\left(\omega_{p}\right)\right)=\widetilde{\omega}_{p}
$$

where $\widetilde{\omega}_{p}$ is the homological orientation class of

$$
\left(M_{0}^{p} \underset{\sigma_{1} \times \sigma_{p-1}}{\times} \Delta_{p-1}^{\eta}, \partial\left(M_{0}^{p} \underset{\sigma_{1} \times \sigma_{p-1}}{\times} \Delta_{p-1}^{\eta}\right)\right) .
$$

Let us denote by

$$
p_{1}: M_{0}^{p} \underset{\sigma_{1} \times \sigma_{p-1}}{\times} \Delta_{p-1}^{\eta} \longrightarrow M
$$

the projection on the first factor, and $M_{\xi}=p_{1}^{-1}(\xi)$ the fiber over $\xi \in M$ :

$$
\begin{gathered}
M_{\xi}=\left\{\left(\xi, x_{\sigma(2)}, \ldots, x_{\sigma(p)}, \alpha_{1}, \alpha_{\sigma(2)}, \ldots, \alpha_{\sigma(p)}\right) \in M_{0}^{p} \underset{\sigma_{1} \times \sigma_{p-1}}{\times} \Delta_{p-1}^{\eta}\right. \\
\text { such that: } \\
\alpha_{1}+\sum_{i=2}^{p} \alpha_{\sigma(i)}=1, \\
\alpha_{i} \geq \eta \text { for } i \in\{1, \ldots, p\}, \\
\widetilde{d}\left(\xi, x_{\sigma(i)}\right) \geq \eta_{1} \text { for } i \in\{2, \ldots, p\}, \\
\text { and } \left.\widetilde{d}\left(x_{\sigma(i)}, x_{\sigma(j)}\right) \geq \eta_{1} \text { for } i, j \in\{2, \ldots, p\} \text { with } i \neq j\right\}
\end{gathered}
$$

( $\eta_{1}$ depends on the tubular neighborhood $\left.T_{p}\right)$.

The projection $p_{1}$ is a fibration of

$$
\left(M_{0}^{p} \underset{\sigma_{1} \times \sigma_{p-1}}{\times} \Delta_{p-1}^{\eta}, \partial\left(M_{0}^{p} \underset{\sigma_{1} \times \sigma_{p-1}}{\times} \Delta_{p-1}^{\eta}\right)\right)
$$

over $M$ with fiber $\left(M_{\xi}, \partial M_{\xi}\right)$, and $M_{\xi}$ is a manifold with boundary, of dimension

$$
(2 n+1)(p-1)+p-1 .
$$


Would the fibration be trivial we would then have for any $s$ :

$$
\begin{aligned}
& H^{s}\left(M_{0}^{p} \underset{\sigma_{1} \times \sigma_{p-1}}{\times} \Delta_{p-1}^{\eta}, \partial\left(M_{0}^{p} \underset{\sigma_{1} \times \sigma_{p-1}}{\times} \Delta_{p-1}^{\eta}\right)\right) \\
& =\bigoplus_{i=0}^{i=s} H^{i}(M) \otimes H^{s-i}\left(M_{\xi}, \partial M_{\xi}\right) .
\end{aligned}
$$

The fibration is not trivial. Therefore this result, does not hold. However for the top dimension $s=(2 n+1) p+p-1$, this result will hold using a triangulation of the manifold

$$
\left(M_{0}^{p} \underset{\sigma_{1} \times \sigma_{p-1}}{\times} \Delta_{p-1}^{\eta}, \partial\left(M_{0}^{p} \underset{\sigma_{1} \times \sigma_{p-1}}{\times} \Delta_{p-1}^{\eta}\right)\right)
$$

adapted to the fibration. Thus,

$$
\widetilde{\omega}_{p}=[M]_{*} \otimes \omega_{p-1}^{\prime}
$$

where $\omega_{p-1}^{\prime}$ is the homological orientation class of $\left(M_{\xi}, \partial M_{\xi}\right)$. We have:

$$
p_{1}^{*}\left([M]^{*}\right)=[M]^{*} \otimes 1,
$$

since $p_{1}$ is a fibration, where $1 \in H^{0}\left(M_{\xi}\right) \simeq \mathbb{Z}$.

We derive from (6.8), (6.10) and (6.11) that

$$
p_{1}^{*}\left([M]^{*}\right) \cap \operatorname{tr}_{*}\left(\gamma_{*}\left(\omega_{p}\right)\right)=\omega_{p-1}^{\prime} .
$$

Thus, we obtain

$$
O_{M}^{*} \cap \gamma_{*}\left(\omega_{p}\right)=q_{*}\left(p_{1}^{*}[M]^{*} \cap \operatorname{tr}_{*}\left(\gamma_{*}\left(\omega_{p}\right)\right)\right)=q_{*}\left(\omega_{p-1}^{\prime}\right)
$$

hence,

$$
\gamma^{*}\left(O_{M}^{*}\right) \cap \omega_{p}=\gamma_{*}^{-1} \circ q_{*}\left(\omega_{p-1}^{\prime}\right) .
$$

For a fixed $\xi \in M$, let now:

$$
\begin{aligned}
& \mathcal{C}_{\xi}\left(B_{p-1}\right)=\left\{(1-t) \delta_{\xi}+\alpha_{1} \delta_{x_{1}}+\cdots+\alpha_{p-1} \delta_{x_{p-1}},\right. \\
& \text { with } \sum_{i=1}^{p-1} \alpha_{i}=t \\
& \text { and for } \left.i \in\{1, \ldots, p-1\}, x_{i} \in M, \alpha_{i} \geq 0\right\}
\end{aligned}
$$




$$
\begin{aligned}
& V_{\xi}\left(B_{p-1}\right)=\left\{(1-t) \delta_{\xi}+\alpha_{1} \delta_{x_{1}}+\cdots+\alpha_{p-1} \delta_{x_{p-1}},\right. \\
& \text { with } \sum_{i=1}^{p-1} \alpha_{i}=t \text { and } \exists i \in\{1, \ldots, p-1\} \\
& \text { such that } \widetilde{d}\left(\xi, x_{i}\right) \leq \eta_{1} \text { or } \exists i_{0}, j_{0} \\
& \text { such that } \left.\widetilde{d}\left(x_{i_{0}}, x_{j_{0}}\right) \leq \eta_{1} \text { or } 1-t<\eta \text { or } t<\eta\right\} .
\end{aligned}
$$

The space $B_{p-1}$ is a retract by deformation of $V_{\xi}\left(B_{p-1}\right)$ in $B_{p}$.

We have the following commutative diagram:

$$
\begin{array}{cc}
H_{*}\left(q_{*}\left(M_{\xi}\right), q_{*}\left(\partial M_{\xi}\right)\right) \longrightarrow & H_{*}\left(\mathcal{C}_{\xi}\left(B_{p-1}\right), V_{\xi}\left(B_{p-1}\right)\right) \\
\delta^{\prime} \downarrow & \downarrow \delta^{\prime \prime} \\
H_{*-1}\left(q_{*}\left(\partial M_{\xi}\right)\right) \longrightarrow & H_{*-1}\left(V_{\xi}\left(B_{p-1}\right)\right) \\
& \downarrow l \\
& H_{*-1}\left(B_{p-1}\right)
\end{array}
$$

where $\Gamma$ is the excision isomorphism, $\delta^{\prime}$ and $\delta^{\prime \prime}$ the connecting homomorphisms, $l$ is uniquely defined by

$$
l \circ \delta^{\prime}=\delta^{\prime \prime} \circ \Gamma
$$

By the arguments above, we have:

$$
j_{*} \circ \delta\left(\gamma^{*}\left(O_{M}^{*}\right) \cap \omega_{p}\right)=l \circ \delta^{\prime}\left(q_{*}\left[M_{\xi}, \partial M_{\xi}\right]\right) .
$$

Since $\Gamma$ is an excision isomorphism, we have:

$$
\Gamma \circ \gamma_{*}^{-1}\left(\left[q_{*}\left(M_{\xi}\right), q_{*}\left(\partial M_{\xi}\right)\right]\right)=\left[\mathcal{C}_{\xi}\left(B_{p-1}\right), V_{\xi}\left(B_{p-1}\right)\right]
$$

where $\gamma_{*}^{-1}\left(q_{*}\left(M_{\xi}\right), q_{*}\left(\partial M_{\xi}\right)\right)$ is $\left(\mathcal{C}_{\xi}\left(B_{p-1}\right), V_{\xi}\left(B_{p-1}\right)\right)$ excised of $V_{\xi}\left(B_{p-1}\right)$. But $\mathcal{C}_{\xi}\left(B_{p-1}\right)$ is contractible in $B_{p}$, that is

$$
H_{*}\left(\mathcal{C}_{\xi}\left(B_{p-1}\right)\right)=0
$$


Thus, we have the following exact sequence:

$$
\begin{gathered}
H_{(2 n+1)(p-1)+p-1}\left(\mathcal{C}_{\xi}\left(B_{p-1}\right)\right) \stackrel{\Theta}{\longrightarrow} H_{(2 n+1)(p-1)+p-1}\left(\mathcal{C}_{\xi}\left(B_{p-1}\right), V_{\xi}\left(B_{p-1}\right)\right) \\
\| \\
\qquad \delta^{\prime \prime} \\
H_{(2 n+1)(p-1)+p-2}\left(V_{\xi}\left(B_{p-1}\right)\right) \\
\downarrow \alpha \\
H_{(2 n+1)(p-1)+p-2}\left(\mathcal{C}_{\xi}\left(B_{p-1}\right)\right) \\
\| \\
0
\end{gathered}
$$

that is: $\mathcal{K} e r \delta^{\prime \prime}=\mathcal{I} m \Theta=0$. Thus, $\delta "$ is one to one.

On the other hand, $\mathcal{I} m \delta^{\prime \prime}=\mathcal{K} e r \alpha=H_{*-1}\left(V_{\xi}\left(B_{p-1}\right)\right)$. Thus, $\delta^{\prime \prime}$ is onto. Hence,

$$
\delta^{\prime \prime}\left(\left[\mathcal{C}_{\xi}\left(B_{p-1}\right), V_{\xi}\left(B_{p-1}\right)\right]\right)=\left[B_{p-1}, B_{p-2}\right]
$$

( $\delta^{\prime \prime}$ is an isomorphism), and

$$
l \circ \delta^{\prime}\left(\gamma^{*}\left(O_{M}^{*}\right) \cap \omega_{p}\right)=\omega_{p-1} .
$$

\section{Appendix A.}

\section{Lemma A.1.}

$$
\int_{B(a, \rho)-B\left(a, \frac{\rho}{2}\right)} \delta(a, \lambda)^{2+\frac{2}{n}} \theta_{0} \wedge d \theta_{0}^{n}=O\left(\frac{1}{\lambda^{2 n+2}}\right) .
$$

Proof. Outside of the ball $B\left(a, \frac{\rho}{2}\right), \delta(a, \lambda)$ is $O\left(\frac{1}{\lambda^{n}}\right)$, Lemma A.1 follows.

\section{Lemma A.2.}

$$
\int_{B(0, \rho)} \delta(a, \lambda)^{2+\frac{2}{n}} \theta_{0} \wedge d \theta_{0}^{n}=\int_{B(0, \lambda \rho)} \delta(a, 1)^{2+\frac{2}{n}} \theta_{0} \wedge d \theta_{0}^{n}
$$

Proof. We consider the dilation on the Heisenberg group $\mathbb{H}^{n}$ :

$$
\lambda(z, t)=\left(\lambda z, \lambda^{2} t\right) .
$$

We have

$$
\begin{aligned}
& \int_{B(0, \rho)}\left(\frac{\lambda^{n}}{\left|1+i \lambda^{2}\left(t+i|z|^{2}\right)\right|^{n}}\right)^{2+\frac{2}{n}} \theta_{0} \wedge d \theta_{0}^{n} \\
& =\int_{B(0, \rho)} \frac{\lambda^{2 n+2}}{\left|1+i \lambda^{2}\left(t+i|z|^{2}\right)\right|^{2 n+2}} \theta_{0} \wedge d \theta_{0}^{n} .
\end{aligned}
$$

We consider the following change of variables $(Z, T)=\lambda(z, t)$. 
We obtain:

$$
\begin{aligned}
& \int_{B(0, \rho)} \frac{\lambda^{2 n+2}}{\left|1+i \lambda^{2}\left(t+i|z|^{2}\right)\right|^{2 n+2}} \theta_{0} \wedge d \theta_{0}^{n} \\
& =\int_{B(0, \rho)} \frac{\lambda^{2 n+2}}{\left|1+i\left(T+i|Z|^{2}\right)\right|^{2 n+2}} \lambda^{-(2 n+2)} \theta_{0} \wedge d \theta_{0}^{n} \\
& =\int_{B(0, \lambda \rho)} \frac{1}{\left|1+i\left(T+i|Z|^{2}\right)\right|^{2 n+2}} \theta_{0} \wedge d \theta_{0}^{n} .
\end{aligned}
$$

We now have:

\section{Lemma A.3.}

$\frac{1}{\lambda^{n}} \int_{B\left(x_{0}, \rho\right)} \delta\left(x_{0}, \lambda\right)^{\frac{2}{n}} \delta\left(x_{1}, \lambda\right) \theta_{0} \wedge d \theta_{0}^{n}=o\left(\int_{\mathbb{H}^{n}} \delta\left(x_{0}, \lambda\right)^{1+\frac{2}{n}} \delta\left(x_{1}, \lambda\right) \theta_{0} \wedge d \theta_{0}^{n}\right)$.

Proof. Let $\epsilon>0$ be given, and

$$
A_{\epsilon}=\left\{x \in B\left(x_{0}, \rho\right), \text { such that } \delta\left(x_{0}, \lambda\right)>\frac{1}{\epsilon \lambda^{n}}\right\} .
$$

We have

$$
\begin{aligned}
& \frac{1}{\lambda^{n}} \int_{B\left(x_{0}, \rho\right)} \delta\left(x_{0}, \lambda\right)^{\frac{2}{n}} \delta\left(x_{1}, \lambda\right) \theta_{0} \wedge d \theta_{0}^{n} \\
& \leq \epsilon \int_{A_{\epsilon}} \delta\left(x_{0}, \lambda\right)^{1+\frac{2}{n}} \delta\left(x_{1}, \lambda\right) \theta_{0} \wedge d \theta_{0}^{n} \\
& \quad+\int_{\mathcal{C}_{A_{\epsilon}}} \frac{\delta\left(x_{0}, \lambda\right)^{\frac{2}{n}} \delta\left(x_{1}, \lambda\right)}{\lambda^{n}} \theta_{0} \wedge d \theta_{0}^{n} \\
& \leq \epsilon \int_{A_{\epsilon}} \delta\left(x_{0}, \lambda\right)^{1+\frac{2}{n}} \delta\left(x_{1}, \lambda\right) \theta_{0} \wedge d \theta_{0}^{n} \\
& \quad+\int_{B\left(x_{0}, \rho\right)} \frac{\delta\left(x_{1}, \lambda\right)}{\epsilon^{\frac{2}{n}} \lambda^{2} \lambda^{n}} \theta_{0} \wedge d \theta_{0}^{n} .
\end{aligned}
$$

Hence

$$
\begin{aligned}
& \frac{1}{\lambda^{n}} \int_{B\left(x_{0}, \rho\right)} \delta\left(x_{0}, \lambda\right)^{\frac{2}{n}} \delta\left(x_{1}, \lambda\right) \theta_{0} \wedge d \theta_{0}^{n} \\
& \leq \epsilon \int_{\mathbb{H}^{n}} \delta\left(x_{0}, \lambda\right)^{1+\frac{2}{n}} \delta\left(x_{1}, \lambda\right) \theta_{0} \wedge d \theta_{0}^{n} \\
& \quad+\frac{1}{\lambda^{2+n} \epsilon^{\frac{2}{n}}} \int_{B\left(x_{0}, \rho\right)} \delta\left(x_{1}, \lambda\right) \theta_{0} \wedge d \theta_{0}^{n}
\end{aligned}
$$

and

$$
\int_{B\left(x_{0}, \rho\right)} \delta\left(x_{1}, \lambda\right) \theta_{0} \wedge d \theta_{0}^{n} \leq \frac{C(\rho)}{\lambda^{\frac{n^{2}}{n+2}}}
$$


Thus

$$
\begin{aligned}
& \frac{1}{\lambda^{n}} \int_{B\left(x_{0}, \rho\right)} \delta\left(x_{0}, \lambda\right)^{\frac{2}{n}} \delta\left(x_{1}, \lambda\right) \theta_{0} \wedge d \theta_{0}^{n} \\
& \leq \epsilon \int_{\mathbb{H}^{n}} \delta\left(x_{0}, \lambda\right)^{1+\frac{2}{n}} \delta\left(x_{1}, \lambda\right) \theta_{0} \wedge d \theta_{0}^{n} \\
& \quad+\frac{C^{\prime}(\rho)}{\lambda^{2+n} \lambda^{\frac{n^{2}}{n+2}} \epsilon^{\frac{2}{n}}} .
\end{aligned}
$$

By using the inequality (v) of Lemma 4, we derive:

$$
\begin{aligned}
& \frac{1}{\lambda^{n}} \int_{B\left(x_{0}, \rho\right)} \delta\left(x_{0}, \lambda\right)^{\frac{2}{n}} \delta\left(x_{1}, \lambda\right) \theta_{0} \wedge d \theta_{0}^{n} \\
& \leq\left(\epsilon+\epsilon^{-\frac{2}{n}}\right) \int_{\mathbb{H}^{n}} \delta\left(x_{0}, \lambda\right)^{1+\frac{2}{n}} \delta\left(x_{1}, \lambda\right) \theta_{0} \wedge d \theta_{0}^{n} .
\end{aligned}
$$

Given $\eta>0$, we choose $\epsilon>0$ such that $\epsilon+\epsilon^{-\frac{2}{n}}=\eta$ and we derive for $\lambda$ large enough:

$$
\begin{aligned}
& \frac{1}{\lambda^{n}} \int_{B\left(x_{0}, \rho\right)} \delta\left(x_{0}, \lambda\right)^{\frac{2}{n}} \delta\left(x_{1}, \lambda\right) \theta_{0} \wedge d \theta_{0}^{n} \\
& \leq \eta \int_{\mathbb{H}^{n}} \delta\left(x_{0}, \lambda\right)^{1+\frac{2}{n}} \delta\left(x_{1}, \lambda\right) \theta_{0} \wedge d \theta_{0}^{n} .
\end{aligned}
$$

\section{Appendix B.}

We will prove the following result:

Lemma 27. If there is no solution of (1), then for $\epsilon>0$ small enough, $M$ is a retract of $J_{S+\epsilon}$.

First we note that if $\epsilon \rightarrow 0$, the functions $u$ of $J_{S+\epsilon}$ are in $V(1, \delta)$, where $\delta \rightarrow 0$ and $\epsilon \rightarrow 0$. We will give a short proof of this:

Let us suppose that there exists $u \in J_{S+\epsilon}$ such that $\left|J^{\prime}(u)\right| \geq \Theta>0$. Then, there is a ball of radius $\rho$ independent of $\epsilon$ such that $\left|J^{\prime}(w)\right| \geq \frac{\Theta}{2}$ on $B(u, \rho)$. The flow of the gradient of $J$ is defined by:

$$
\left\{\begin{array}{l}
\frac{\partial w}{\partial s}=-J^{\prime}(w) \\
w(0)=u
\end{array} .\right.
$$

The solution $w(s, u)$ exists on the ball $B(u, \rho)$, and we have:

$$
\frac{\partial}{\partial s} J(w(s, u))=-\left|J^{\prime}(w)\right|^{2} \leq-\frac{\Theta^{2}}{4} .
$$


$J$ is a decreasing function on the ball $B(u, \rho)$. Thus, the flow line must leave the ball, otherwise we will be below the level $S$. Let us suppose that the flow line leaves the ball at $s_{0}$. Then, we have:

$$
\rho \leq \int_{0}^{s_{0}}\left|\frac{\partial w}{\partial s}\right| d s=\int_{0}^{s_{0}}\left|J^{\prime}(w)\right| \leq c s_{0} .
$$

Thus, $s_{0} \geq \frac{\rho}{c}$ and

$$
\begin{aligned}
J\left(w\left(s_{0}, u\right)\right)=J(u)-\int_{0}^{s_{0}}\left|J^{\prime}(w)\right|^{2} d s & \leq S+\epsilon-\frac{\Theta^{2}}{4} s_{0} \\
& \leq S+\epsilon-\frac{\Theta^{2}}{4} \frac{\rho}{c}<S
\end{aligned}
$$

for $\epsilon$ small enough, which is a contradiction. Thus, $\Theta \rightarrow 0$ if $\epsilon \rightarrow 0$.

Proof of Lemma 26. Since $J_{S+\epsilon} \subset V(1, \delta)$ for $\epsilon$ small enough (then $\delta \rightarrow 0$ ) we can write for $u \in J_{S+\epsilon}, u=\alpha \hat{\delta}(a, \lambda)+v$ uniquely (see Lemma 7); then, we have the following correspondence:

$$
\begin{aligned}
M & \longrightarrow J_{S+\epsilon} \longrightarrow M \\
a & \longmapsto \hat{\delta}(a, \lambda) \longmapsto a
\end{aligned}
$$

with

$$
\begin{aligned}
J_{S+\epsilon} & \longrightarrow M \\
(u=\hat{\delta}(a, \lambda)+v) & \longmapsto a
\end{aligned}
$$

and

$$
\begin{aligned}
& M \longrightarrow J_{S+\epsilon} \\
& a \longmapsto \hat{\delta}(a, \lambda)
\end{aligned}
$$

(with $\lambda \longrightarrow+\infty$ ).

This establishes our claim. 


\section{Appendix C.}

In this appendix, we give an example in the case $M=S^{k}$ and $p=2$, where formula (6.7) is understood in an easier way.

The pair $\left(B_{2}\left(S^{k}\right), B_{1}\left(S^{k}\right)\right)$ can be viewed after excision (homologically) as:

$$
\left(\left(\left(S^{k}\right)^{2} \backslash T\right) \underset{\sigma_{2}}{\times} \Delta_{1}^{\eta}, \partial\left(\left(\left(S^{k}\right)^{2} \backslash T\right) \underset{\sigma_{2}}{\times} \Delta_{1}^{\eta}\right)\right)
$$

where $T$ is a tubular $\sigma_{2}$-equivariant neighborhood of the diagonal in $\left(S^{k}\right)^{2}$.

We have a natural covering of pairs with two sheets:

$$
\begin{aligned}
q:\left(\left(\left(S^{k}\right)^{2} \backslash T\right) \times \Delta_{1}^{\eta}, \partial\left(\left(\left(S^{k}\right)^{2} \backslash T\right) \times \Delta_{1}^{\eta}\right)\right) & \longrightarrow \\
& \left(\left(\left(S^{k}\right)^{2} \backslash T\right) \underset{\sigma_{2}}{\times} \Delta_{1}^{\eta}, \partial\left(\left(\left(S^{k}\right)^{2} \backslash T\right) \underset{\sigma_{2}}{\times} \Delta_{1}^{\eta}\right)\right)
\end{aligned}
$$

and there is a commutative diagram adapted to this covering:

$$
\begin{aligned}
& \left(\left(\left(S^{k}\right)^{2} \backslash T\right) \times \Delta_{1}^{\eta}, \partial\left(\left(\left(S^{k}\right)^{2} \backslash T\right) \times \Delta_{1}^{\eta}\right)\right) \longrightarrow S^{k} \\
& q \downarrow \\
& \downarrow \widetilde{q} \\
& \left(\left(\left(S^{k}\right)^{2} \backslash T\right) \underset{\sigma_{2}}{\times} \Delta_{1}^{\eta}, \partial\left(\left(\left(S^{k}\right)^{2} \backslash T\right) \underset{\sigma_{2}}{\times} \Delta_{1}^{\eta}\right)\right) \stackrel{\widetilde{s}}{\longrightarrow} \mathbb{P R}^{k}
\end{aligned}
$$

where $q$ associates to $\left(x, y, \alpha_{1}, \alpha_{2}\right) \in\left(\left(\left(S^{k}\right)^{2} \backslash T\right) \times \Delta_{1}^{\eta}, \partial\left(\left(\left(S^{k}\right)^{2} \backslash T\right) \times \Delta_{1}^{\eta}\right)\right)$ the point $\left[x, y, \alpha_{1}, \alpha_{2}\right] \in\left(\left(\left(S^{k}\right)^{2} \backslash T\right) \underset{\sigma_{2}}{\times} \Delta_{1}^{\eta}, \partial\left(\left(\left(S^{k}\right)^{2} \backslash T\right) \underset{\sigma_{2}}{\times} \Delta_{1}^{\eta}\right)\right)$, and $\widetilde{q}$ which is the natural projection map of $S^{k}$ in the projective space $\mathbb{P R}^{k}$, associates to $\frac{x-y}{|x-y|} \in S^{k}$ its class $\left[\frac{x-y}{|x-y|}\right]$ in $\mathbb{P R}^{k}$.

Let $\omega_{2}$, respectively $\widetilde{\omega}_{2}$ denote the orientation class of

$$
H_{*}\left(\left(\left(S^{k}\right)^{2} \backslash T\right) \underset{\sigma_{2}}{\times} \Delta_{1}^{\eta}, \partial\left(\left(\left(S^{k}\right)^{2} \backslash T\right) \underset{\sigma_{2}}{\times} \Delta_{1}^{\eta}\right)\right)
$$

respectively of

$$
H_{*}\left(\left(\left(S^{k}\right)^{2} \backslash T\right) \times \Delta_{1}^{\eta}, \partial\left(\left(\left(S^{k}\right)^{2} \backslash T\right) \times \Delta_{1}^{\eta}\right)\right) .
$$

We have:

$$
\operatorname{tr}_{*}\left(\omega_{2}\right)=\widetilde{\omega}_{2} .
$$

The orientation class $\widetilde{\omega}_{2}$, respectively $\omega_{2}$ can be viewed as the tensor product of the orientation class of $S^{k}$, respectively of $\mathbb{P R}^{k}$, with the orientation class of the fiber $\left(s^{-1}(\xi), \partial\left(s^{-1}(\xi)\right)\right)$, respectively $\left(\widetilde{s}^{-1}(\xi), \partial\left(\widetilde{s}^{-1}(\xi)\right)\right)$, where $\xi \in$ $S^{k}$, that is

$$
\begin{aligned}
\widetilde{\omega}_{2} & =\left[S^{k}\right] \otimes\left[s^{-1}(\xi), \partial\left(s^{-1}(\xi)\right)\right] \\
\omega_{2} & =\left[\mathbb{P} \mathbb{R}^{k}\right] \otimes\left[\widetilde{s}^{-1}[\xi], \partial\left(\widetilde{s}^{-1}[\xi]\right)\right] .
\end{aligned}
$$


We have by $\widetilde{q}$ :

$$
\operatorname{tr}^{*}\left(\left[S^{k}\right]\right)=\left[\mathbb{P R}^{k}\right] .
$$

Since we have $\widetilde{s} \circ q=\widetilde{q} \circ s$, then by naturality of the transfer (see [6], p. 121):

$$
\operatorname{tr}^{*} \circ s^{*}=\widetilde{s}^{*} \circ t r^{*}
$$

Then

$$
\operatorname{tr}^{*} \circ s^{*}\left(\left[S^{k}\right]\right)=\widetilde{s}^{*}\left(\left[\mathbb{P R}^{k}\right]\right)
$$

and if we take $u^{*}=s^{*}\left(\left[S^{k}\right]\right)$, and $\omega=\omega_{2}$ then the formula (6.7) becomes:

$$
q_{*}\left(s^{*}\left(\left[S^{k}\right]\right) \cap \operatorname{tr}_{*}\left(\omega_{2}\right)\right)=\operatorname{tr}^{*}\left(s^{*}\left(\left[S^{k}\right]\right)\right) \cap \omega_{2}
$$

that is:

$$
\begin{aligned}
& q_{*}\left(s^{*}\left(\left[S^{k}\right]\right) \cap\left(\left[S^{k}\right] \otimes\left[s^{-1}(\xi), \partial\left(s^{-1}(\xi)\right)\right]\right)\right) \\
& =\widetilde{s}^{*}\left(\left[\mathbb{P R}^{k}\right]\right) \cap\left(\left[\mathbb{P R}^{k}\right] \otimes\left[\widetilde{s}^{-1}([\xi]), \partial\left(\widetilde{s}^{-1}([\xi])\right)\right]\right) .
\end{aligned}
$$

In this way, the computation of the cap product is easy: We evaluate $\widetilde{s}^{*}\left(\left[\mathbb{P R}^{k}\right]\right)$ on $\left[\mathbb{P R}^{k}\right]$ and then, multiply by $\left[\widetilde{s}^{-1}([\xi]), \partial\left(\widetilde{s}^{-1}([\xi])\right)\right]$ on the right hand side of $(*)$. We evaluate $s^{*}\left(\left[S^{k}\right]\right)$ on $S^{k}$ and then multiply by $\left[s^{-1}(\xi), \partial\left(s^{-1}(\xi)\right)\right]$ on the left hand side of $(*)$.

The evaluation gives 1 on both sides, thus $(*)$ is equivalent to:

$$
q_{*}\left[s^{-1}(\xi), \partial\left(s^{-1}(\xi)\right)\right]=\left[\widetilde{s}^{-1}([\xi]), \partial\left(\widetilde{s}^{-1}([\xi])\right)\right] .
$$

Clearly by the commutativity of the diagram, we know that:

$$
q:\left(s^{-1}(\xi), \partial\left(s^{-1}(\xi)\right)\right) \longmapsto\left(\widetilde{s}^{-1}([\xi]), \partial\left(\widetilde{s}^{-1}([\xi])\right)\right)
$$

is a diffeomorphism, and $\widetilde{s}$ is $s$ after taking the quotient by the action of $\sigma_{2}$. We then have an isomorphism between $s^{-1}(\xi)$ and $\widetilde{s}^{-1}([\xi])$. Thus:

$$
q_{*}: H_{*}\left(s^{-1}(\xi), \partial\left(s^{-1}(\xi)\right)\right) \longrightarrow H_{*}\left(\widetilde{s}^{-1}([\xi]), \partial\left(\widetilde{s}^{-1}([\xi])\right)\right)
$$

is an isomorphism and this gives the result.

Rigorously speaking, the homological class used in the proof of Lemma 25, which we denote $u^{*}$, is not obtained through the covering defined by $s$ but rather by $p_{1}$ where:

$$
\begin{aligned}
p_{1}:\left(\left(S^{k}\right)^{2} \backslash T\right) \times \Delta_{1}^{\eta} & \longrightarrow S^{k} \\
\left(x, y, \alpha_{1}, \alpha_{2}\right) & \longmapsto x .
\end{aligned}
$$

$p_{1}$ cannot be inserted in a commutative diagram such as the one used for $s$, but the following statement shows that $p_{1}$ and $s$ can be related homotopically, and then all what we did for $s$ holds for the map $p_{1}$ : 
We consider the function

$$
\begin{aligned}
r:[0,1] \times\left(\left(\left(S^{k}\right)^{2} \backslash T\right) \times \Delta_{1}\right) & \longrightarrow S^{k} \\
\left(t ; x, y, \alpha_{1}, \alpha_{2}\right) & \longmapsto \frac{x-t y}{|x-t y|} .
\end{aligned}
$$

We have $r(0,)=.p_{1}$ and $r(1,)=$.$s . Thus, p_{1 *}=s_{*}$ and $p_{1}^{*}=s^{*}$. Thus:

$$
u^{*}=s^{*}\left(\left[S^{k}\right]\right)=p_{1}^{*}\left(\left[S^{k}\right]\right)
$$

and the formula

$$
\operatorname{tr}^{*}\left(p_{1}^{*}\left(\left[S^{k}\right]\right)\right) \cap \omega_{2}=q_{*}\left(p_{1}^{*}\left(\left[S^{k}\right]\right) \cap t r_{*} \omega_{2}\right)
$$

is identical to

$$
\operatorname{tr}^{*}\left(s^{*}\left(\left[S^{k}\right]\right)\right) \cap \omega_{2}=q_{*}\left(s^{*}\left(\left[S^{k}\right]\right) \cap t r_{*}\left(\omega_{2}\right)\right)
$$

which we have established.

\section{References}

[1] T. Aubin, Equations differentielles non linéaires et problème de Yamabe concernant la courbure scalaire, J. Math. Pures et Appl., 55 (1976), 269-296, MR 55 \#4288, Zbl 336.53033.

[2] A. Bahri, Proof of the Yamabe conjecture for locally conformally flat manifolds, Non. Analysis, Theory, Methods and Appli., 20(10) (1993), 1261-1278, MR 94e:53033, Zbl 782.53027.

[3] _ Critical points at infinity in some variational problems, Pitman Research Notes in Mathematics Series, 182 (Longman), 1989, MR 91h:58022, Zbl 676.58021.

[4] A. Bahri and H. Brezis, Nonlinear elliptic equations, in 'Topics in Geometry in memory of Joseph D'Atri', Simon Gindikin editor, Birkhäuser, Boston-Basel-Berlin, (1996), 1-100, Zbl 863.35037.

[5] A. Bahri and J.M. Coron, On a non linear elliptic equation involving the critical Sobolev exponent, the effect of the topology of the domain, Commun. on Pure \& Appl. Math., XLI (1988), 253-294, MR 89c:35053, Zbl 649.35033.

[6] G.E. Bredon, Topology and Geometry, Graduate Texts in Mathematics, 139, Springer Verlag, 1997, MR 2000b:55001, Zbl 934.55001.

[7] A. Dold, Lectures on Algebraic Topology, A series of comprehensive studies in Mathematics, Springer Verlag, 200 (1972), MR 54 \#3685, Zbl 234.55001.

[8] G.B. Folland and E. Stein, Estimates for the $\bar{\partial}_{b}$ complex and analysis on the Heisenberg group, Comm. Pures Appl. Math., 27 (1974), 429-522, MR 51 \#3719, Zbl 293.35012.

[9] N. Gamara, CR Yamabe conjecture, the case $n=1$, J. Eur. Math. Soc. 3 (2001), 105-137.

[10] D. Jerison and J.M. Lee, The Yamabe problem on CR manifolds, J. Diff. Geom., 25 (1987), 167-197, MR 88i:58162, Zbl 661.32026. 
[11] , Intrinsic CR normal coordinates and the CR Yamabe problem, J. Diff. Geom., 29 (1989), 303-343, MR 90h:58083, Zbl 671.32016.

[12] Extremals for the Sobolev inequality on the Heisenberg group and the CR Yamabe problem, J. Amer. Math. Soc., 1(1) (1988), 1-13 (H. Jacobowitz), MR 89b:53063, Zbl 634.32016.

[13] J.J. Kohn, Boundaries of complex manifolds, in 'Proc. Conf. on Complex Analysis' (Minneapolis, 1964), Springer Verlag, Berlin, (1965), 81-94, MR 30 \#5334, Zbl 166.36003.

[14] J.M. Lee, The Fefferman metric and pseudohermitian invariants, Trans. Amer. Math. Soc., 296 (1986), 411-429, Zbl 595.32026.

[15] Z. Li, Webster scalar curvature problem on CR manifolds, preprint.

[16] G. Lu, Existence and size estimates for the Green's functions of differential operators constructed from degenerate vector fields, Comm. In Partial Diff. Equations, 17 (1992), 1213-1251, MR 93i:35030, Zbl 798.35002.

[17] G. Lu, Weighted Poincaré and Sobolev inequalities for vector fields satisfying Hörmander's conditions and applications, Revista Matimatica Iberoamericana, 8(3) (1992), 367-439, MR 94c:35061, Zbl 804.35015.

[18] R. Schoen, Conformal deformation of a metric to constant scalar curvature, J. Diff. Geom., 20 (1984), 479-495, MR 86i:58137, Zbl 576.53028.

[19] M. Struwe, A Global Compactness Result for Elliptic Boundary Value Problems Involving Limiting Nonlinearities, Mathematische Zeitschrift, 187, Springer Verlag (1984), 511-517, MR 86k:35046, Zbl 545.35034.

Received November 8, 1999 and revised April 3, 2000.

Faculté des Sciences de Tunis

DÉPARTEMENT DE MATHÉMATIQUES

CAmpus Universitaire

LE BELVEDERE 1060

TuNis

TUNISIE

E-mail address: Najoua.Gamara@fst.rnu.tn

Institut Préparatoire aux Etudes D'Ingénieurs de Monastir

Route De Kairouan 5019

MONASTIR

TUNisie

E-mail address: ridha.yacoub@ipeim.rnu.tn 